\title{
Single crystal processing and magnetic properties of GdNi
}

$$
\text { by }
$$

\section{Andrew John Sherve}

\author{
A thesis submitted to the graduate faculty \\ in partial fulfillment of the requirements for the degree of \\ MASTER OF SCIENCE
}
Major: Materials Science and Engineering
Program of Study Committee:
Karl A. Gschneidner Jr., Co-major Professor
Vitalij K. Pecharsky, Co-major Professor
Gordon J. Miller

\author{
Iowa State University \\ Ames, Iowa
}

2012

Copyright (C) Andrew John Sherve, 2012. All rights reserved. 
UMI Number: 1512353

All rights reserved

INFORMATION TO ALL USERS

The quality of this reproduction is dependent on the quality of the copy submitted.

In the unlikely event that the author did not send a complete manuscript and there are missing pages, these will be noted. Also, if material had to be removed, a note will indicate the deletion.

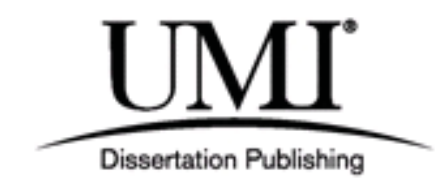

UMI 1512353

Copyright 2012 by ProQuest LLC.

All rights reserved. This edition of the work is protected against unauthorized copying under Title 17, United States Code.

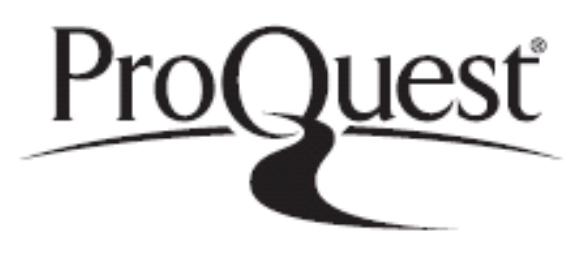

ProQuest LLC.

789 East Eisenhower Parkway

P.O. Box 1346

Ann Arbor, MI 48106 - 1346 


\section{Table of Contents}

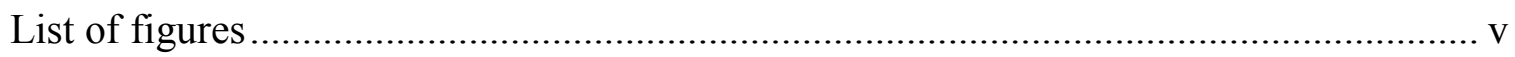

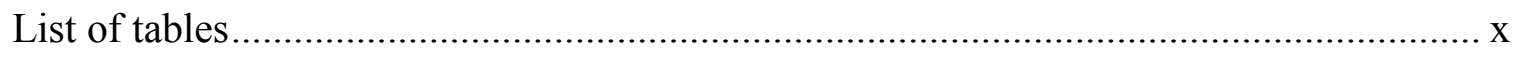

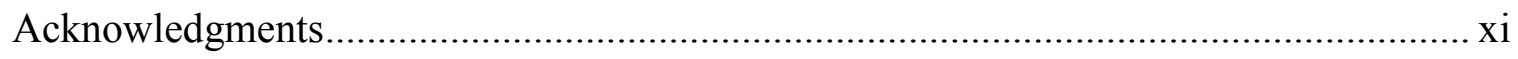

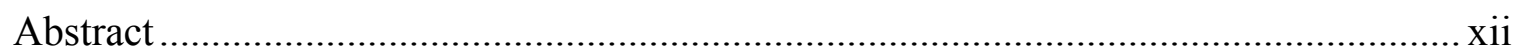

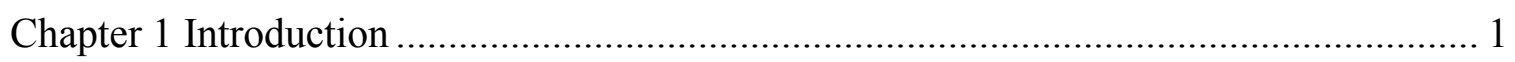

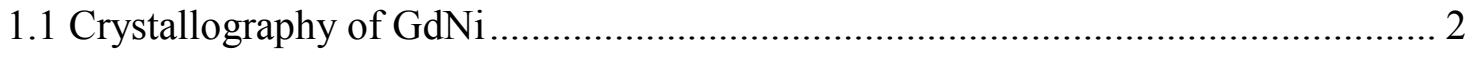

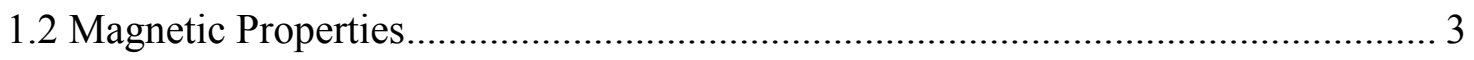

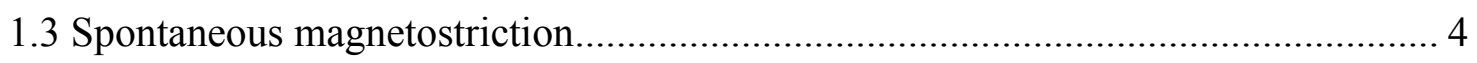

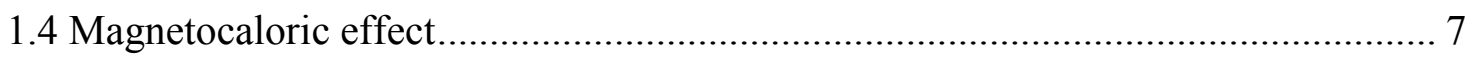

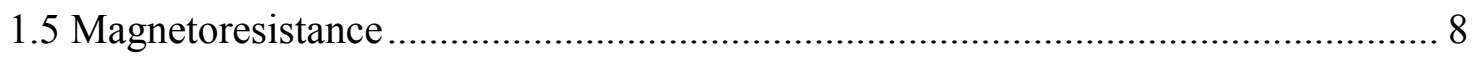

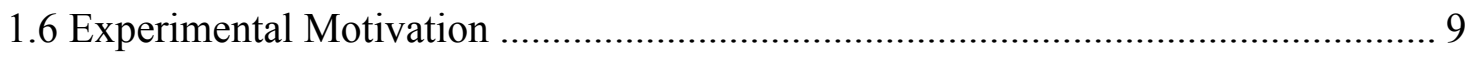

Chapter 2 Experimental techniques .......................................................................... 12

2.1 Materials sources and sample preparation ..................................................... 12

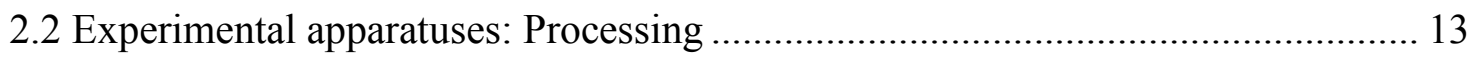

2.2.1 Tri-arc crystal pulling method .................................................................... 13

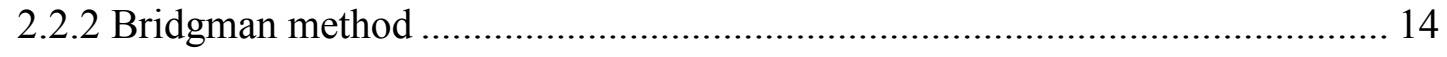

2.3 Experimental apparatuses: Characterization ....................................................... 14

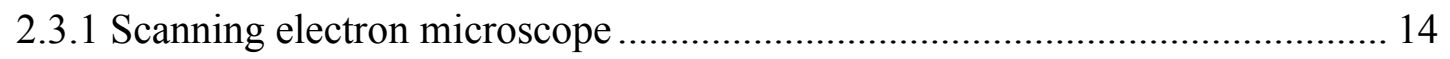


2.3.2 Laue backscatter diffraction ..................................................................... 14

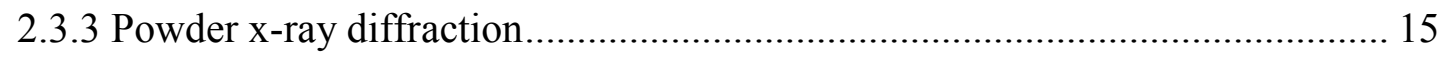

2.3.4 Magnetic property measurement system ………………............................... 15

2.3.5 Physical property measurement system........................................................ 15

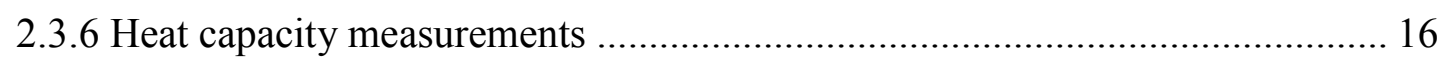

Chapter 3 Single crystal growth attempts by tri-arc crystal pulling method ................... 17

Chapter 4 Precipitation growth of GdNi.............................................................. 19

Chapter 5 GdNi Processing via commercially available crucibles .................................. 23

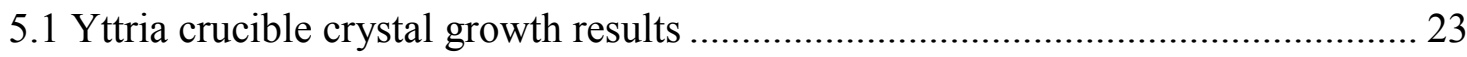

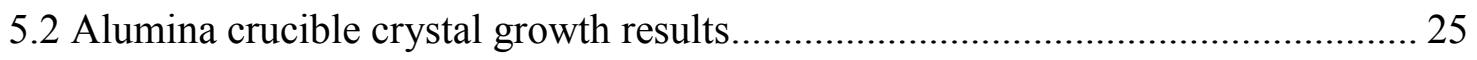

Chapter 6 GdNi Processing via coated Ta crucibles results ............................................ 26

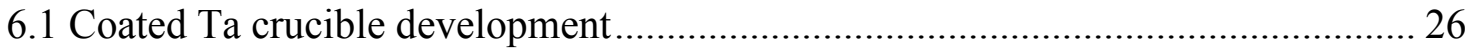

6.2 Coated Ta crucible crystal growth results ............................................................ 30

Chapter 7 GdNi Processing via plasma-sprayed powder crucibles ................................. 35

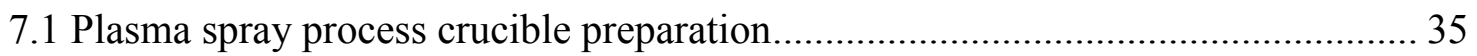

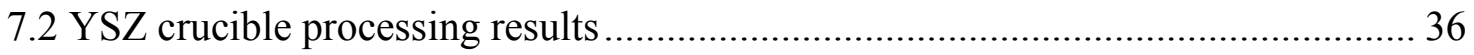

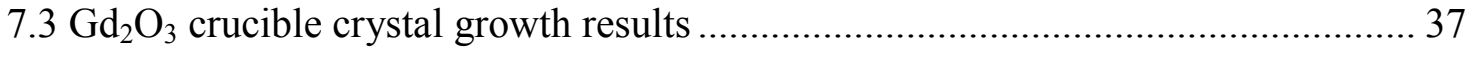

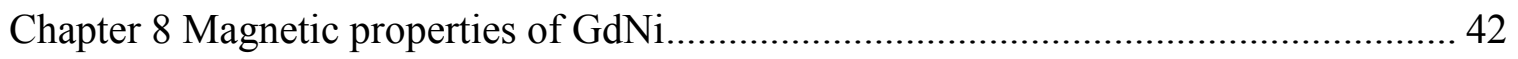

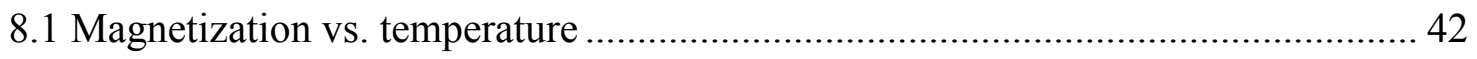




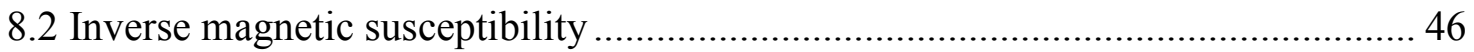

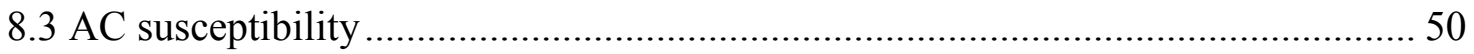

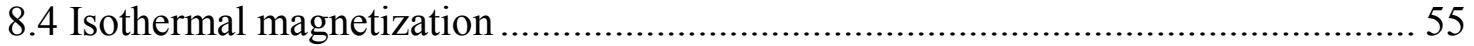

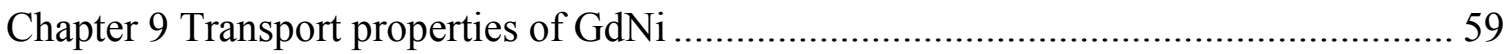

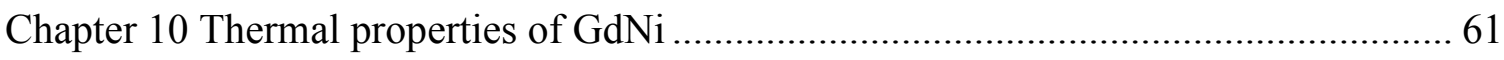

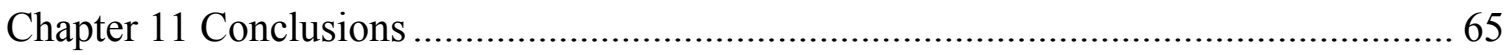

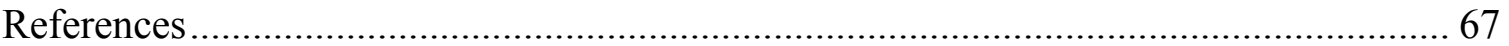




\section{List of figures}

Figure 1.1: The unit cell of $\mathrm{GdNi}$, a $\mathrm{CrB}(\mathrm{Cmcm})$ type structure (taken from Ref. 20) ..... 3

Figure 1.2: The spontaneous magnetostriction of GdNi. The lattice parameters are

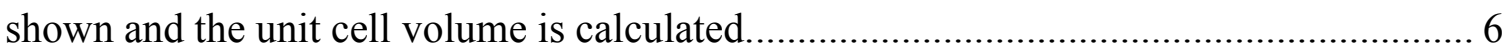

Figure 1.3: The magnetostrictive effect in GdNi shown in ppm strain. ........................... 6

Figure 1.4: The progression of crystal structures of the RNi system............................... 11

Figure 3.1: Three GdNi samples drawn in a tri-arc in the crystal pulling method. .......... 18

Figure 4.1: The Gd-Ni phase diagram (taken from Ref. 32), ........................................... 20

Figure 4.2: The $\mathrm{M}(\mathrm{T})$ curve of a flux-grown GdNi single crystal along the a direction

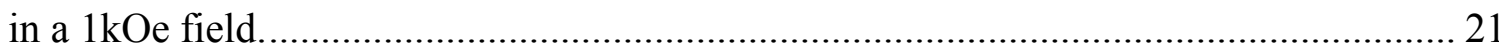

Figure 4.3: An SEM image viewing a flux grown GdNi single crystal.......................... 22

Figure 5.1: The GdNi melt and interaction zone interface........................................... 24

Figure 5.2: The interaction zone and $\mathrm{Y}_{2} \mathrm{O}_{3}$ interface................................................. 24

Figure 5.3: An image of YNi grown in an alumina crucible......................................... 25

Figure 6.1: The bottom of a tantalum carbide coated tantalum crucible with GdNi

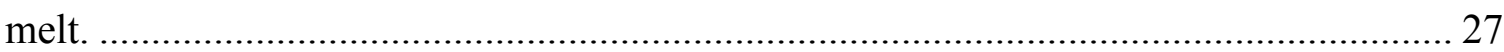

Figure 6.2: The wall of a tantalum boride coated tantalum crucible with GdNi ............ 28

Figure 6.3: GdNi single crystal growth in tantalum boride coated tantalum, scale bar -

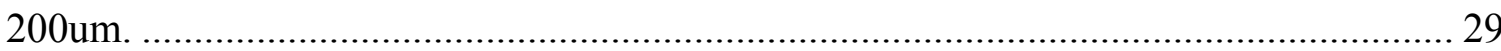

Figure 6.4: A cross-section of a GdNi crystal growth in a tantalum boride coated tantalum crucible

Figure 6.5: A polished single crystal of GdNi grown in tantalum boride coated tantalum in SEM. 
Figure 6.6: A cross-section SEM image of a tantalum boride coated tantalum crucible and GdNi crystal growth near the meniscus. 32

Figure 6.7: The Rietveld refinement and fit of GdNi powder from a single crystal grown in a tantalum boride coated tantalum crucible. No impurity phases are seen....... 34

Figure 7.1: A cross-section SEM image of GdNi in a YSZ test cruible......................... 37

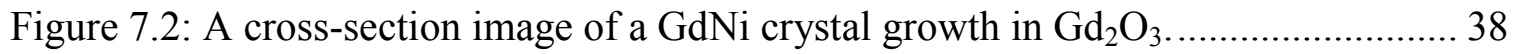

Figure 7.3: SEM image of a polished single crystal of $\mathrm{GdNi}$ grown in $\mathrm{Gd}_{2} \mathrm{O}_{3}$.............. 39

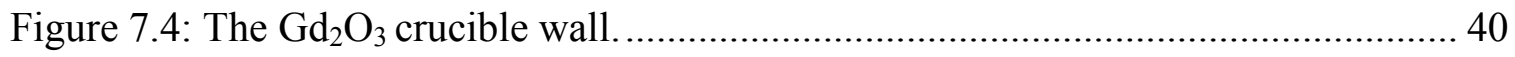

Figure 7.5: $\mathrm{A} \mathrm{Gd}_{2} \mathrm{O}_{3}$ crucible fragment from the meniscus region indicating some of

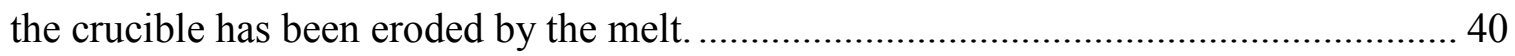

Figure 7.6: The Rietveld refinement and fit of GdNi powder from a single crystal grown in a $\mathrm{Gd}_{2} \mathrm{O}_{3}$ crucible. No impurity phases are seen.

Figure 8.1: The ZFC M(T) curves in $1 \mathrm{kOe}$ and $100 \mathrm{Oe}$ fields for single crystal GdNi, $\mathrm{H} \|$.

Figure 8.2: The ZFC M(T) curves in $1 \mathrm{kOe}, 20 \mathrm{kOe}$ and $40 \mathrm{kOe}$ fields for single crystal GdNi, $\mathrm{H}|| a$

Figure 8.3: The ZFC M(T) curves in $1 \mathrm{kOe}$ and $100 \mathrm{Oe}$ fields for single crystal GdNi, $\mathrm{H}|| b$.

Figure 8.4: The ZFC M(T) curves in $1 \mathrm{kOe}, 20 \mathrm{kOe}$ and $40 \mathrm{kOe}$ fields for single crystal GdNi, $\mathrm{H}|| b$

Figure 8.5: The ZFC M(T) curves in $1 \mathrm{kOe}$ and $100 \mathrm{Oe}$ fields for single crystal GdNi, $\mathrm{H} \| c$. 
Figure 8.6: The ZFC M(T) curves in $1 \mathrm{kOe}, 20 \mathrm{kOe}$ and 40kOe fields for single crystal GdNi, $\mathrm{H} \| c$ 46

Figure 8.7: Inverse magnetic susceptibility for single crystal GdNi grown in tantalum boride coated tantalum, $\mathrm{H} \| a$.

Figure 8.8: Inverse magnetic susceptibility for single crystal GdNi grown in $\mathrm{Gd}_{2} \mathrm{O}_{3}$,

$\mathrm{H} \| a$.

Figure 8.9: Inverse magnetic susceptibility for single crystal GdNi grown in tantalum boride coated tantalum, $\mathrm{H} \| b$.

Figure 8.10: Inverse magnetic susceptibility for single crystal GdNi grown in $\mathrm{Gd}_{2} \mathrm{O}_{3}$, $\mathrm{H} \| b$.

Figure 8.11: Inverse magnetic susceptibility for single crystal GdNi grown in tantalum boride coated tantalum, $\mathrm{H} \| c$.

Figure 8.12: Inverse magnetic susceptibility for single crystal GdNi grown in $\mathrm{Gd}_{2} \mathrm{O}_{3}$, $\mathrm{H} \| c$

Figure 8.13: Real component of AC susceptibility measured along the $a$-axis of single crystal GdNi grown in $\mathrm{Gd}_{2} \mathrm{O}_{3}$.

Figure 8.14: Imaginary component of AC susceptibility measured along the $a$-axis of single crystal GdNi grown in $\mathrm{Gd}_{2} \mathrm{O}_{3}$.

Figure 8.15: Real component of AC susceptibility measured along the $b$-axis of single crystal GdNi grown in $\mathrm{Gd}_{2} \mathrm{O}_{3}$

Figure 8.16: Imaginary component of AC susceptibility measured along the $b$-axis of single crystal GdNi grown in $\mathrm{Gd}_{2} \mathrm{O}_{3}$. 
Figure 8.17: Real component of AC susceptibility measured along the $c$-axis of single

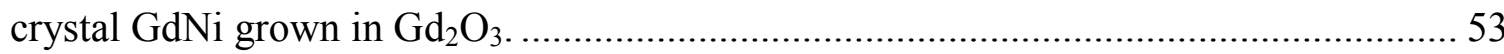

Figure 8.18: Imaginary component of AC susceptibility measured along the $c$-axis of

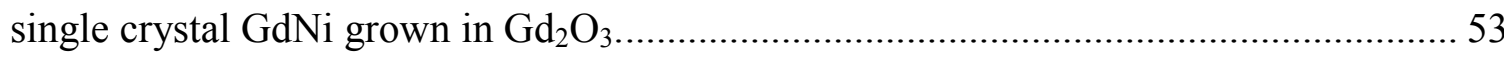

Figure 8.19: Real component of AC susceptibility measured along the $b$-axis of single crystal GdNi grown in tantalum boride coated tantalum.

Figure 8.20: Imaginary component of AC susceptibility measured along the $b$-axis of single crystal GdNi grown in tantalum boride coated tantalum.

Figure 8.21: The isothermal magnetization of single crystal GdNi along $a, b$, and

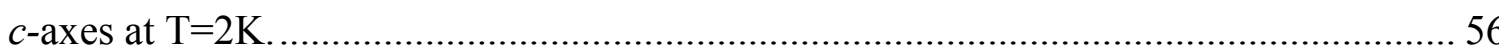

Figure 8.22: The isothermal magnetizations of single crystal $\mathrm{GdNi}$ from $\mathrm{T}=5 \mathrm{~K}$ to

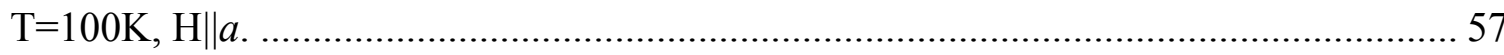

Figure 8.23: The isothermal magnetizations of single crystal $\mathrm{GdNi}$ from $\mathrm{T}=5 \mathrm{~K}$ to $\mathrm{T}=100 \mathrm{~K}, \mathrm{H} \| b$ 57

Figure 8.24: The isothermal magnetizations of single crystal $\mathrm{GdNi}$ from $\mathrm{T}=5 \mathrm{~K}$ to $\mathrm{T}=100 \mathrm{~K}, \mathrm{H} \| c$ 58

Figure 9.1: The resistivity vs. temperature curves for single crystal GdNi in zero field and $10 \mathrm{kOe}$ field, current flows parallel to the $a$-axis 59 Figure 9.2: The magnetoresistance of single crystal GdNi, in a 10kOe field, current along the $a$-axis.

Figure 10.1: The heat capacity of a GdNi single crystal measured on the b-axis (easy magnetization direction) measured in zero field, and 10, 20, and $50 \mathrm{kOe}$ fields 61

Figure 10.2: The temperature dependent entropy of single crystal GdNi 62 
Figure 10.3: Adiabatic temperature change as a function of temperature in single crystal GdNi along the $b$-axis, calculated from temperature change due to a magnetic

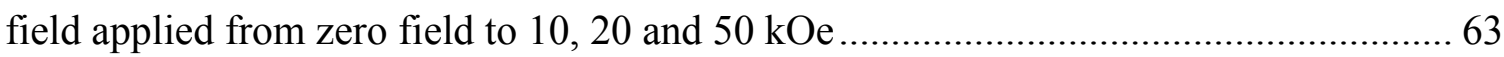

Figure 10.4: Isothermal magnetic entropy change plotted vs. temperature in single crystal GdNi along the $b$-axis, calculated from the entropy change due to a magnetic field applied from zero field to 10,20 and $50 \mathrm{kOe}$. 


\section{List of tables}

Table 6.1: A historical representation of the lattice parameters of GdNi at room

temperature

Table 8.1: The Weiss temperatures and effective moments calculated from the 40kOe

$\mathrm{H} / \mathrm{M}(\mathrm{T})$ data from the $\mathrm{Gd}_{2} \mathrm{O}_{3}$ grown single crystal.

Table 10.1: $\Delta \mathrm{T}_{\mathrm{ad}}{ }^{\max },-\Delta \mathrm{S}_{\mathrm{M}}{ }^{\max }$ and $\mathrm{RCP}$ values calculated from the heat capacity

measurements of GdNi 


\section{Acknowledgments}

I would like thank Drs. Karl Gschneidner, Vitalij Pecharsky, and Thomas Lograsso for

guiding me in my research and providing me with such an exciting research opportunity, and I would also like to thank Dr. Gordon Miller for serving on my graduate committee and Drs. Iver Anderson and Bill McCallum for contributing extraordinarily helpful advice at critical times.

I owe a debt of gratitude to the staff of the Ames Laboratory Materials Preparation Center and other Ames Laboratory staff for their endless knowledge, resources and helpfulness. I would like to specifically thank Yaroslav Mudryk, Deborah Schalgel, Rick Schmidt, Roger Rink, Hal Sailsbury, Dave Byrd for their close work making this project possible.

I would also like to acknowledge the loving support of my family and friends. Most importantly I would like to thank my girlfriend Stephanie for her love, and patience during this venture.

This work was supported by the Office of Basic Energy Sciences, Materials Sciences Division of the US Department of Energy under Contract No. DE-AC02-07CH11358 with Iowa State University. 


\section{Abstract}

GdNi is a rare earth intermetallic material that exhibits very interesting magnetic properties. Spontaneous magnetostriction occurs in GdNi at $\mathrm{T}_{\mathrm{C}}$, on the order of $8000 \mathrm{ppm}$ strain along the $c$-axis and only until very recently the mechanism causing this giant magnetostriction was not understood. In order to learn more about the electronic and magnetic structure of $\mathrm{GdNi}$, single crystals are required for anisotropic magnetic property measurements. Single crystal processing is quite challenging for GdNi though since the rare-earth transition-metal composition yields a very reactive intermetallic compound. Many crystal growth methods are pursued in this study including crucible free methods, precipitation growths, and specially developed Bridgman crucibles. A plasma-sprayed $\mathrm{Gd}_{2} \mathrm{O}_{3} \mathrm{~W}$-backed Bridgman crucible was found to be the best means of GdNi single crystal processing. With a source of high-quality single crystals, many magnetization measurements were collected to reveal the magnetic structure of GdNi. Heat capacity and the magnetocaloric effect are also measured on a single crystal sample. The result is a thorough report on high quality single crystal processing and the magnetic properties of GdNi. 


\section{Chapter 1 Introduction}

The GdNi compound was first alloyed and characterized in 1961 by two groups, Novy et al. mapping the gadolinium-nickel phase diagram ${ }^{1}$ and Baenziger et al. reporting the crystal structures of intermetallic compounds. ${ }^{2}$ Unfortunately these two publications were not in agreement over the crystal structure of GdNi. In 1964 two more publications from Walline et al. and Abrahams et al. would conclude that the crystal structure of GdNi is the $\mathrm{CrB}(\mathrm{Cmcm})$ crystal structure type. ${ }^{3,4}$ Beginning with these two papers, and following over the next three decades, the basic magnetic, electrical, and thermal properties of GdNi were established ${ }^{5-14}$. GdNi was discovered to have spontaneous magnetostriction properties in 1998 by Gratz et al. finding giant magnetostriction when cooling below the Curie temperature without a crystal structure transformation. ${ }^{15}$ Uhlirova et al., 2006, followed up on these findings with a single crystal study of the magnetostrictive behavior. ${ }^{16}$ Paudyal et al., 2008, confirmed the experimental findings and explained the mechanism behind the magnetostriction as changes in the electronic structure due to ferromagnetism, specifically Gd $5 \mathrm{~d}$ and $\mathrm{Ni} 3 \mathrm{~d}$ band hybridization that induces shifts in the free energy minima of the crystal lattice. ${ }^{17}$ Baranov et al., closely followed by Kumar et al. both measured a lambda-type (second order) ferromagnetic-to-paramagnetic transition in GdNi, ${ }^{18,19}$ confirmed by Paudyal et al. ${ }^{17}$ Kumar et al. found the magnetocaloric effect to be comparable to other materials with transitions in the same temperature range. $^{19}$ 


\subsection{Crystallography of GdNi}

The intermetallic compound $\mathrm{GdNi}$ is of the orthorhombic $\mathrm{Cmcm}$ space group $(\mathrm{CrB})$ with room temperature lattice parameters $a=3.778, b=10.365, c=4.221 \AA .{ }^{10}$ In Figure 1.1, an illustration of the GdNi structure is provided. ${ }^{20}$ Early reports of GdNi claimed the FeB (Pnma) structure $^{2}$ was observed but later attempts to confirm the phase were not successful. ${ }^{4} \mathrm{GdNi}$ remains in the $\mathrm{CrB}$ structure throughout the temperature range as a solid, even though there is large spontaneous magnetostriction related to ferromagnetic ordering. The orthorhombic structure gives GdNi anisotropic properties for most characterizations. The structure has "layers" or "chains" of Gd or Ni atoms layered in the $b$ plane. When stressed, either externally or internally, such as during solidification and cooling, cleavage along the $b$ plane is common. 


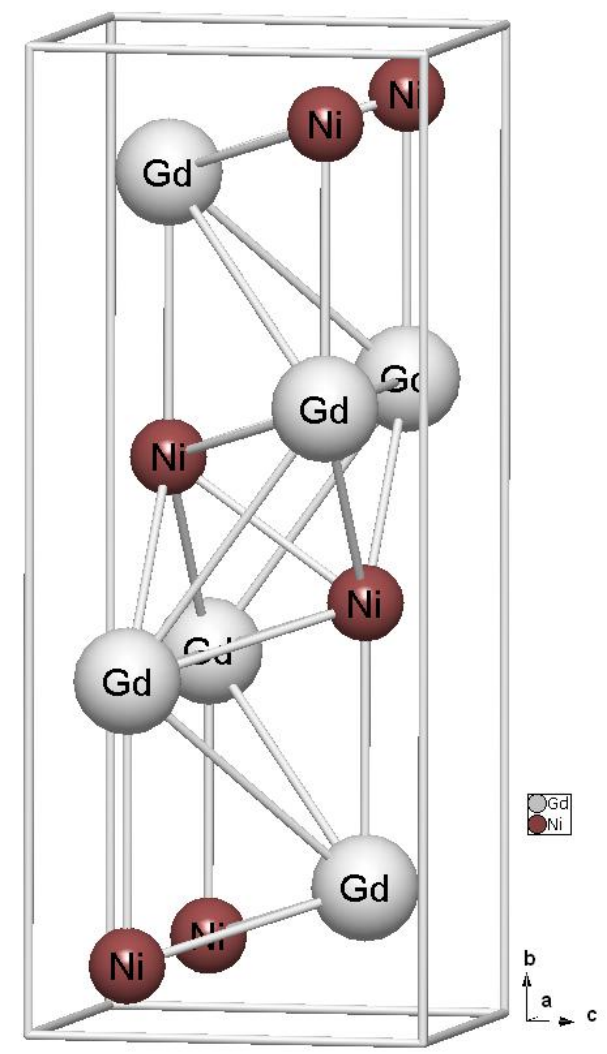

Figure 1.1: The unit cell of $\mathrm{GdNi}$, a $\mathrm{CrB}(\mathrm{Cmcm})$ type structure (taken from Ref. 20)

\subsection{Magnetic Properties}

GdNi is ferromagnetic (FM) below $69 \mathrm{~K}$ and paramagnetic (PM) above. Early measurements reported the Curie temperature $\left(\mathrm{T}_{\mathrm{C}}\right)$ around $71-73 \mathrm{~K},{ }^{3,4}$ but as constituent material purities and experimental techniques improved, the Curie temperature has settled at $69 \mathrm{~K} .^{11,12,16,17,19}$ The Weiss temperature $\left(\theta_{\mathrm{P}}\right)$ is $72.8 \mathrm{~K}$ and the effective magnetic moment ( $p_{\text {eff }}$ ) is $8.23 \mu_{\mathrm{B}} /$ f.u. calculated from the Curie-Weiss law. ${ }^{17}$ The theoretical value of $\mathrm{p}_{\text {eff }}$ from non-interacting magnetic ions is $7.94 \mu_{\mathrm{B}} / \mathrm{Gd}^{3+}$ ion calculated from the quantum numbers of Gd.

$$
p_{\text {eff }}=g \sqrt{J(J+1)}
$$


The Lande $g$-factor is $g$ and the atomic angular momentum is $J$. For Gd, $J=7 / 2$ and $g=2$. The effective magnetic moment is frequently measured to be larger than the theoretical value because of contributions from the conduction electrons of Gd. The saturated magnetic moment of the $\mathrm{Gd}^{3+}$ ion, $\mu$, is theoretically $7 \mu_{\mathrm{B}}$ from the combination of the Lande g-factor $g$ and the atomic angular momentum $J$.

$$
\mu=g J
$$

GdNi was previously measured to be $7.2 \mu_{\mathrm{B}} /$ f.u. ${ }^{16}$ Paudyal et al. calculated a saturation moment of $7.20 \mu_{\mathrm{B}} /$ f.u. from first principles to be a sum of moments from Gd $4 f$ of $7.00 \mu_{\mathrm{B}}, \mathrm{Gd} 5 d$ of $0.30 \mu_{\mathrm{B}}$, and Ni $3 d$ of $-0.10 \mu_{\mathrm{B}}{ }^{17,21}$ The antiferromagnetic alignment of the Ni moment was also experimentally measured by Yano et al. ${ }^{14}$ The easy magnetic direction is along the $b$-axis, reaching saturation in a $2 \mathrm{kOe}$ field, followed by the $a$-axis at $5 \mathrm{kOe}$ and the $c$-axis is the hard direction at $9 \mathrm{kOe}$ for the single crystal grown by Uhlirova et al. ${ }^{16}$

\subsection{Spontaneous magnetostriction}

The magnetostrictive property, as observed in GdNi is a spontaneous change in lattice parameters induced by a temperature and/or magnetic field change related to magnetic ordering. The magnitude of lattice parameter change is on the order of $8000 \mathrm{ppm}$ strain which is considered giant magnetostriction. The property was discovered in 1998 in $\mathrm{RNi}$ $\left(\mathrm{R}=\right.$ rare earth) compounds by Gratz and Lindbaum ${ }^{15}$ and the same group of researchers reported similar effects in $\mathrm{RCu}_{2}$ and $\mathrm{RCo}_{2}$ compounds earlier. ${ }^{22} \mathrm{~A}$ unique attribute of this property in GdNi is the nearly zero unit cell volume change throughout this transition. The transition initializes near the Curie temperature and concludes around $10 \mathrm{~K}$ in zero 
magnetic field. Applying a magnetic field was found to induce magnetostriction at higher temperatures, around $90-95 \mathrm{~K}$ in a $40 \mathrm{kOe}$ field. The temperature dependent lattice effects of spontaneous magnetostriction can be seen in Figure 1.2. Within approximately $10 \mathrm{~K}$ of $\mathrm{T}_{\mathrm{C}}$, GdNi magnetostriction can be induced by a magnetic field, and the dimensional change can be induced variably with the strength of the magnetic field. ${ }^{17}$ Figure 1.3 shows the magnetostrictive effect demonstrated on a GdNi powder sample. ${ }^{17}$ Magnetostriction in GdNi is particularly unique compared to other common magnetostrictive materials. Paudyal et al. found from a theoretical study of the electronic structure of GdNi that the Gd $5 d$ and Ni $3 d$ bands hybridize strongly during magnetic ordering causing anisotropic dimensional changes to the unit cell to minimize total free energy. ${ }^{17}$ Joule magnetostriction is the original definition of magnetostriction, and the mechanism of strain is domain rotation. This property was first discovered in $\mathrm{Fe}$ and $\mathrm{Ni}$ by Joule. ${ }^{23}$ A more recent class of materials have been studied where the mechanism for magnetostriction is a change in the crystal structure such as Gd-Si-Ge compounds. ${ }^{24-26}$ The mechanism of spontaneous magnetostriction in GdNi is different from these more common definitions of magnetostriction and is therefore, quite an intriguing material. 


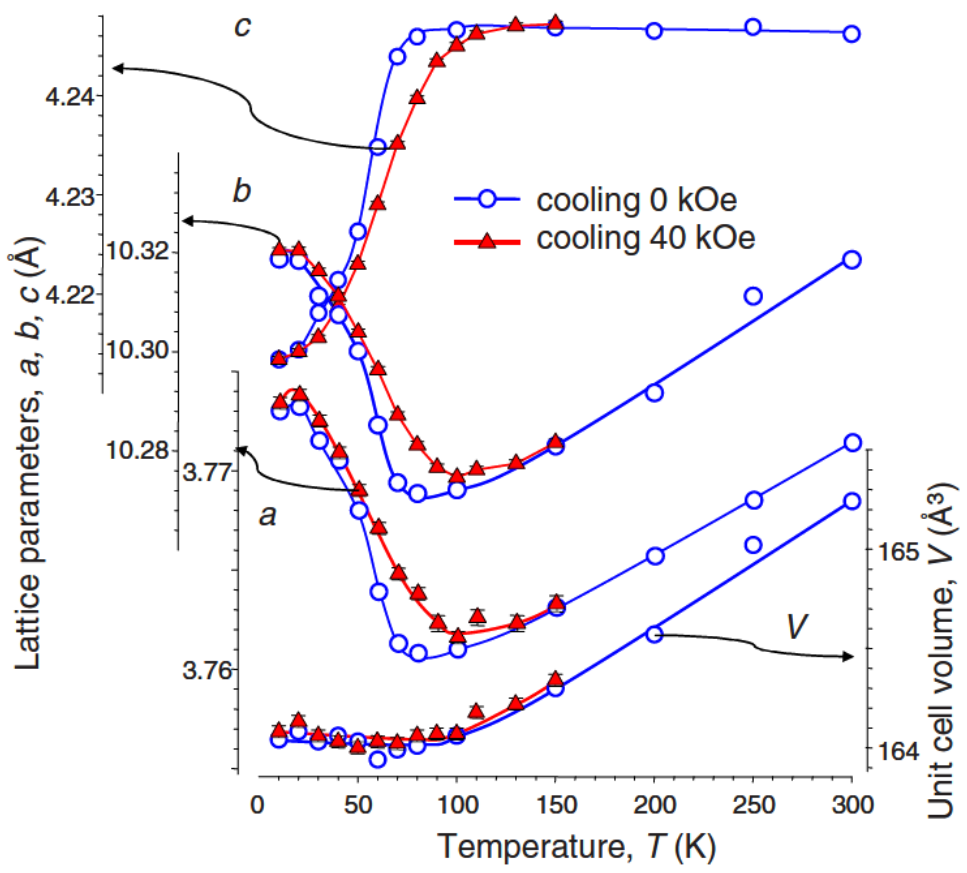

Figure 1.2: The spontaneous magnetostriction of GdNi. The lattice parameters are shown and the unit cell volume is calculated. Lattice parameters in zero field are in blue, $40 \mathrm{kOe}$ field in red (taken from Ref. 17).

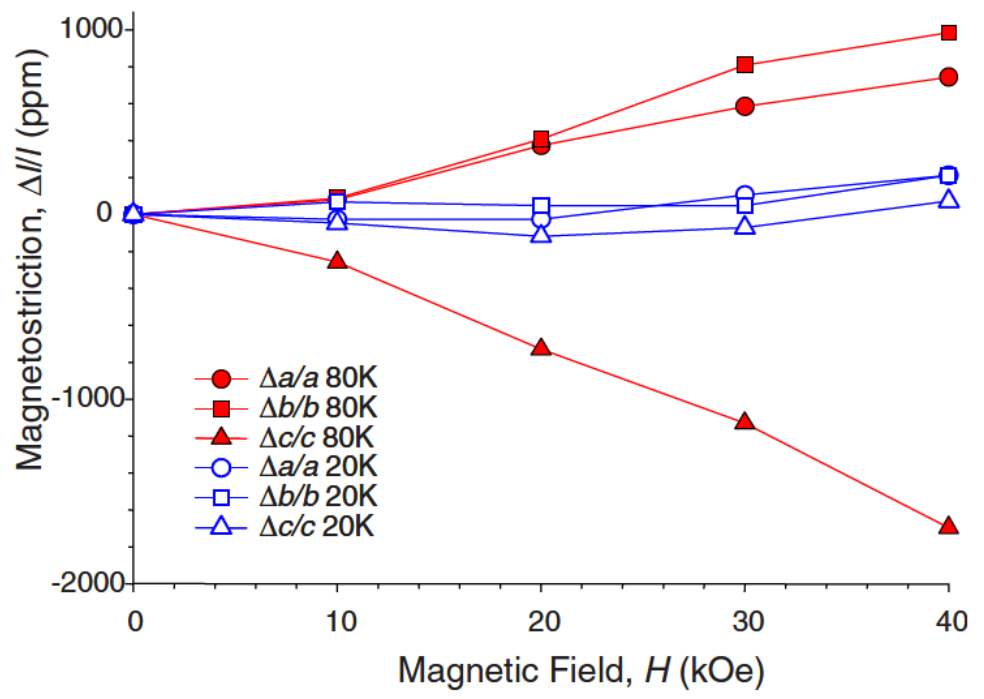

Figure 1.3: The magnetostrictive effect in GdNi shown in ppm strain. Magnetostriction is active only near the Curie temperature (69K) (taken from Ref. 17). 


\subsection{Magnetocaloric effect}

The magnetocaloric effect (MCE) is the core principle behind magnetic refrigeration and was first discovered in $1881 .{ }^{27}$ The MCE is observed as an adiabatic temperature change when a magnetic field is applied to a magnetic material. Kumar et al. 2008, studied the effect in a series of RNi compounds and found polycrystalline GdNi to have an isothermal magnetic entropy change $\left(-\Delta \mathrm{S}_{\mathrm{M}}{ }^{\max }\right)$ of $17 \mathrm{~J} \mathrm{~kg}^{-1} \mathrm{~K}^{-1}$, which is comparable to other compounds with similar Curie temperatures. ${ }^{19}$

The total entropy of a rare earth magnetic material is the sum of lattice $\left(\mathrm{S}_{\mathrm{L}}\right)$, electronic $\left(\mathrm{S}_{\mathrm{E}}\right)$, and magnetic entropies $\left(\mathrm{S}_{\mathrm{E}}\right)$. Magnetic entropy is decreased by applying a magnetic field due to magnetic ordering. The process is adiabatic thus electronic and lattice entropies are raised to keep total entropy constant. The increases in $\mathrm{S}_{\mathrm{L}}$ and $\mathrm{S}_{\mathrm{E}}$ are seen as an increase in temperature known as heating by adiabatic magnetization, and the process is reversible, thus cooling by demagnetization also occurs when the field is removed. The derivation of the adiabatic temperature change and isothermal magnetic entropy change from total entropy follows.

$$
d S(H, T)=d\left(S_{L}(H, T)+S_{E}(H, T)+S_{M}(H, T)\right)
$$

Expanding each of the terms gives:

$$
\begin{aligned}
d S(H, T)=\left(\frac{\partial S_{L}(H, T)}{\partial T}\right)_{H} d T+\left(\frac{\partial S_{L}(H, T)}{\partial H}\right)_{T} d H+\left(\frac{\partial S_{E}(H, T)}{\partial T}\right)_{H} d T \\
+\left(\frac{\partial S_{E}(H, T)}{\partial H}\right)_{T} d H+\left(\frac{\partial S_{M}(H, T)}{\partial T}\right)_{H} d T+\left(\frac{\partial S_{M}(H, T)}{\partial H}\right)_{T} d H
\end{aligned}
$$

From the second law of thermodynamics and the definition of heat capacity the differential entropy becomes: 


$$
d S(H, T)=\frac{C_{L}(T)}{T} d T+\frac{C_{E}(T)}{T} d T+\frac{C_{M}(T)}{T} d T+\left(\frac{\partial S_{M}(H, T)}{\partial H}\right)_{T} d H
$$

where $C_{L}, C_{E}, C_{M}$ are the components of the total heat capacity. Combining heat capacity terms to total heat capacity $\mathrm{C}_{\mathrm{T}}$ and applying adiabatic conditions, where entropy change is zero yields:

$$
d S(H, T)=0=\frac{C_{T}(T)}{T} d T+\left(\frac{\partial S_{M}(H, T)}{\partial H}\right)_{T} d H
$$

The Maxwell equation

$$
\left(\frac{\partial S}{\partial H}\right)_{T}=\left(\frac{\partial M}{\partial T}\right)_{H}
$$

can be applied to the last term, and solving for $d T$ and combining heat capacity terms to $\mathrm{C}_{\mathrm{T}}$, total heat capacity the equation becomes:

$$
d T(T, H)=-\frac{T}{C_{T}(T, H)}\left(\frac{\partial S_{M}(H, T)}{\partial H}\right)_{T} d H=-\frac{T}{C_{T}(T, H)}\left(\frac{\partial M(H, T)}{\partial T}\right)_{H} d H
$$

Assuming an adiabatic magnetization process from $\mathrm{H}_{\mathrm{i}}$ to $\mathrm{H}_{\mathrm{f}}$, the temperature change is described as:

$$
\Delta T_{a d}(T, \Delta H)=-\int_{H_{i}}^{H_{f}} \frac{T}{C_{T}(T, H)}\left(\frac{\partial M(H, T)}{\partial T}\right)_{H} d H
$$

and the magnetic entropy change can be written as:

$$
\Delta S_{M}(T, \Delta H)=\int_{0}^{T} \frac{\left(C(T, H)_{H_{f}}-C(T, H)_{H_{i}}\right)}{T} d T
$$

\subsection{Magnetoresistance}

Magnetoresistance (MR) is observed as the change in electrical resistivity when a magnetic field is applied to a material. The magnetic field can either be applied 
longitudinally or transversely to the electric field. Magnetoresistance is defined as the percentage change in resistivity from the value of the zero field resistivity, shown in equation 9.

$$
M R=\frac{\rho_{H}-\rho_{0}}{\rho_{0}}
$$

This designation of magnetoresistance assigns an increase of resistivity in field as a positive value. The phenomenon is rooted in the way electrons behave in a magnetic field. In zero field, electrons travel in linear paths. In a magnetic field, and electron feels a force as the cross product of its velocity and the magnetic field, defined as the Lorentz force, shown in equation 10.

$$
F=q[\vec{E}+(\vec{v} \times \vec{B})]
$$

Where $F$ is the force on the particle, $q$ is the charge and $v$ is the velocity of the particle, and $E$ and $B$ are the electric and magnetic fields. The result is moving conduction electrons in a magnetic field will travel in a helical path, thus a positive magnetoresistance is commonly seen. In ferromagnetic materials, the applied magnetic field can increase the order of magnetic moments within a material and so reduce the scattering from magnons, giving a negative magnetoresistance.

\subsection{Experimental Motivation}

The primary goal at the outset of this study was to develop processes to produce large pristine single crystals for characterization measurements. The rare earth and transition metal composition of GdNi makes it incompatible with many common methods of processing, and the consequences of these incompatibilities affect the accuracy of the

characterizations to be made. Gadolinium bonds very strongly with oxygen, thus when 
molten gadolinium comes in contact with common oxide crucibles, such as alumina, yttria, and other oxides, it has the ability to strip oxygen out of the crucible, and the crucible material can contaminate or fail to contain the melt. Nickel alloys readily with refractory metals, such as tungsten and tantalum, which are other common crucible materials. To process single crystal GdNi, non-traditional crucibles or crucible-free growth methods must be used. The characterization measurements desired include magnetic, transport, and thermal properties. The more these properties of GdNi are understood, the unique characteristics of $\mathrm{GdNi}$ and related compounds can be manipulated and applied. Beyond understanding physical properties and the mechanisms behind such properties, the advances made in processing will open paths to synthesize and research more complex RNi compounds. The RNi system has proven itself to be a system with unique crystallography and many of these compounds have virtually unknown properties. ${ }^{28-30}$ Figure 1.4 shows the progression of crystal structures from GdNi, the CrB type, to $\mathrm{YNi}$ and $\mathrm{DyNi}$, the FeB type, achieved through rare earth substitutions in the RNi system. 

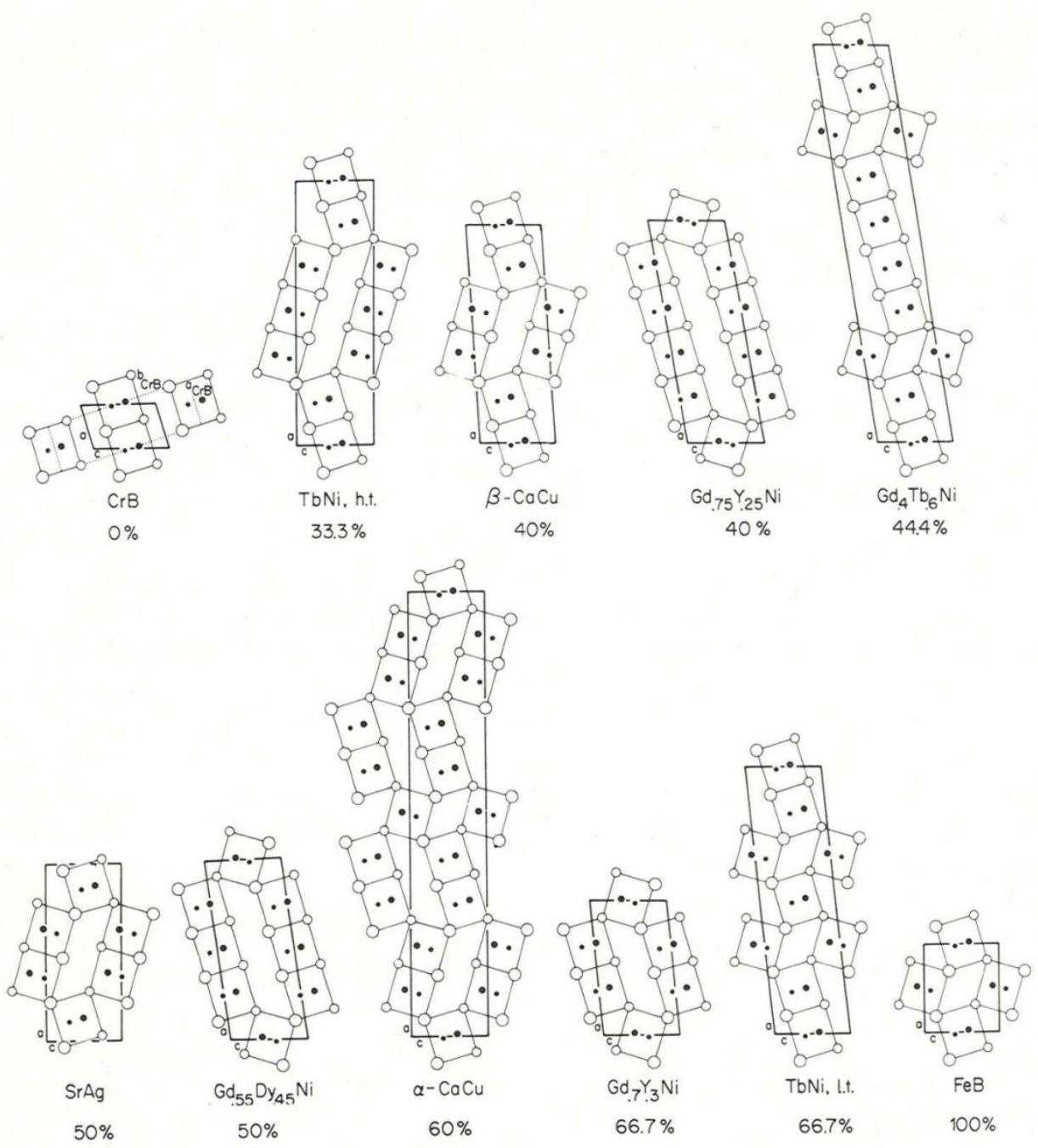

Figure 1.4: The progression of crystal structures of the RNi system (taken from Ref. 30) 


\section{Chapter 2 Experimental techniques}

\subsection{Materials sources and sample preparation}

The Gd used in characterization studies was prepared by the Ames Laboratory Materials Preparation Center. ${ }^{31}$ The Gd used was from one of two batches, Gd-007/Gd-008, and contained the following major impurities C, 589/314; N, 494/101; O, 1504/2486; and Al, 5/525 ppm atomic. H content was not tested but both batches of Gd are approximately 99.74/99.66 at.\% (99.98/99.96 wt.\%) pure considering all elements. For preliminary studies such as crucible compatibility testing, a commercially sourced Gd metal was occasionally used from Metall Rare Earth Limited; the purity was quoted 99.8 wt.\% Gd, with the main metallic impurities being the heavy rare earth metals, $\mathrm{Fe}, \mathrm{Ca}$, and $\mathrm{Si}$. Metall Rare Earth Limited Gd metal was not analyzed for C, N, O or H, all of which were expected to be significant impurities. The Ni was purchased from Miller and Company and was analyzed for $\mathrm{Co}, \mathrm{Fe}, \mathrm{C}, \mathrm{S}, \mathrm{Cu}, \mathrm{Sn}$, and $\mathrm{Pb}$ impurities and tested 99.99 wt.\% pure. In some experiments, $\mathrm{B}, \mathrm{La}$, and $\mathrm{Y}$ were used in the preparation of Ta crucibles. The boron used was purchased from Alfa Aesar with 99.5 wt.\% purity. The lanthanum was from Rhone-Poulenc Chimie and labeled 99.9 wt.\% pure. Yttrium used was from Stanford Materials Corporation and was approximately 98.9 wt.\% pure. Since these materials were primarily used in crucible preparation processes purity was not the utmost concern.

All metal alloys were prepared by arc melting constituent materials in a water-cooled copper hearth under an argon atmosphere, purified with a Zr getter button. Alloys were turned and re-melted several times for compositional homogeneity. Depending on the 
process to be used, alloys were either left in button form or cast into cylindrical or conical copper molds for use in crucibles.

Tantalum tubes, rods, and sheets found various uses in many processing experiments. The tantalum was sourced from Sincemat Co., Ltd. and was 99.95\% tantalum. Alumina and yttria crucibles from Coors Tek were used and were manufactured by the slip-cast method. Plasma-sprayed crucibles were prepared by the Ames Lab Materials Preparation Center and were sprayed with yttria stabilized zirconia (YSZ) and $\mathrm{Gd}_{2} \mathrm{O}_{3}$. The $\mathrm{YSZ}$ was sourced from Praxair Surface Technology, ZRO-178, containing 9.0\% $\mathrm{Y}_{2} \mathrm{O}_{3}$ and the remainder $\mathrm{ZrO}_{2}$. $\mathrm{Gd}_{2} \mathrm{O}_{3}$ was produced by Triebacher Industrie AG and labeled 99.9\% pure.

\subsection{Experimental apparatuses: Processing}

\subsubsection{Tri-arc crystal pulling method}

The tri-arc furnace used for crystal growths was manufactured by Materials Research Furnaces Inc. The furnace is an arc melting furnace with three tungsten electrodes to be placed equidistantly around the circumference of the button. The furnace has a watercooled copper hearth preventing any reaction with the alloy. The environment is highpurity Ar and is further purified with two $\mathrm{Zr}$ getter buttons. A drawing rod for crystal pulling is mounted in the center of the furnace and can draw at rates between 0.423 and $212 \mu \mathrm{m} / \mathrm{s}$ and can be rotated, controlled electronically. A viewing glass exists to monitor crystal growth and a CCD camera is used to enlarge the view of the button. 


\subsubsection{Bridgman method}

An Oxy-Gon Industries Bridgman furnace was used for Bridgman growths and has a temperature range up to $2100^{\circ} \mathrm{C}$. The furnace has a turbomolecular vacuum pump for high vacuum evacuation and can be backfilled up to 100psig with high-purity Ar passed through a getter furnace. A drawing system with a stepper motor is used to remove crucibles from the heat zone and can be withdrawn as slowly as $1 \mathrm{~mm} / \mathrm{hr}$. The crucibles are mounted within a Ta tube placed over a water cooled support rod to provide a temperature gradient for consistent and predictable solidification.

\subsection{Experimental apparatuses: Characterization}

\subsubsection{Scanning electron microscope}

A scanning electron microscope (SEM) was utilized to view crucible interactions and identify phases. The SEM is a JEOL brand JSM-5910LV equipped with secondary and backscatter electron detectors and energy dispersive x-ray spectroscopy (EDS). The backscatter detection mode was preferentially used for imaging because of its excellent phase contrast. The EDS detector was used to qualitatively identify phases seen.

\subsubsection{Laue backscatter diffraction}

A Laue backscatter diffraction system was used to identify the single or polycrystalline nature of crystal growths and to orient single crystals. The Laue system has a molybdenum x-ray source and a Photonic Science CCD to capture diffraction patterns digitally. The sample mount is fixed and the sample goniometer is adjusted manually. 


\subsubsection{Powder x-ray diffraction}

A PANalytical X'Pert Powder X-ray diffractometer was used to analyze phase purity. GdNi powder samples initially collected from the same region of crystal harvest and were prepared with a mortar and pestle and passed through a \#400 (38 $\mu \mathrm{m})$ mesh to ensure uniform particle size. The powders were spread on a zero background Si wafer and rotated during scans. The $\mathrm{X}$-ray source was copper $\mathrm{K} \alpha$ radiation and scans were performed at room temperature.

\subsubsection{Magnetic property measurement system}

Magnetization measurements were made in a superconducting quantum interference device (SQUID) magnetometer; Quantum Design, Inc. manufactured MPMS-XL. All magnetization measurements followed a zero field cooling (ZFC) procedure, starting well above the Curie temperature and cooling down to $2 \mathrm{~K}$ in zero-field to begin each measurement. Data reported along different crystallographic directions from a specific growth method were all collected from a single sample, remounted between measurements. The errors of the measurements taken are temperature, $0.5 \%$, magnetic field, 1Oe, and magnetic moment, $0.1 \%$.

\subsubsection{Physical property measurement system}

A physical property measurement system (PPMS) manufactured by Quantum Design, Inc. with $\mathrm{AC}$ transport attachment was utilized to measure temperature dependent resistivity in zero field and in an applied magnetic field to calculate magnetoresistance. Long parallelepiped samples were prepared and resistivity was measured via four-point measurement. Platinum leads $25 \mu \mathrm{m}$ in diameter were attached to the sample with silver 
epoxy and samples adhered to measurement pucks with GE varnish. Resistivity $(\rho)$ is defined as:

$$
\rho=\frac{A}{l} R
$$

where $A$ is the cross-sectional area, $l$ is the separation between voltage measurement leads and $R$ is measured resistance. Ohm's law defines resistance $(R)$ as:

$$
R=\frac{V}{I}
$$

The applied magnetic field, which has an error of 10e; and the voltage measurement and applied current of $25 \mu \mathrm{A}$, used to calculate resistivity, both have an error of $0.1 \%$. The largest error in the measurement comes from the uncertainty of the lead separation and cross-sectional area accounting for about a $15 \%$ error, depending on sample dimensions.

\subsubsection{Heat capacity measurements}

Heat capacity was measured with a custom built automated heat pulse calorimeter. ${ }^{32}$ Samples mounted are parallel polished and mounted with silver paste to aid thermal conductivity between the sample stage and the sample. The errors of the heat capacity measurements are less than $0.5 \%$. The calorimeter is mounted within a superconducting magnet capable of producing fields up to 100kOe. Heat capacity, field and temperature are measured to calculate the magnetocaloric effect. 


\section{Chapter 3 Single crystal growth attempts by tri-arc crystal pulling method}

The tri-arc crystal pulling method proved to be a challenging method of crystal growth for $\mathrm{GdNi}$. The relatively low melting temperature of $\mathrm{GdNi}$ at $1280^{\circ} \mathrm{C}$ required a low power setting on the power supply and compromised the stability of the arcs. Larger mass buttons, $45 \mathrm{~g}$, up from $20 \mathrm{~g}$, were used in an attempt to require more power and increase the run stability. A larger Ta pullrod was also used to pull crystals to allow more heat flow out of the button, in an effort to further increase the operating power and thus the arc stability. The lower temperature metal also has less intense and lower energy blackbody radiation. The radiation from the button indicates the temperature and the liquid or solid state of the button. The challenge of the lower melting temperature is that the arcs are very bright and wash out the radiation from the button making the state of the button difficult to observe.

Three GdNi samples were drawn in the tri-arc using the crystal pulling method as can be seen in Figure 3.1. The pullrod was not rotated and drawn at $4.23 \mu \mathrm{m}$ per second. Unfortunately the samples drawn were all polycrystalline. One of the growths resulted with grains close to $1 \times 1 \times 1 \mathrm{~mm}^{3}$ but the crystals too small to orient and cut for magnetization measurements. The challenging run parameters made a good growth difficult because the diameter of the ingot was not easy to control. Ideally, when a growth begins, the diameter is reduced by controlling the temperature and pull rate, to isolate a single grain, and then the growth parameters are altered to increase the diameter to grow 
a large single crystal. Considering the processing challenges, the tri-arc crystal pulling method was not pursued further in favor of more promising crystal growth methods.
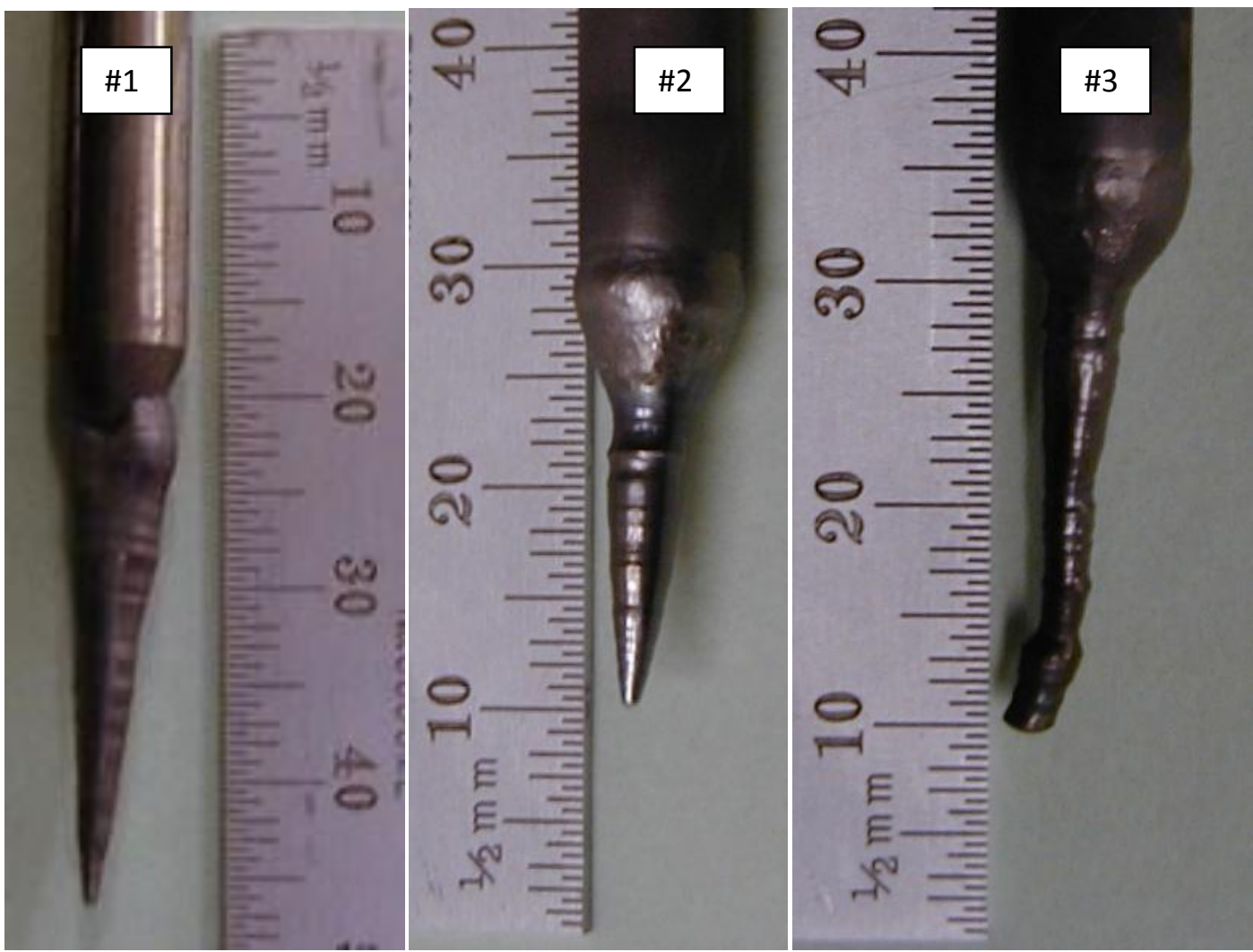

Figure 3.1: Three GdNi samples drawn in a tri-arc in the crystal pulling method. The diameters of the growths were difficult to control, thus the samples generally taper down to a point. All samples are polycrystalline, but \#3 had the largest grains of approximately $1 \times 1 \times 1 \mathrm{~mm}^{3}$, though still not large enough for characterization studies. 


\section{Chapter 4 Precipitation growth of GdNi}

Crystal growth of GdNi was attempted through self-flux or a precipitation growth. GdNi is a congruent melting compound, see Figure 4.1 for the phase diagram. ${ }^{33}$ It was discovered that cooling through the liquidus of Gd-rich off-stoichiometric Gd-Ni compounds, small GdNi crystals precipitate out of solution. $\mathrm{Gd}_{3} \mathrm{Ni}_{2}$ is a stable line compound and this was the nominal composition used for the precipitation of GdNi. $\mathrm{Gd}_{3} \mathrm{Ni}_{2}$ has a lower melting point than $\mathrm{GdNi}$ and lower nickel content. These factors allow pure tantalum tubes to be used as crucibles for precipitation growths of GdNi. To precipitate $\mathrm{GdNi}, \mathrm{Gd}_{3} \mathrm{Ni}_{2}$ was loaded into Ta crucibles with a decanting lid. The entire crucibles were then sealed in quartz tubes under high purity argon gas. The crucibles in quartz tubes were then heated in box furnaces to $1100^{\circ} \mathrm{C}$ to ensure a homogeneous melt before cooling. Cooling rates of $1-3 \mathrm{C} / \mathrm{hr}$ were found to produce plate-like crystals. At $730^{\circ} \mathrm{C}$ the crucibles are turned upside-down and centrifuged to separate the flux from the GdNi crystals. The result is small growth colonies approximately $0.7 \times 0.7 \times 5 \mathrm{~mm}^{3}$. The crystals grow long in the $a$ direction and have a rhombus shaped cross-section. Cooling through the temperature range at $1 \mathrm{C} / \mathrm{hr}$ takes over 15 days. Slower rates of cooling could not be achieved due to furnace power instability over several week time scales. 


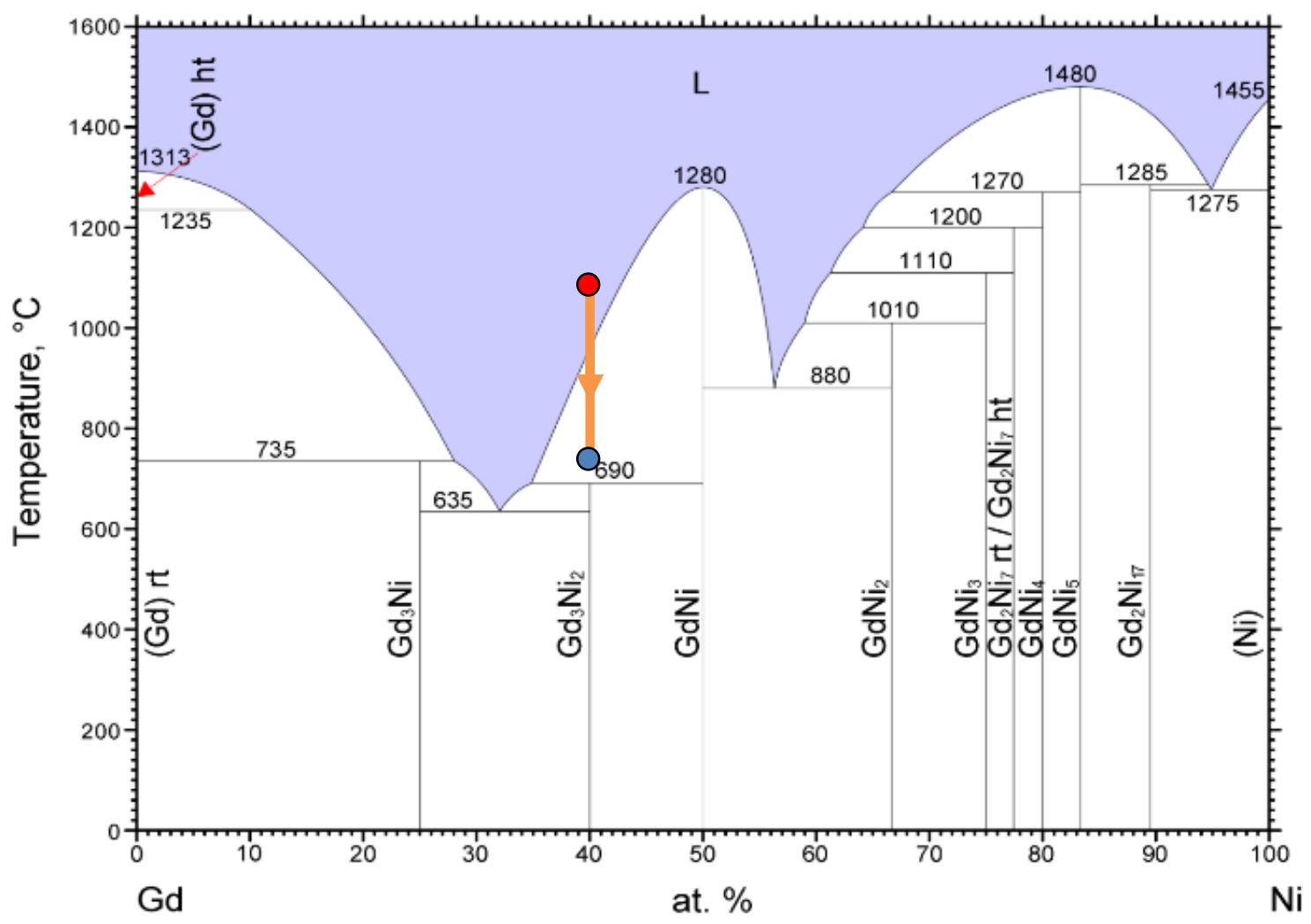

Figure 4.1: The Gd-Ni phase diagram (taken from Ref. 33). Indicated on the diagram is the self-flux crystal growth experiment. The $\mathrm{Gd}_{3} \mathrm{Ni}_{2}$ starts at $1100^{\circ} \mathrm{C}$ (red dot) and cools slowly to $730^{\circ} \mathrm{C}$ (blue dot), where the remaining liquid is poured away from the GdNi crystals.

Flux grown GdNi plates grow in the $\left(\begin{array}{lll}0 & 2 & 1\end{array}\right)$ family of crystallographic planes identified by Laue backscatter x-ray diffraction. In the GdNi system there are $78.3^{\circ}$ and $101.7^{\circ}$

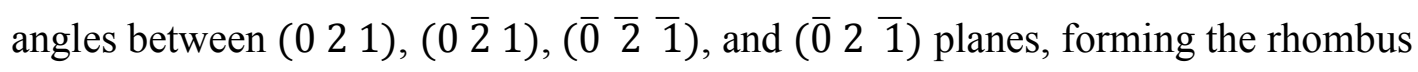
cross-section. GdNi crystals were prepared for magnetization measurements in a SQUID magnetometer. The anisotropic shape of the crystals only allowed the $a$-axis direction to be measured but an impurity phase, $\mathrm{Gd}_{3} \mathrm{Ni}$, was also present. An additional magnetic transition was seen in the magnetization vs. temperature $(\mathrm{M}(\mathrm{T}))$ measurement in Figure 4.2. The remaining flux is a eutectic mixture of $\mathrm{Gd}_{3} \mathrm{Ni}$ and $\mathrm{Gd}_{3} \mathrm{Ni}_{2} \cdot \mathrm{Gd}_{3} \mathrm{Ni}$ has a known magnetic transition at $\sim 100 \mathrm{~K}$ and is seen in the magnetic data. ${ }^{34}$ Figure 4.2 has an arrow indicating the $\mathrm{Gd}_{3} \mathrm{Ni}$ magnetic transition. SEM imaging of the flux grown crystal shows 
flux trapped in the GdNi crystal in Figure 4.3, explaining why the magnetic transition is seen near 100K. The long and delicate processing, unavoidable trapped impurities, and limited size and shape of crystals proved flux growth to not be a preferable single crystal processing method for producing GdNi crystals for property measurements.

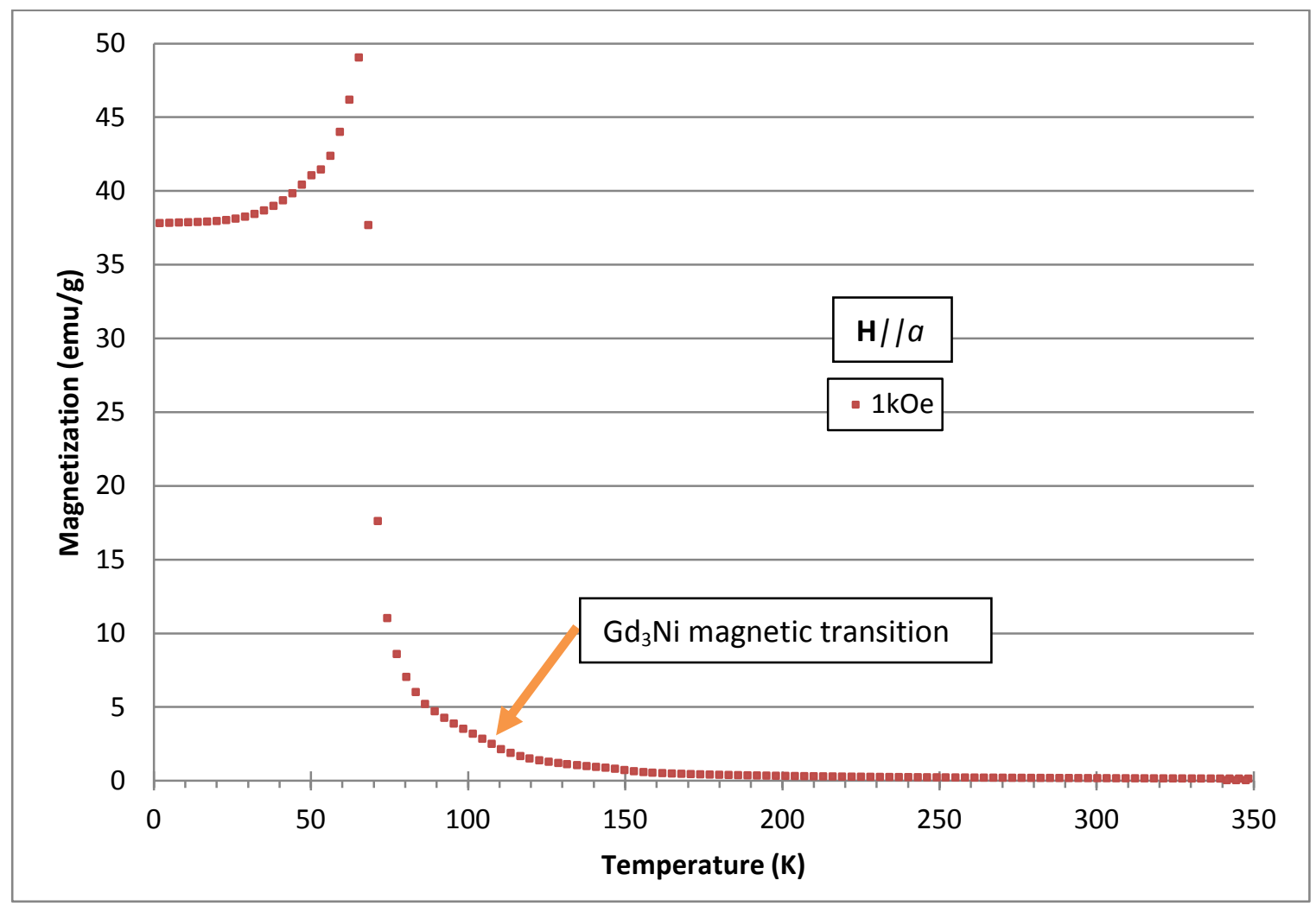

Figure 4.2: The M(T) curve of a flux-grown GdNi single crystal along the a direction in a $1 \mathrm{kOe}$ field. An additional transition is seen near $100 \mathrm{~K}$ attributed to $\mathrm{Gd}_{3} \mathrm{Ni}$, found in the flux used to grow crystals (see Figure 4.3). 


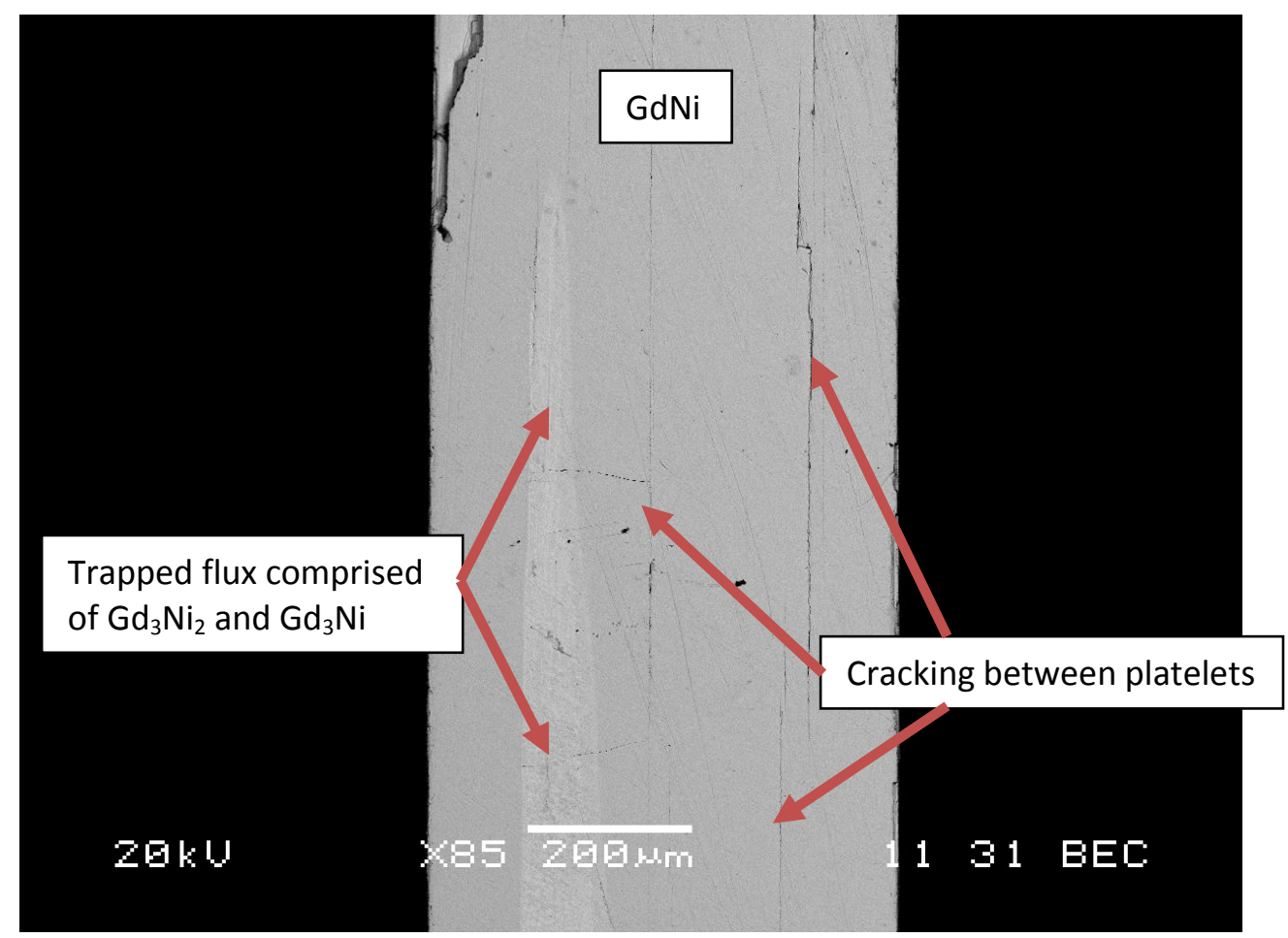

Figure 4.3: An SEM image viewing a flux grown GdNi single crystal. Trapped flux can be seen within the crystal. The planar nature of the plate-like crystals is illustrated by the vertical cracking in the crystal, separating individual platelets that joined to form a larger crystal. 


\section{Chapter 5 GdNi Processing via commercially available crucibles}

\subsection{Yttria crucible crystal growth results}

A Bridgman crystal growth was attempted in yttria, heating to $1425^{\circ} \mathrm{C}$ under $40 \mathrm{psi}$ gettered Ar and drawing at $10 \mathrm{~mm} / \mathrm{hr}$. The growth was found to be contaminated with $\mathrm{Y}$. A qualitative EDS analysis and SEM imaging indicated roughly a 5\% content of $\mathrm{Y}$ in the melt and $\mathrm{GdNi}_{2}$ impurity phase was also present. SEM imaging also showed the crucible reaction. Figures 5.1 and 5.2 show the interaction between GdNi and the $\mathrm{Y}_{2} \mathrm{O}_{3}$ crucible. The reaction zone was quite extensive and had a thickness of roughly $1 \mathrm{~mm}$, half the original crucible wall dimension. The reaction zone contains many Gd-Y-Ni-O compounds and the $\mathrm{Y}_{2} \mathrm{O}_{3}$ can be seen dissolving into the melt. Some of the phases identified were Gd substituting into $\mathrm{Y}_{2} \mathrm{O}_{3}$, specifically $\left(\mathrm{Gd}_{1-x} \mathrm{Y}_{x}\right)_{2} \mathrm{O}_{3}$ where $x \approx 0.5-0.9$, and Y substituting into GdNi and $\mathrm{GdNi}_{2}$ specifically $\mathrm{Gd}_{1-x} \mathrm{Y}_{x} \mathrm{Ni}$ and $\mathrm{Gd}_{1-x} \mathrm{Y}_{x} \mathrm{Ni}_{2}$ where $x \approx 0.1$. Y contaminating the melt can alter the crystal structure and change the Curie temperature. YNi has a FeB (Pnma) crystal structure and Parthe et al. found varying crystal structures when substituting Y for Gd in the GdNi system ${ }^{28-30}$ and Mallik et al. studied many properties of $\mathrm{Gd}_{1-x} \mathrm{Y}_{x} \mathrm{Ni}$ alloys, reporting lower $\mathrm{T}_{\mathrm{C}}$ with $\mathrm{Y}$ substitution. ${ }^{35}$ 


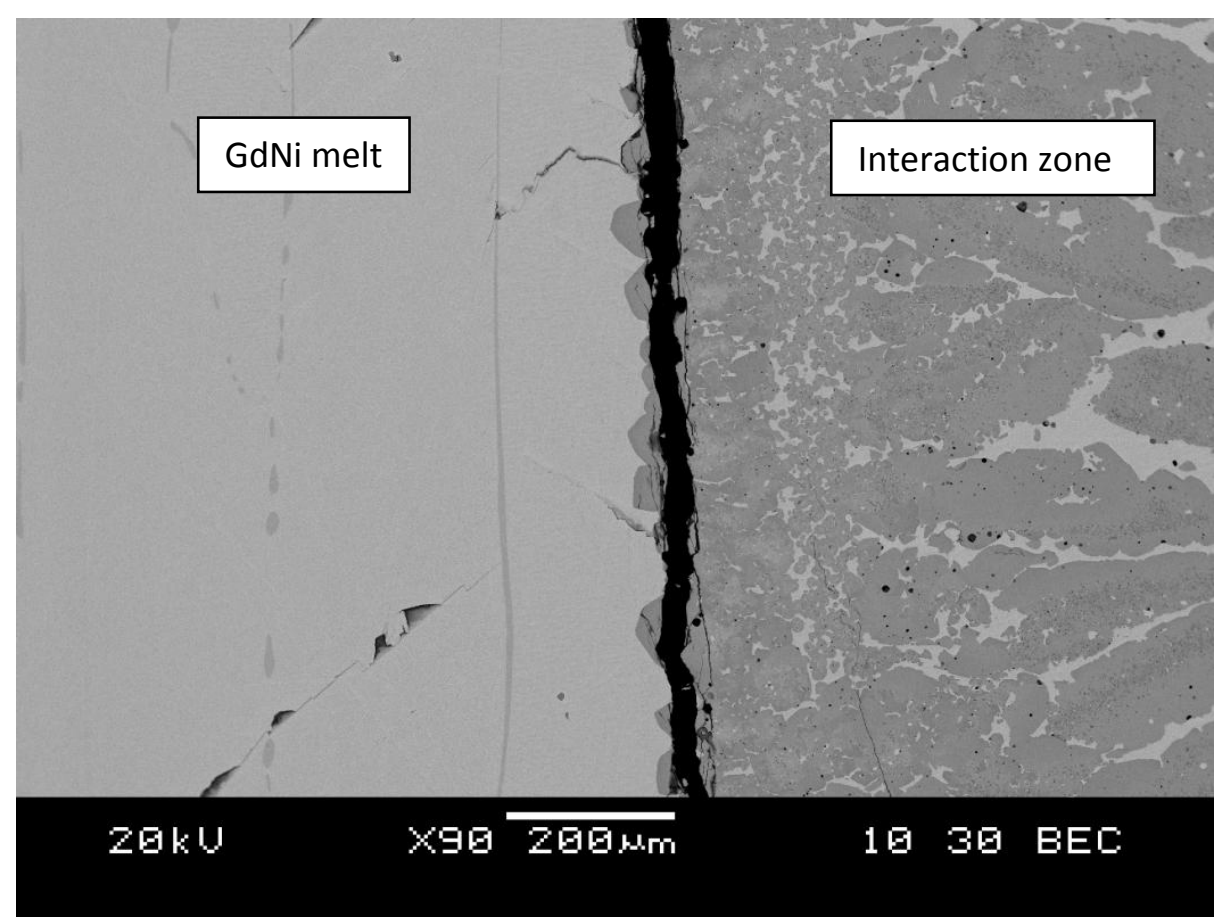

Figure 5.1: The GdNi melt and interaction zone interface. $\mathrm{GdNi}_{2}$ impurity is seen in the melt. The interaction zone contains Gd and $Y$ oxides and many Gd-Y-Ni compounds. The melt was solidified from bottom to top.

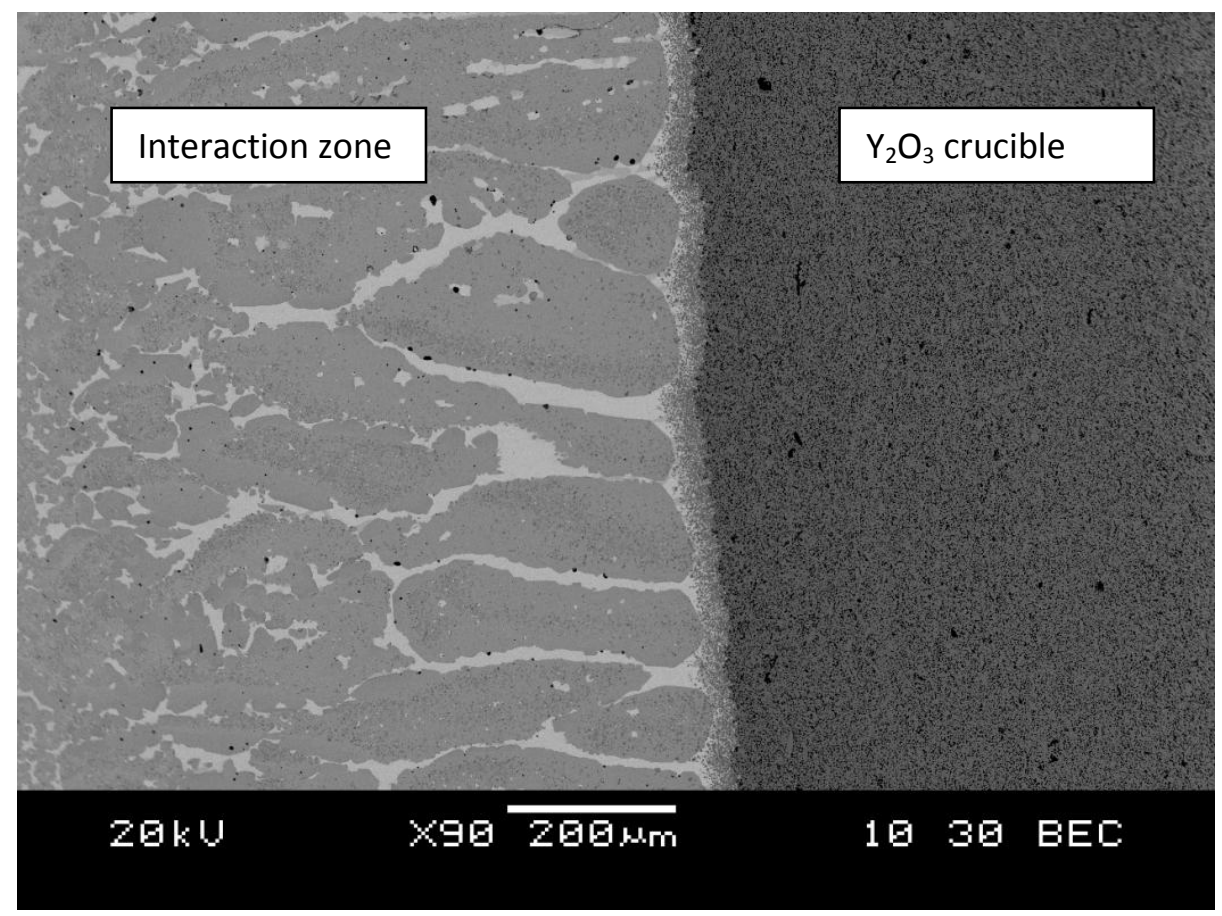

Figure 5.2: The interaction zone and $\mathrm{Y}_{2} \mathrm{O}_{3}$ interface. The image area is translated slightly vertically and to the right of the area seen in Figure 3.1. The interaction zone contains many impurity phases. 


\subsection{Alumina crucible crystal growth results}

A Bridgman growth was attempted in an alumina crucible because several publications cite using alumina crucibles successfully. ${ }^{6,7,8,36}$ Due to an acute Gd shortage, Y was substituted and the experiment ran with YNi in an alumina crucible. The furnace was heated to $1425^{\circ} \mathrm{C}$, the running temperature of GdNi growths, and withdrawn at $7 \mathrm{~mm} / \mathrm{hr}$. The reaction was strong enough to determine alumina crucibles could not be used to achieve the goal of this study. An SEM image of the growth can be seen in Figure 5.3. The phases seen are qualitatively identified with EDS. Based on the intense reaction of yttrium with alumina, alumina was determined to not be a proper crucible for the preparation of GdNi.

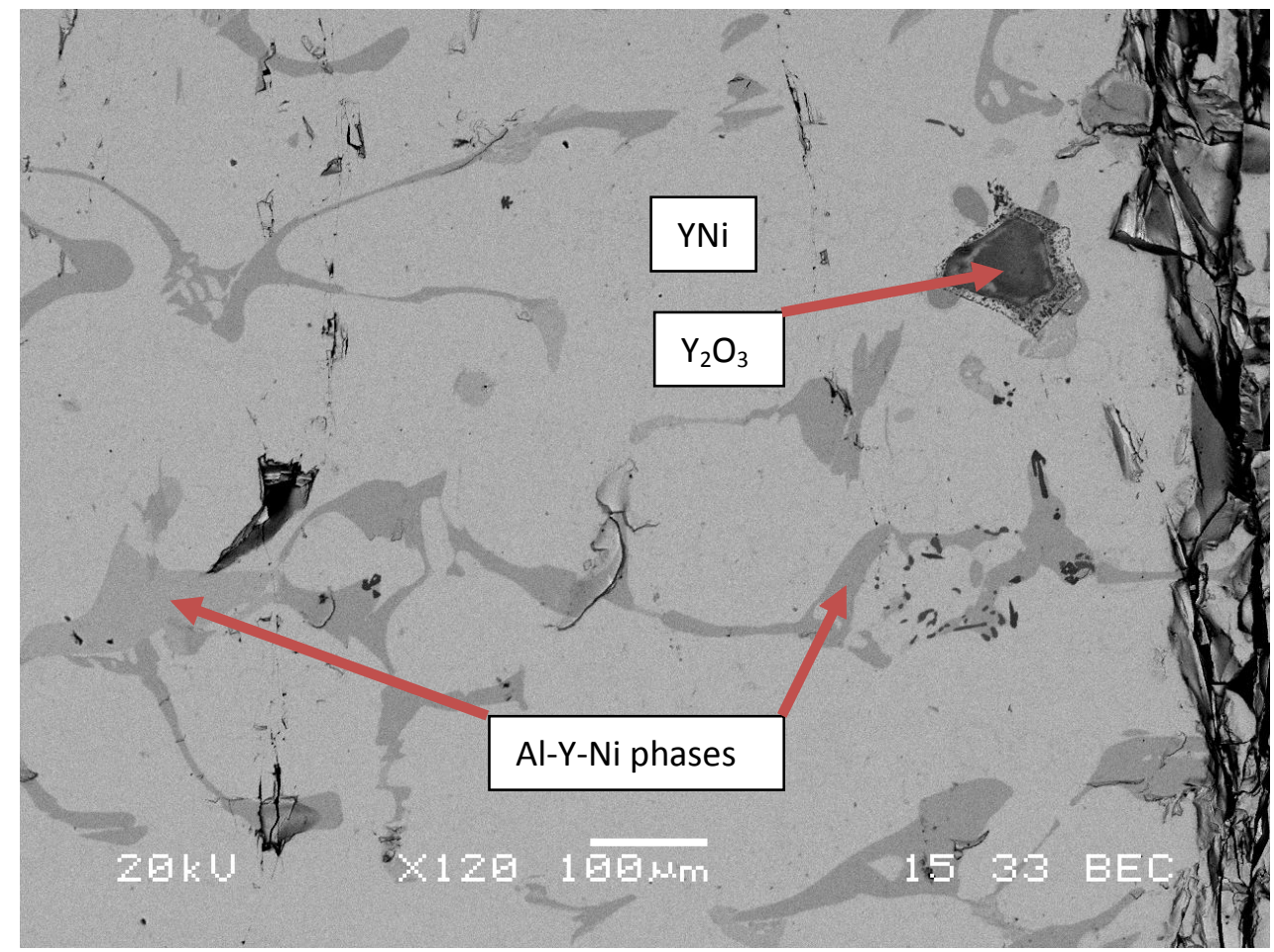

Figure 5.3: An image of YNi grown in an alumina crucible. The melt was solidified from right to left. Oxides and off-stoichiometry phases are present. The medium grey phases are Al-Y-Ni impurity phases. 


\section{Chapter 6 GdNi Processing via coated Ta crucibles results}

\subsection{Coated Ta crucible development}

Tantalum carbide and tantalum boride coatings applied to tantalum were tested and utilized as a robust and inert crucible for GdNi processing. The procedure for manufacturing coated tantalum crucibles was adapted from Gschneidner and Schmidt. ${ }^{37}$ The procedure used to coat a tantalum crucible is as follows:

- Etch tantalum crucible in 2 parts $12 \mathrm{M}$ hydrofluoric acid, 2 parts $12 \mathrm{M}$ hydrochloric acid, 1 part $12 \mathrm{M}$ nitric acid to prep surface for coating.

- Load and seal crucible with lanthanum plus $2.0 \mathrm{wt} . \%$ boron or $2.5 \mathrm{wt} . \%$ carbon.

- Heat in vacuum furnace to $1400-1900^{\circ} \mathrm{C}$ for 24 hours depending on coating thickness desired. For $\mathrm{GdNi}$, coatings were formed at $1600^{\circ} \mathrm{C}$ for 24 hours.

- Etch the lanthanum mixture away with $3 \mathrm{M}$ hydrochloric acid.

- Fire crucible at $1600^{\circ} \mathrm{C}$ for 1 hour under vacuum to remove hydrogen impurity from acid etching.

- Cut off uncoated section of crucible to leave a fully coated crucible interior. For GdNi it was determined a coated cap was not necessary and an uncoated tantalum cap has no effect on the success of the run. Tantalum caps can also be coated during the coating forming process by including a cap within the crucible and the coating has no effect on welding to seal a crucible. The coatings are known to form in two layers. The inner layer, in contact with $\mathrm{Ta}$, is $\mathrm{Ta}_{2} \mathrm{C}$ and the outer layer, in contact with the crucible contents, is TaC. The boride coating forms in the same manner but the actual phases have not been identified. 
Using this process, carbide and boride coated test crucibles were made. The crucibles were loaded with $\mathrm{GdNi}$ and heated to $1400^{\circ} \mathrm{C}$ for 12 hours to mimic the running conditions of a Bridgman crystal growth. From this experiment it was observed through viewing in the SEM the carbide crucible would not be suitable for GdNi for the coating had partially failed and both tantalum and carbon had contaminated the melt. The boride coated crucible remained intact and showed promise as a crucible for the Bridgman method. Figures 6.1 and 6.2 show the carbide and boride coating interactions with GdNi from the test crucibles.

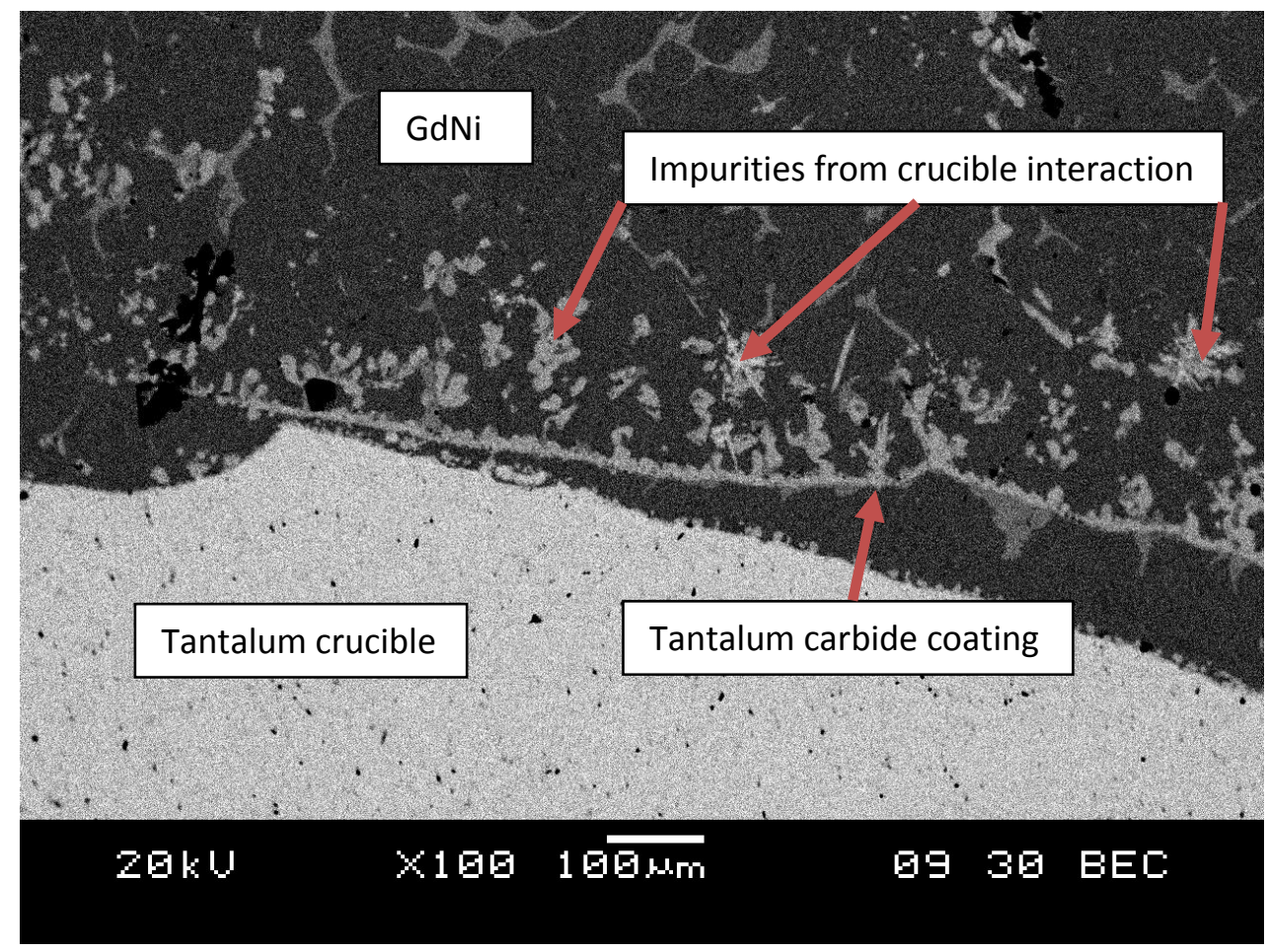

Figure 6.1: The bottom of a tantalum carbide coated tantalum crucible with GdNi melt. The coating can be seen separated from the crucible and the tantalum beneath eroded. Many carbon and tantalum based impurity phases are also seen in the melt. 


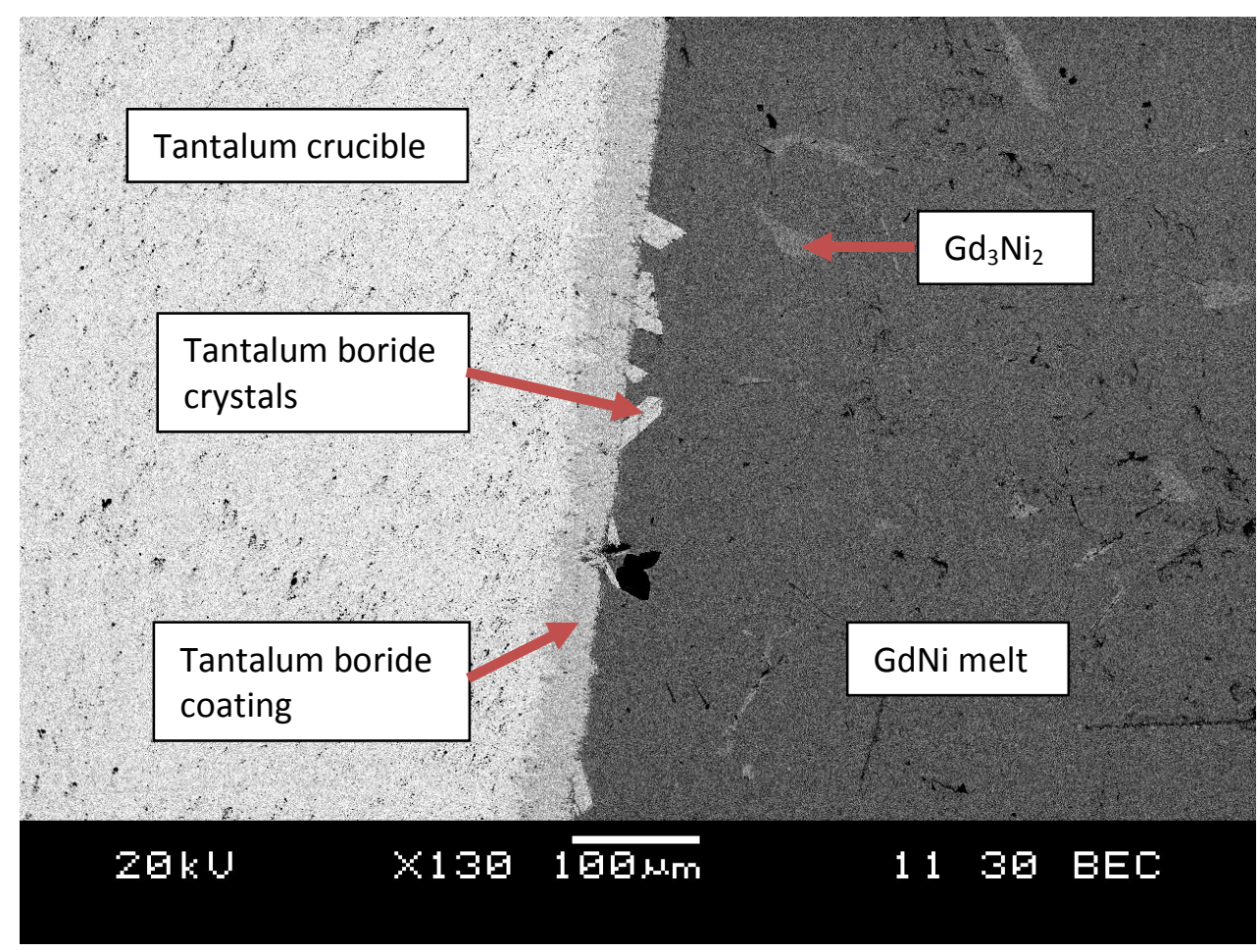

Figure 6.2: The wall of a tantalum boride coated tantalum crucible with GdNi. The coating remained intact and no major impurities are seen in the melt. Some tantalum boride crystals are also seen growing off the coating but were not seen elsewhere within the melt. The melt impurity seen is the $\mathbf{G d}_{3} \mathbf{N i}_{2}$ phase from slightly $\mathbf{G d}$-rich starting materials.

A Bridgman growth in a pointed-bottom boride-coated tantalum crucible, made from a $12.7 \mathrm{~mm}$ diameter tantalum tube, was executed in an attempt to grow single crystal GdNi. The purpose of the pointed bottom is to solidify a very small volume of material first, thus minimizing the points of nucleation to ideally nucleate one crystal that will grow throughout the crucible. The experiment was run at $1425^{\circ} \mathrm{C}$, under 40 psi argon, drawing at $10 \mathrm{~mm} /$ hour. The result was not a successful crystal growth. The growth resulted in a polycrystalline sample which was heavily fractured likely due to coefficient of thermal expansion mismatch between the GdNi and the crucible. Using optical microscopy, it was easy to see an impurity phase had formed (Figure 6.3) that was not detected by SEM backscatter imaging. The impurity was determined to be a gadolinium boride compound 
formed from excess boron as the boron-rich layer of the coating converts to the lowerboron-content layer in the crucible coating.

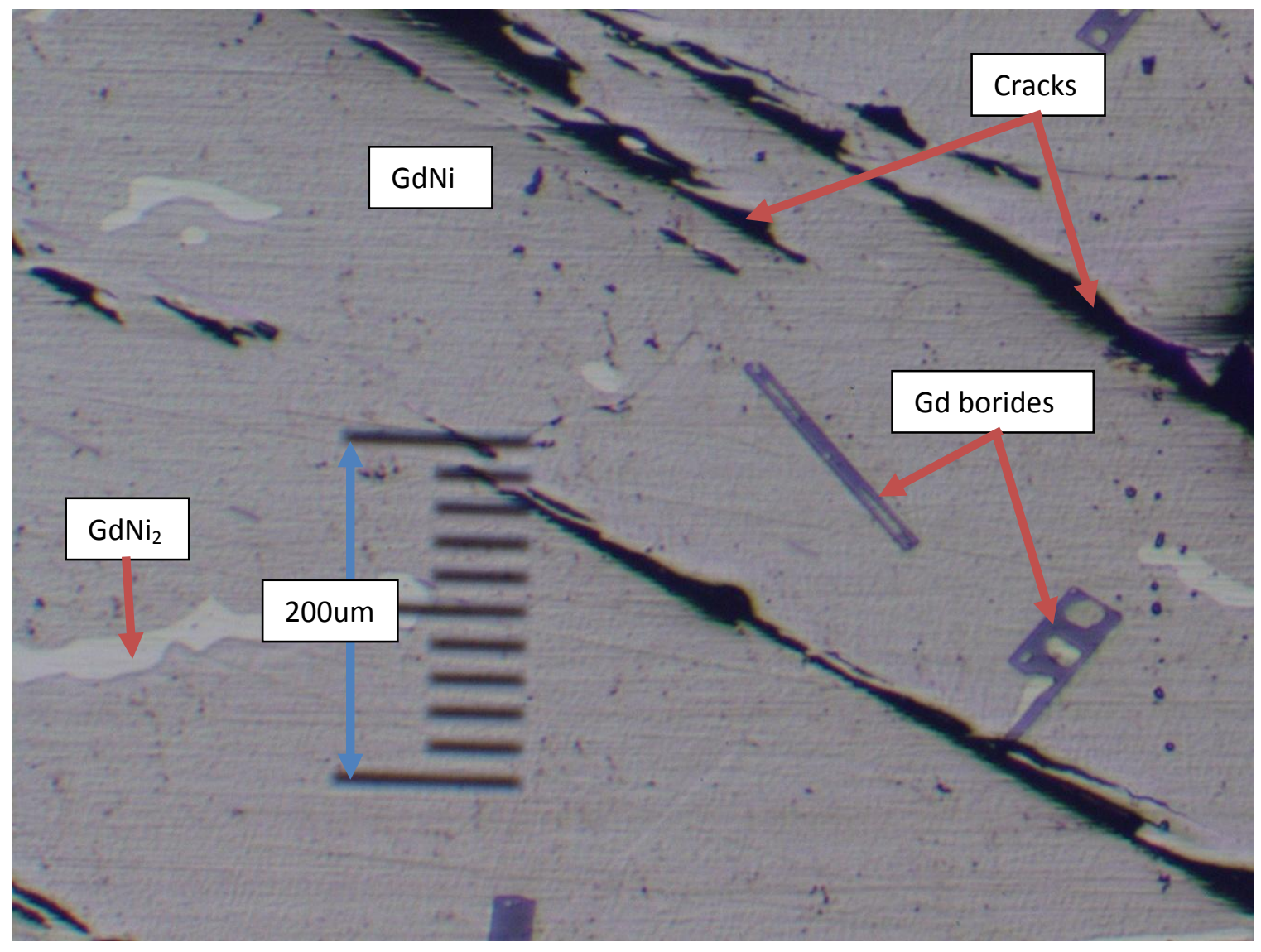

Figure 6.3: GdNi single crystal growth in tantalum boride coated tantalum, scale bar - 200um. The dark rectangular shapes are gadolinium borides. The light phase is $\mathrm{GdNi}_{2}$. The sample also shows cracks from stress related to thermal expansion.

Since the boride coating was found release boron into $\mathrm{GdNi}$, an additional processing step was devised to remove the additional boron to create an inert crucible for GdNi. After etching the lanthanum boron mixture out of the tantalum crucible, the following steps are added:

- Load newly coated crucible with yttrium, seal and heat in vacuum furnace at $1600^{\circ} \mathrm{C}$ for 1 hour.

- $\quad$ Etch out $\mathrm{Y}$ with $3 \mathrm{M} \mathrm{HCl}$. 
The process material selected was Y because it is a relatively inexpensive rare earth metal to pull the excess boron out of the tantalum boride coating, like what Gd had been observed to do. What is assumed to be happening during this additional processing is the outer tantalum boride layer, containing a higher atomic percentage of $\mathrm{B}$, is converted to the inner tantalum boride layer. The atomic ratios of the tantalum boride layers have not been identified but Gschneidner and Schmidt reported a similar conversion to occur in

the $\mathrm{TaC} / \mathrm{Ta}_{2} \mathrm{C}$ coatings when processed with $\mathrm{Gd} .{ }^{37}$ The result of the additional processing with $\mathrm{Y}$ is a crucible suitable for GdNi single crystal growth.

\subsection{Coated Ta crucible crystal growth results}

GdNi single crystals were successfully grown in tantalum boride coated tantalum. The crucible was welded closed under high purity argon at a pressure level such that the operating pressure would be approximately 40 psi at the growth temperature. The Bridgman run started at $1425^{\circ} \mathrm{C}$ and the crucible was drawn at $7 \mathrm{~mm} / \mathrm{hr}$. Figures $6.6-6.8$ show the interfaces and melt in detail. The additional processing was found to slightly weaken the coating. Most of the coating remained intact and no gadolinium borides were detected but near the meniscus the coating did fail and tantalum contaminated the melt. The crucible did not completely fail, and the pure Ta beneath the tantalum boride coating contained the melt while only eroding slightly. The Ta was found to alloy with $\mathrm{Ni}$ forming Ni-Ta compounds with Gd also present but some mostly pure Ta particles were also found. The Ta compounds being denser than the GdNi melt largely sank to the bottom of the crucible and disrupted the crystal growth, leading to additional crystal nucleation. Roughly the top half of the growth had very few Ta impurities and only 2-3 
major crystal orientations. Tantalum and Ni-Ta alloys are both paramagnetic, and in the small quantity present do not significantly affect the magnetization of GdNi.

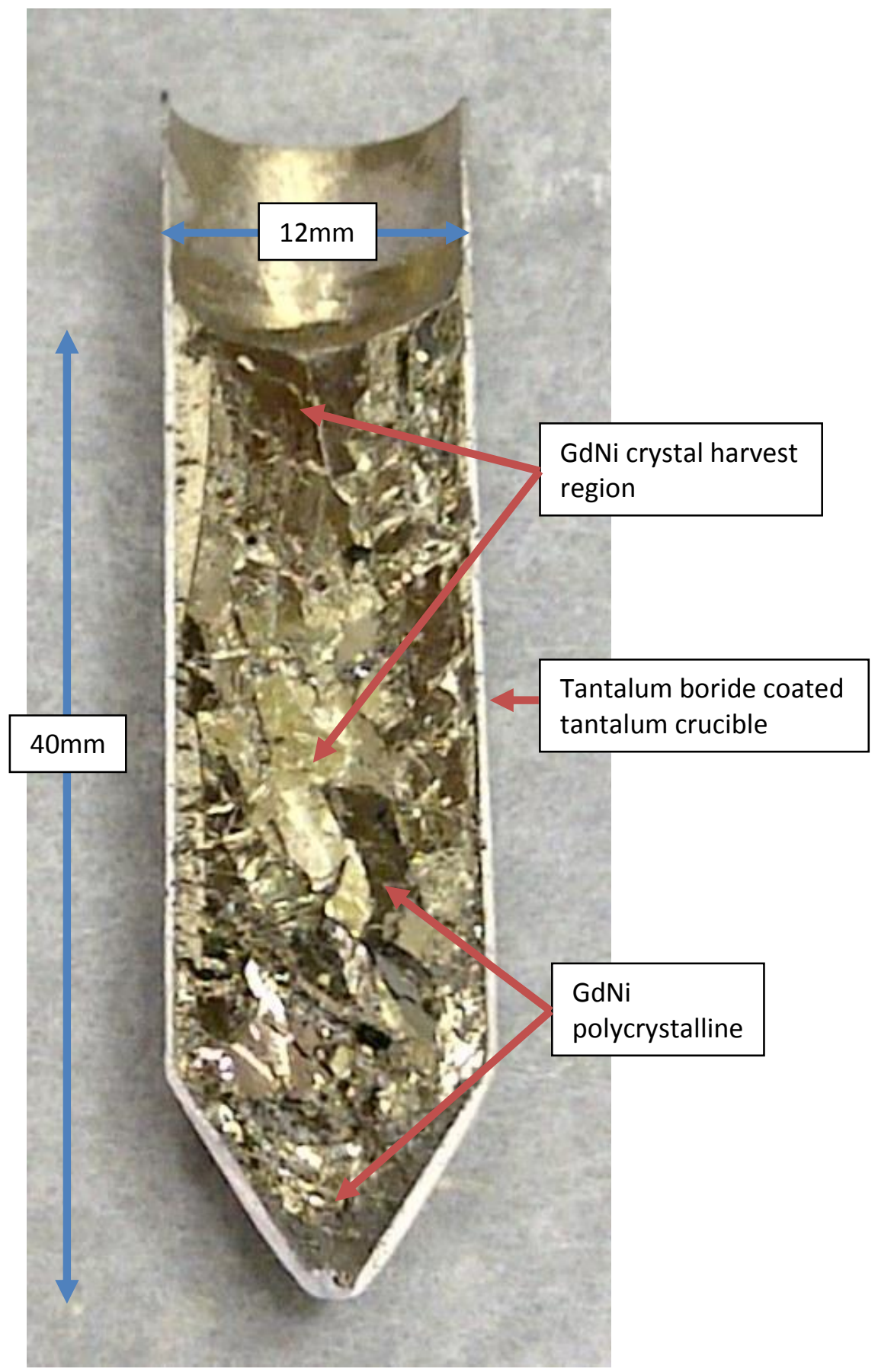

Figure 6.4: A cross-section of a GdNi crystal growth in a tantalum boride coated tantalum crucible. The crucible was cut along a natural fracture in the growth and the $b$ plane is primarily seen. The bottom half of the growth has numerous crystal orientations; the upper half of the crucible has only a couple orientations. 


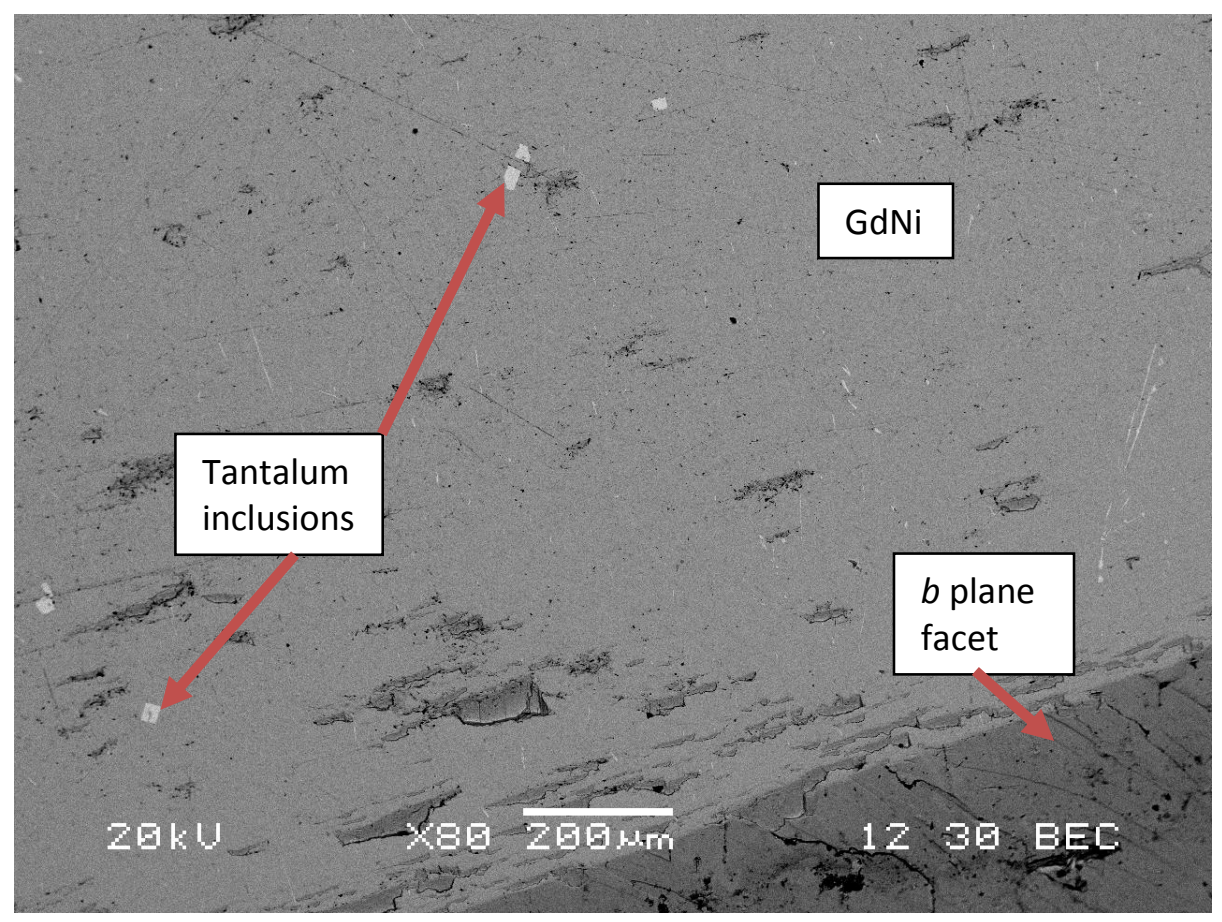

Figure 6.5: A polished single crystal of GdNi grown in tantalum boride coated tantalum in SEM. In the bottom right a $b$ facet is seen. Tantalum impurities are seen in the melt as the light phase.

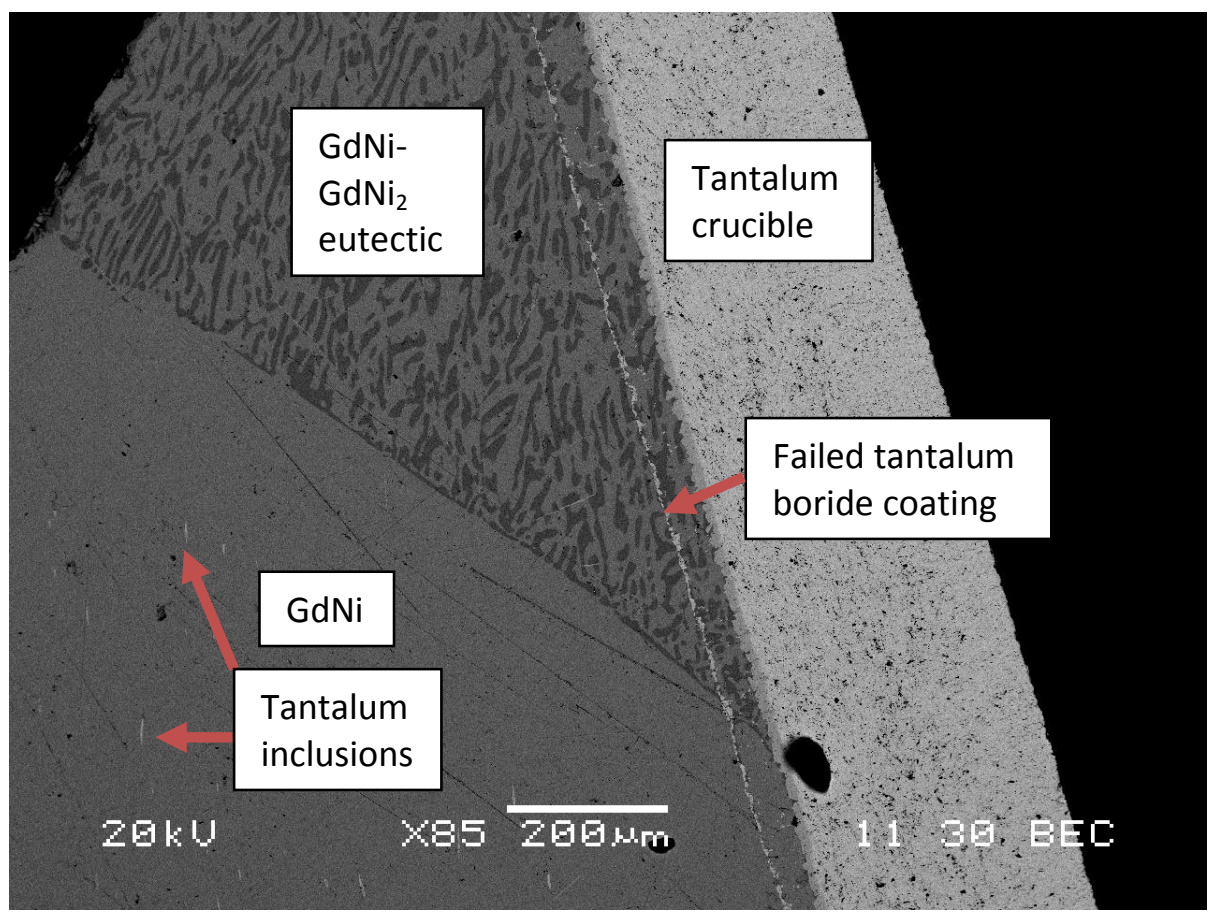

Figure 6.6: A cross-section SEM image of a tantalum boride coated tantalum crucible and GdNi crystal growth near the meniscus. The coating is separated from the tantalum crucible. Near the meniscus also remains the small volume of off-stoichiometry $\mathbf{G d N i}_{2}$ that solidified last. Small tantalum impurities in the melt can also be seen. 
From the upper half of the crucible, single crystals were harvested. One crystal specimen, roughly $1 \mathrm{x} 1 \mathrm{x} 1 \mathrm{~mm}^{3}$, was oriented and cut for magnetization measurements. Another crystal was found to have grown along the $a$ direction and was cut to approximately $14 \times 1 \times 1 \mathrm{~mm}^{3}$. This crystal was further cut down to two $7 \mathrm{x} 1 \mathrm{x} 1 \mathrm{~mm}^{3}$ samples for resistivity measurements. The crystals were found to be limited in size due to cleavage along the $b$ direction, the dimension to about $2.3 \mathrm{~mm}$. In the $c$ direction, dimensions up to $5 \mathrm{~mm}$ had been measured, and in the $a$ direction, a $14 \mathrm{~mm}$ crystal was harvested, but dimensions of 6-8mm were much more common. A single crystal was also powdered and XRD was used to analyze phase purity. The XRD data and fit can be seen in Figure 6.7. There are no impurity phases seen, and the lattice parameters were measured to be $a=3.7659 \pm 0.0004 \AA, b=10.3099 \pm 0.0012 \AA$, and $c=4.2417 \pm 0.0006 \AA$ and the Rietveld refinement fit parameters were $R_{p}=5.44 \%, R_{w p}=6.09 \%, R_{\text {exp }}=4.99 \%$, and $\chi^{2}=1.49$. The lattice parameters show some deviation from previously published values, but as seen in Table 6.1, there is some variation within the GdNi unit cell. Historically, the unit cell dimensions of GdNi have varied by as much as $0.9 \%$. The $R_{p}, R_{w p}, R_{\text {exp }}$, and $\chi^{2}$ values of the Rietveld refinement, indicating the deviation between the experimental and the theoretically calculated diffraction patterns, are tolerable. Ideally the fit parameters should be minimized to a value close to 1 ; the $\mathrm{R}$ values are maybe a little large but for general lattice parameter measurements and phase purity analysis, the fit is acceptable. 


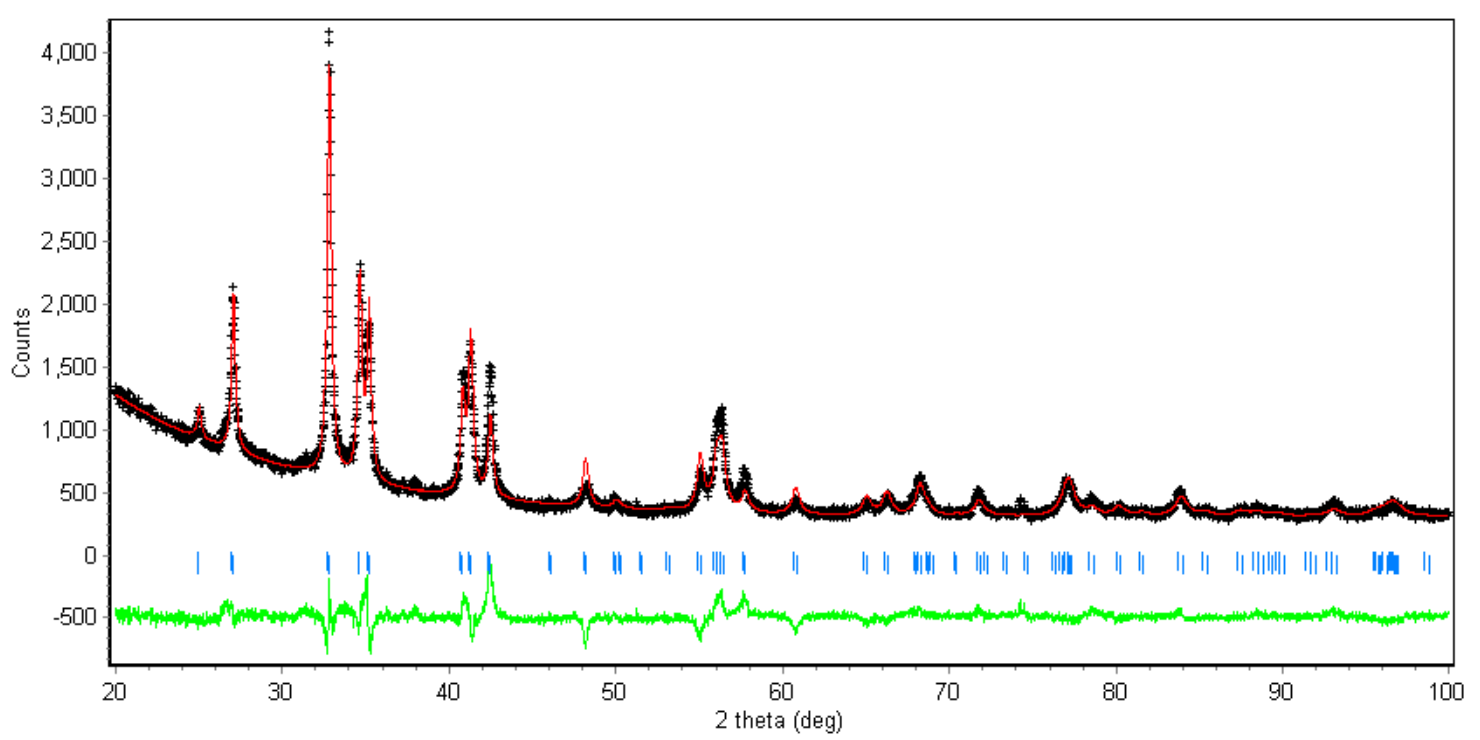

Figure 6.7: The Rietveld refinement and fit of GdNi powder from a single crystal grown in a tantalum boride coated tantalum crucible. No impurity phases are seen. A calculated XRD pattern (red) is fitted to the data (black). The blue bars indicate where GdNi peaks are calculated to exist. The green line indicates the difference between the calculated fit and the data.

Table 6.1: A historical representation of the lattice parameters of GdNi at room temperature. The measured lattice parameters have a variation up to $0.9 \%$.

\begin{tabular}{|c|c|c|c|c|c|}
\hline$a(\AA)$ & $b(\AA)$ & $c(\AA)$ & $\begin{array}{c}\text { Author } \\
(\text { et al. })\end{array}$ & Year & Ref. \\
\hline 3.771 & 10.327 & 4.248 & Paudyal & 2008 & 17 \\
\hline 3.767 & 10.306 & 4.239 & Baranov & 2008 & 18 \\
\hline 3.774 & 10.327 & 4.238 & Uhlirova & 2006 & 16 \\
\hline 3.751 & 10.275 & 4.232 & Paccard & 2005 & 38 \\
\hline 3.778 & 10.365 & 4.221 & Blanco & 1992 & 10 \\
\hline 3.752 & 10.310 & 4.252 & Ursu & 1972 & 5 \\
\hline 3.766 & 10.316 & 4.244 & Dwight & 1965 & 39 \\
\hline
\end{tabular}




\section{Chapter 7 GdNi Processing via plasma-sprayed powder crucibles}

\subsection{Plasma spray process crucible preparation}

The Ames Laboratory Materials Preparation Center has developed a specific plasma spray facility for the production of special ceramic crucibles, coatings, and spherical particles. ${ }^{31}$ Utilizing the Ames Laboratory Plasma Spray Facility, two crucibles, an yttria stabilized zirconia (YSZ) test crucible and a $\mathrm{Gd}_{2} \mathrm{O}_{3}$ lined, W-backed Bridgman crucible, were fabricated to test compatibility with GdNi and to grow GdNi single crystals. Plasma spraying is a semi-automated process in which a fugitive graphite mandrel is used as a crucible form. Graphite rods are typically used to shape the inner wall of the crucible and can be machined to specification. The mandrels are spun with an electric motor at a constant angular velocity. Shields are used to control the spray distribution and spraying is done by hand. Each pass adds a consistent thickness of material and thus coatings can be sprayed with a reasonable degree of thickness accuracy. After spraying, to remove the graphite mandrel, the crucibles are baked out in a box furnace a $900^{\circ} \mathrm{C}$ to slowly combust the graphite mandrel, leaving only the crucible behind. The $\mathrm{W}$ backed crucibles must spray $\mathrm{W}$ after burning out the graphite otherwise the $\mathrm{W}$ would oxidize. The crucible liner is simply slipped over another mandrel to complete the plasma spraying process.

The YSZ crucible was sprayed onto a straight $9.5 \mathrm{~mm}$ diameter graphite rod forming the inner diameter of the crucible. The wall thickness was sprayed to approximately $5 \mathrm{~mm}$ and the crucible had a depth of $18 \mathrm{~mm}$. The $\mathrm{Gd}_{2} \mathrm{O}_{3}-\mathrm{W}$ crucible was sprayed onto a tapered, pointed-bottom mandrel matching the measurements of other common Bridgman crucibles. The crucible is $100 \mathrm{~mm}$ long with a diameter of $22 \mathrm{~mm}$ at the top with a gradual 
taper to a $19 \mathrm{~mm}$ diameter near the bottom. The crucible was tapered sharply to a point approximately $20 \mathrm{~mm}$ from the bottom of the crucible.

\subsection{YSZ crucible processing results}

The YSZ test crucible had a strong reaction with GdNi and reacted in a unique way which can be seen in Figure 7.1. The crucible reacted with the Gd in the melt and the Gd pulled oxygen out of the crucible. The result is a heavily Gd deficient melt fully surrounded by a primarily gadolinium oxide shell. A nontrivial amount of Y and Zr also was found to have contaminated melt from substitution with Gd. The formation of the oxide layer, found to have completely surrounded the melt suggests the oxide layer was liquid at temperature $\left(1425^{\circ} \mathrm{C}\right)$ and solidified first, followed by the GdNi melt, Thus we see the melt pulled away from the oxide shell. The exact phase composition of the shell remains unknown. The oxides of Gd, Y, and $\mathrm{Zr}$ are all high melting, well over $2000^{\circ} \mathrm{C}$, so it is suspected that the materials present must have formed a eutectic with the low melting oxide and the melt. 


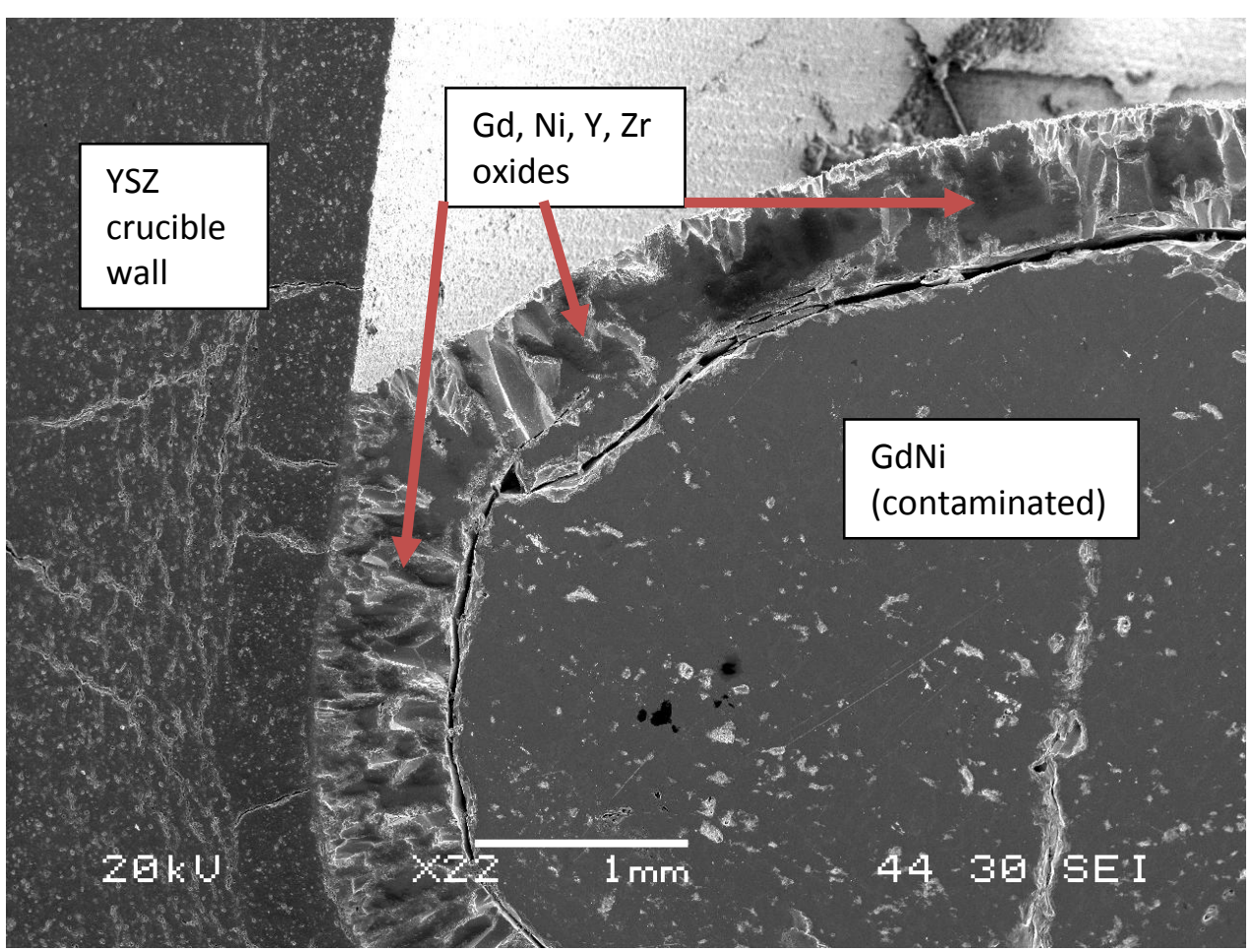

Figure 7.1: A cross-section SEM image of GdNi in a YSZ test crucible. A coating of oxides has formed around the melt and the melt is heavily contaminated.

\section{3 $\mathrm{Gd}_{2} \mathrm{O}_{3}$ crucible crystal growth results}

The $\mathrm{Gd}_{2} \mathrm{O}_{3}$ crucible was first baked out at $1600^{\circ} \mathrm{C}$ under vacuum to ensure the crucible is clean and also attempt to convert the $\mathrm{Gd}_{2} \mathrm{O}_{3}$ wall to a sub-oxide. The purpose of the suboxide is to prevent GdNi from pulling oxygen out of the crucible. Because of the layered construction, even at temperatures up to $1850^{\circ} \mathrm{C}$ the full conversion to sub-oxide could not be achieved. A casting of GdNi was inserted into the cleaned crucible and loaded into the Bridgman furnace. The furnace was pressurized to 40psi with gettered Ar gas, heated to $1425^{\circ} \mathrm{C}$, and the crucible was withdrawn at $7 \mathrm{~mm} / \mathrm{hr}$. The $\mathrm{Gd}_{2} \mathrm{O}_{3}$ crucible yielded a remarkably clean crystal growth with a few crystal orientations. A crystal growth can be seen in Figure 7.2. 


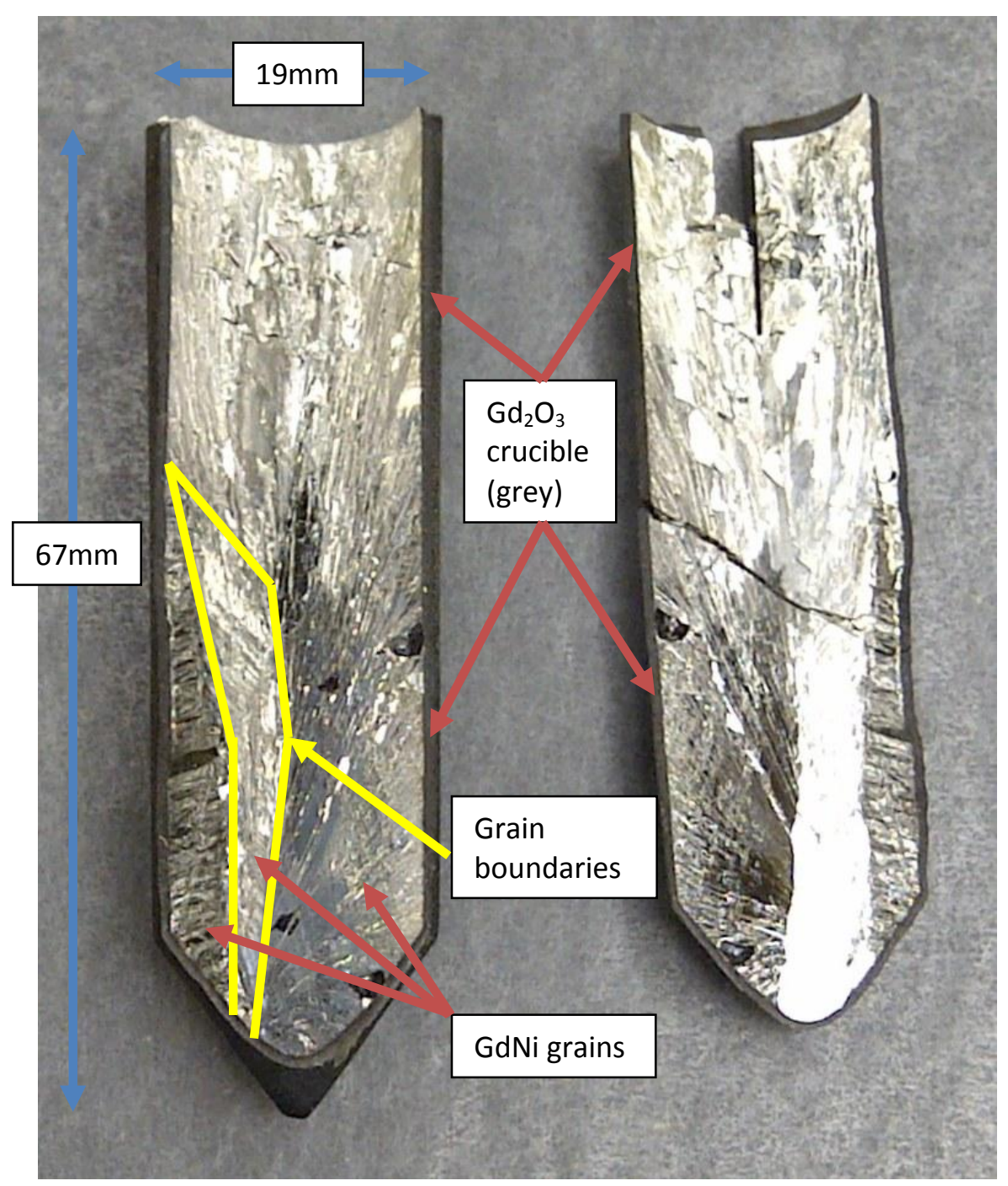

Figure 7.2: A cross-section image of a GdNi crystal growth in $\mathrm{Gd}_{2} \mathrm{O}_{3}$. The section was formed by natural cleavage along the $b$ plane. The crystal growth is clean and three orientations can be seen in the lower half of the crucible. The grain boundaries are highlighted in yellow. The $\mathrm{Gd}_{2} \mathrm{O}_{3}$ crucible bonded to the melt and turned to a sub-oxide grey color.

The crystals grown in $\mathrm{Gd}_{2} \mathrm{O}_{3}$ were limited in size by $b$ plane cleavage, and $b$-axis dimensions were measured up to $4 \mathrm{~mm}$. The $a$-axis dimensions were measured up to $20 \mathrm{~mm}$ and $c$-axis dimensions up to $10 \mathrm{~mm}$. An approximately $1 \times 1 \times 1 \mathrm{~mm}^{3}$ single crystal was cut and oriented along the major crystallographic directions for magnetization measurements. A $2.4 \mathrm{~mm}$ thick sample along the $b$-axis direction was prepared for heat capacity measurements. The other dimensions were approximately $6 \times 10 \mathrm{~mm}^{2}$. SEM 
imaging was used view potential single crystals for harvest and crucible wall interactions in Figures 7.3 and 7.4. The single crystal region shows some very sparse $\mathrm{Gd}_{2} \mathrm{O}_{3}$ impurities less than $5 \mu \mathrm{m}$ in size. On the crucible wall, some $\mathrm{Gd}_{2} \mathrm{O}_{3}$ dendrites are seen because of the crucible wall converting to a sub-oxide from contact with the Gd in the melt. Unlike other crucibles, there is no reaction zone and there are no additional contaminants from the crucible. Near the meniscus there was a small but noticeable amount of crucible erosion seen in Figure 7.5 picturing a separated section of the crucible wall.

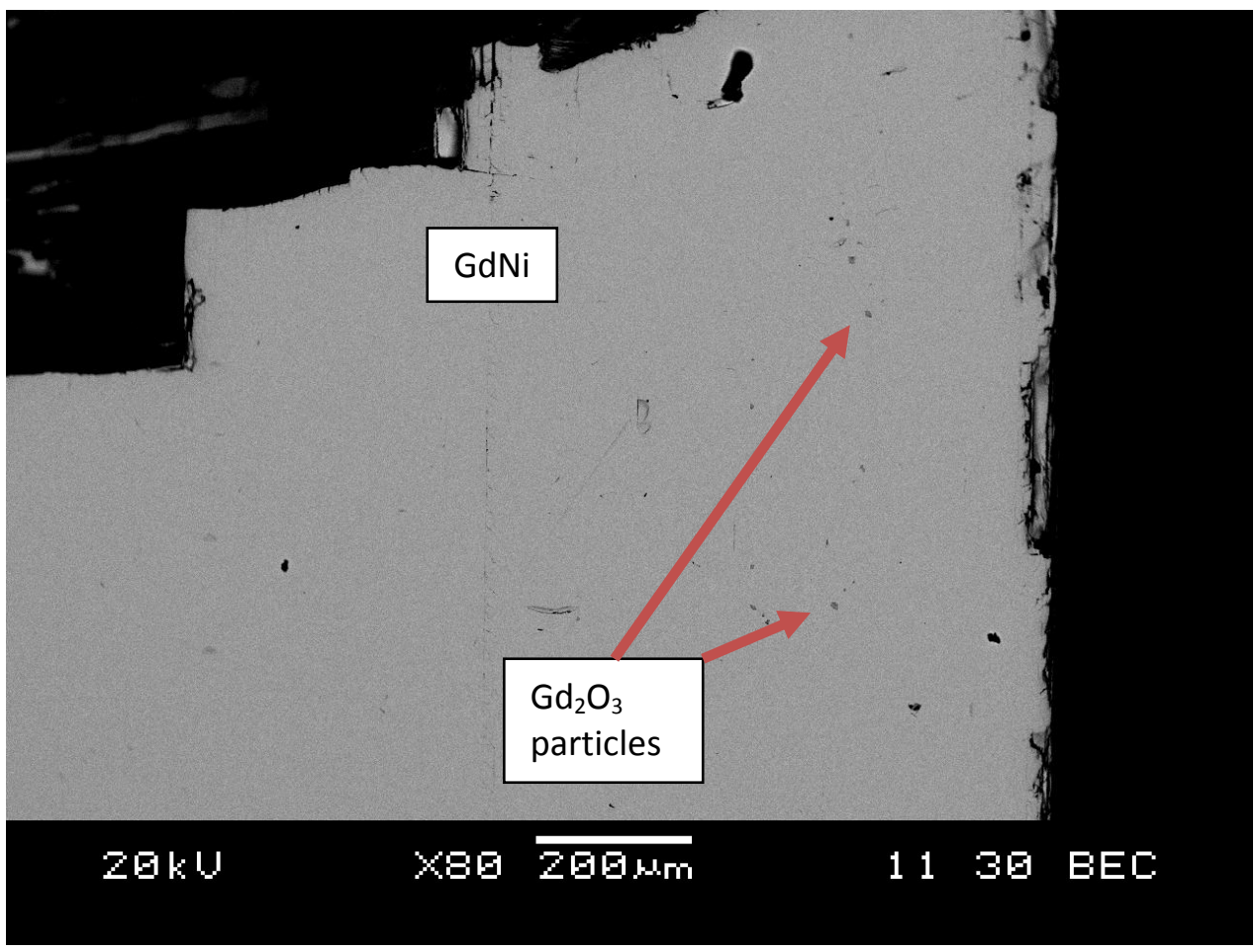

Figure 7.3: SEM image of a polished single crystal of GdNi grown in $\mathrm{Gd}_{2} \mathrm{O}_{3}$. On the right side of the crystal a few small $\mathrm{Gd}_{2} \mathrm{O}_{3}$ particles can be seen. 


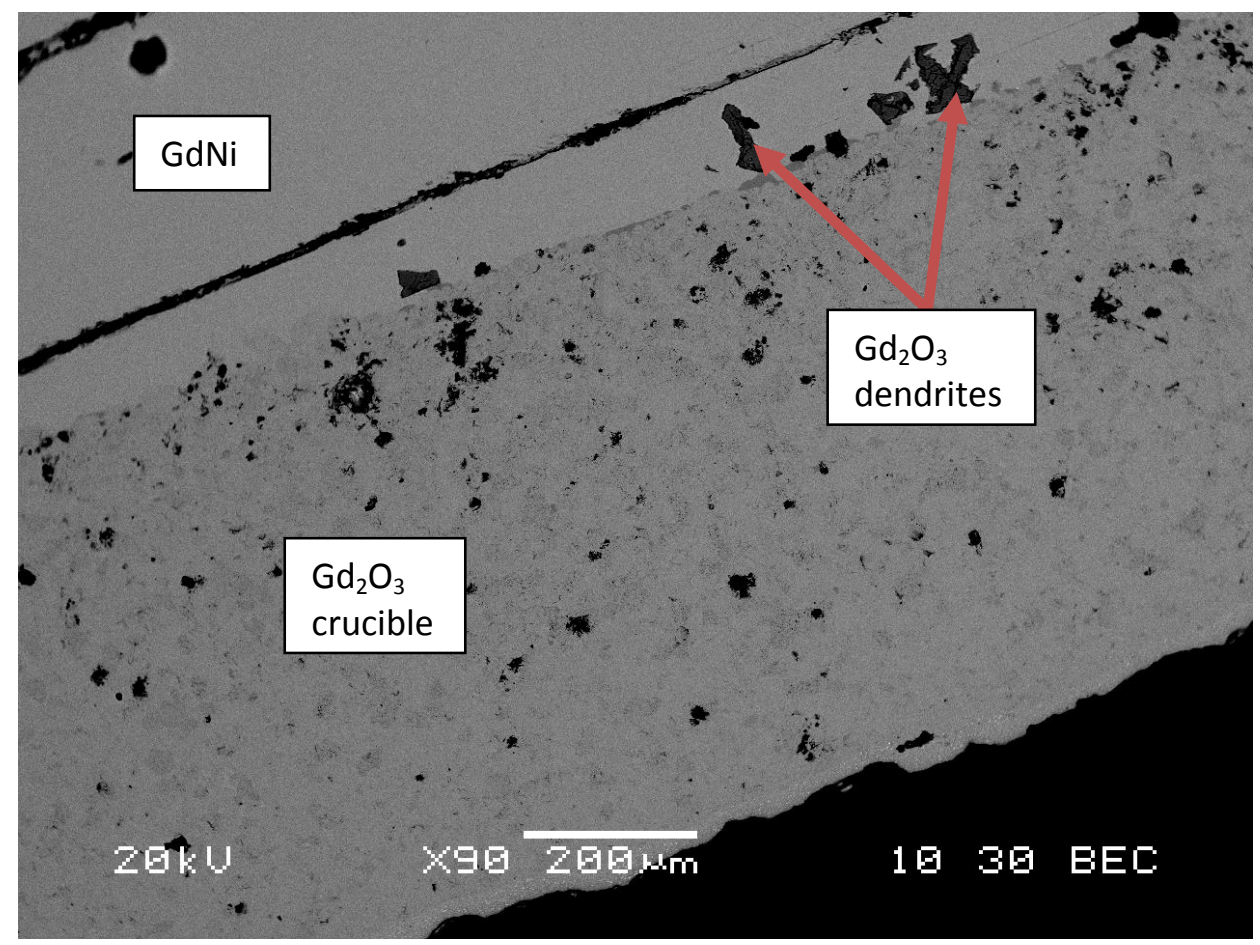

Figure 7.4: $\mathrm{The} \mathrm{Gd}_{2} \mathrm{O}_{3}$ crucible wall. The interaction with the melt is very minimal, only a few $\mathrm{Gd}_{2} \mathrm{O}_{3}$ dendrites are present. The $\mathrm{W}$ backing has been separated from the $\mathrm{Gd}_{2} \mathrm{O}_{3}$, which otherwise would be seen in the black region in the lower right corner.

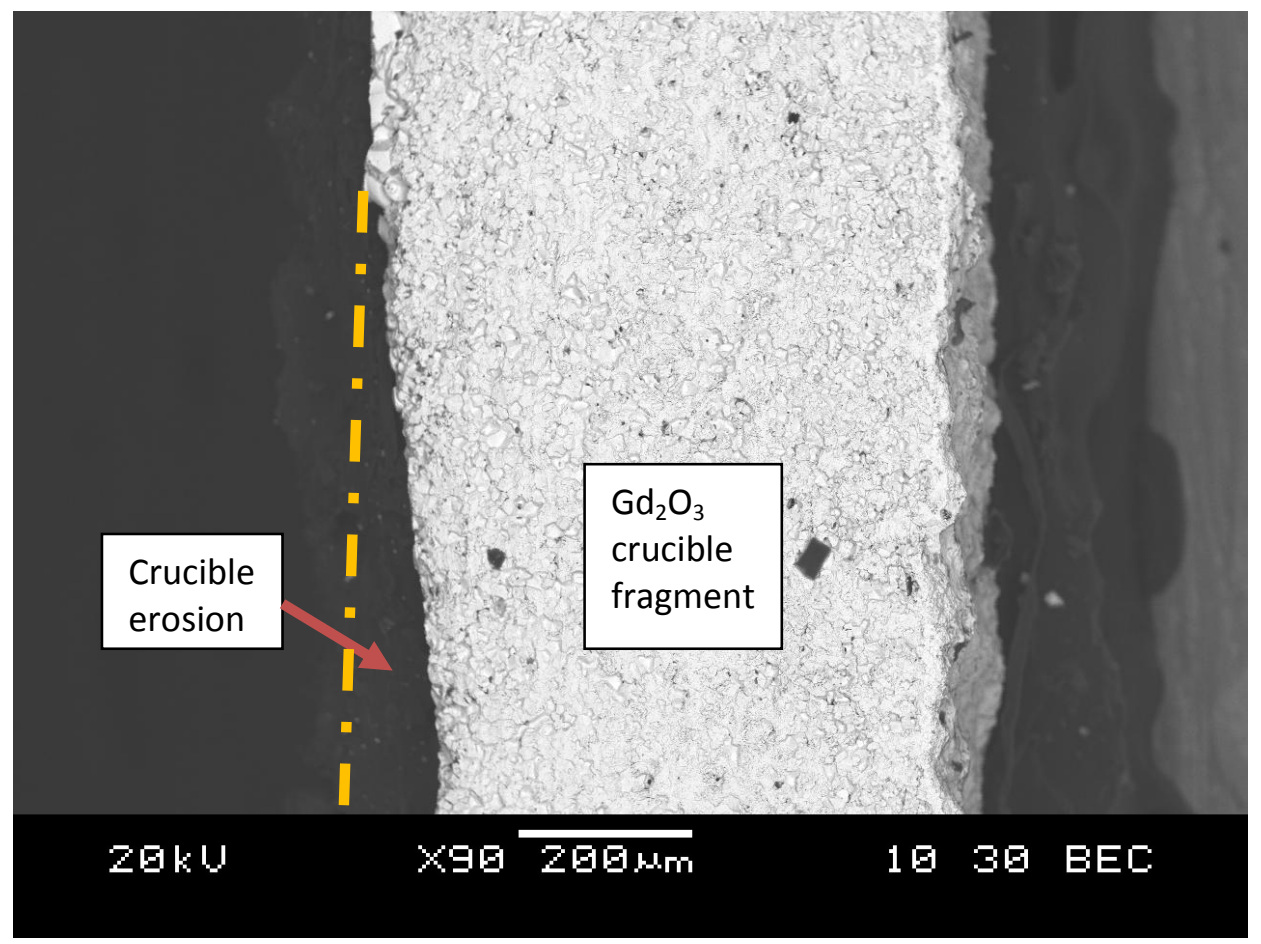

Figure 7.5: $\mathrm{A} \mathrm{Gd}_{2} \mathrm{O}_{3}$ crucible fragment from the meniscus region indicating some of the crucible has been eroded by the melt. The GdNi melt and $W$ backing have been removed. 
A sample of the GdNi was powdered and analyzed for phase purity via XRD. The scan and fit can be seen in Figure 7.6. There are no impurity phases seen, and the unit cell dimensions were measured as $a=3.7643 \pm 0.0006 \AA, b=10.3080 \pm 0.0015 \AA$, and $c=4.2423 \pm 0.0008 \AA$. The Rietveld refinement fit parameters were $R_{p}=1.62 \%, R_{w p}=2.73 \%$, $R_{\text {exp }}=0.96 \%$, and $\chi^{2}=8.09$. The lattice parameters measured are in reasonable agreement with published values considering the variation reported in crystallographic studies of GdNi, seen in Table 6.1, and are in very good agreement with the previous GdNi sample from tantalum boride coated tantalum, having deviations of less than $0.05 \%$ in each direction. The $R_{p}, R_{w p}, R_{\text {exp }}$, and $\chi^{2}$ values of the Rietveld refinement, are reasonably low, but high background is known to cause the $R$ values to be artificially low. The $\chi^{2}$ value of 8.09 is larger than the ideal value of 1 , values less than 1 are not permitted, but for phase purity analysis and general lattice parameter measurements, the fit is reasonable.

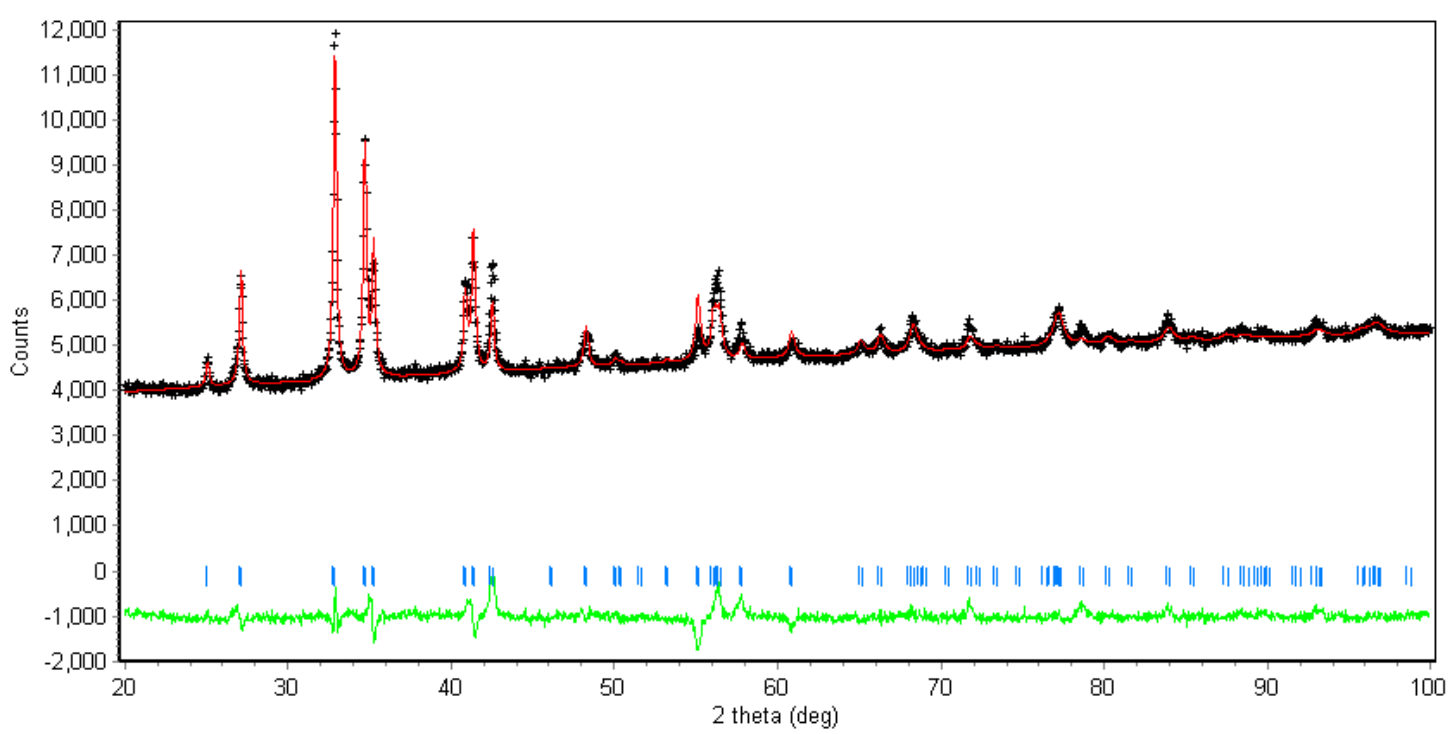

Figure 7.6: The Rietveld refinement and fit of GdNi powder from a single crystal grown in a $\mathrm{Gd}_{2} \mathrm{O}_{3}$ crucible. No impurity phases are seen. A calculated XRD pattern (red) is fitted to the data (black). The blue bars indicate where GdNi peaks are calculated to exist. The green line indicates the difference between the calculated fit and the data. 


\section{Chapter 8 Magnetic properties of GdNi}

Magnetization measurements were performed on two single crystal samples of GdNi.

One crystal was grown in a boron coated tantalum crucible and the other from an ingot grown in a plasma-sprayed $\mathrm{Gd}_{2} \mathrm{O}_{3}$ crucible. $\mathrm{M}(\mathrm{T})$ was measured on both samples, as well as $\mathrm{AC}$ susceptibility vs. temperature $(\mathrm{AC})$. The measurements were repeated on the $\mathrm{Gd}_{2} \mathrm{O}_{3}$ sample to further explore some inconsistencies seen in the boron coated tantalum data. Magnetization vs. field $(\mathrm{M}(\mathrm{H}))$ was measured only on the boron coated tantalum grown sample for there was no motivation to take the same measurements on the $\mathrm{Gd}_{2} \mathrm{O}_{3}$ grown sample since the boron coated tantalum sample appeared normal and no differences were expected to be seen.

\subsection{Magnetization vs. temperature}

The $\mathrm{M}(\mathrm{T})$ data on the $\mathrm{Gd}_{2} \mathrm{O}_{3}$ grown sample is shown in Figures 8.1-8.6, measured. All the $\mathrm{M}(\mathrm{T})$ scans are zero-field cooled $(\mathrm{ZFC})$ and heated from $2 \mathrm{~K}$ to $300 \mathrm{~K}$. $1 \mathrm{kOe}$ and $20 \mathrm{kOe} \mathrm{M}(\mathrm{T})$ scans were taken upon cooling from $300 \mathrm{~K}$ to $2 \mathrm{~K}$ to identify hysteresis. No differences between the heating and cooling curves are observed. Along the $a$ - and $c$ axes, the magnetizations in $100 \mathrm{Oe}$ and $1 \mathrm{kOe}$ fields (fig. 8.1 and 8.5) are lower at low temperatures $(\sim 2 \mathrm{~K})$ in the FM region and higher near $\mathrm{T}_{\mathrm{C}}(\sim 70 \mathrm{~K})$, but in the $b$-direction the magnetization was constant (fig. 8.3). This effect was observed to exist up to the saturating magnetic field. At these low fields, the FM to PM transition is seen as a sharp discontinuity in magnetization at $\mathrm{T}_{\mathrm{C}}$. The $100 \mathrm{Oe}$ and $1 \mathrm{kOe}$ fields indicate a $\mathrm{T}_{\mathrm{C}}$ of approximately $69-70 \mathrm{~K}$. Above the saturating field, the $\mathrm{M}(\mathrm{T})$ curves illustrate a gradual 
decline in magnetization between the FM and PM regions seen in the $20 \mathrm{kOe}$ and $40 \mathrm{kOe}$ scans of Figures 8.2, 8.4, and 8.6.

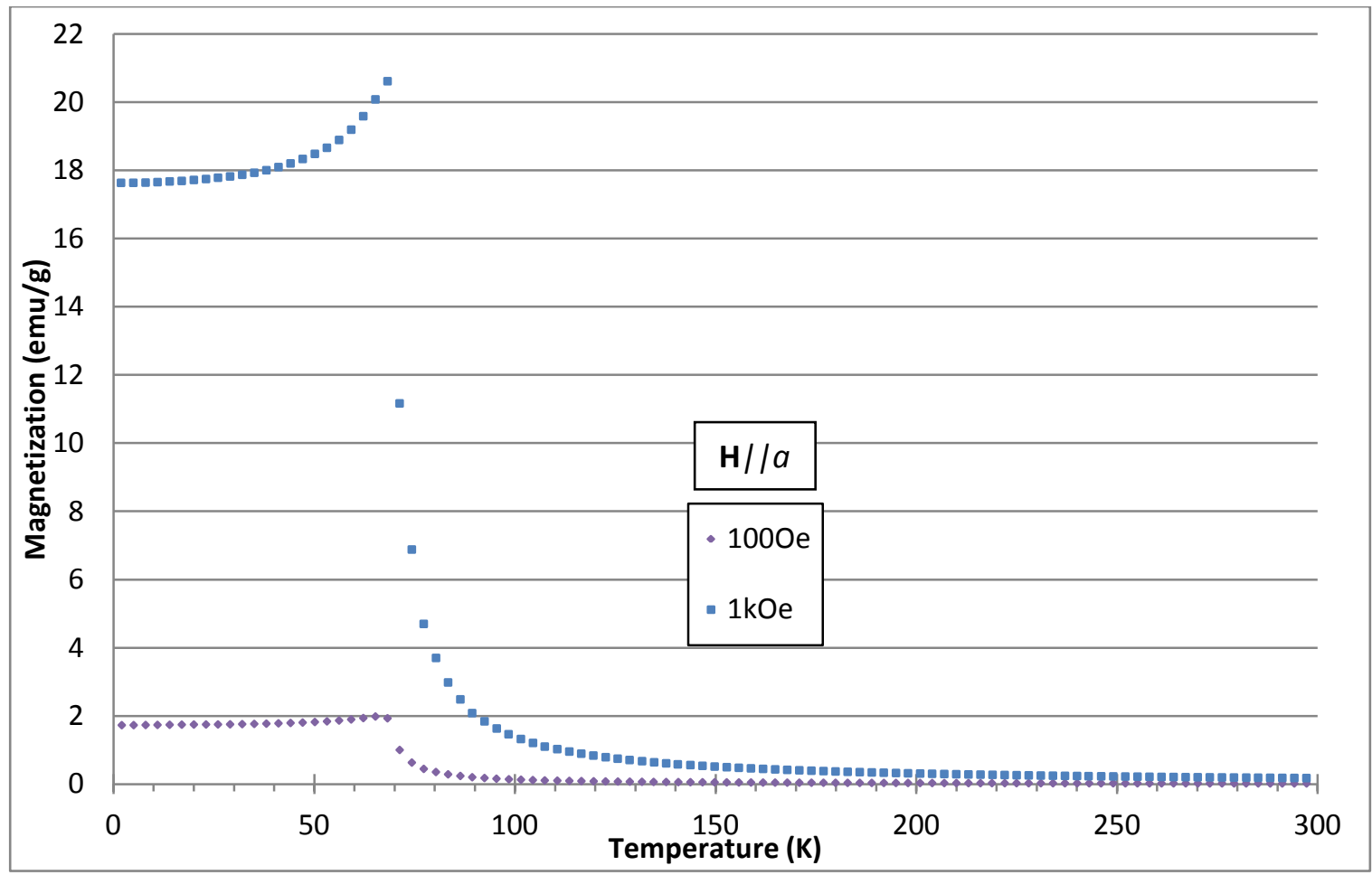

Figure 8.1: The ZFC M(T) curves in $1 \mathrm{kOe}$ and 1000 fields for single crystal GdNi, $\mathrm{H} \| a$. 


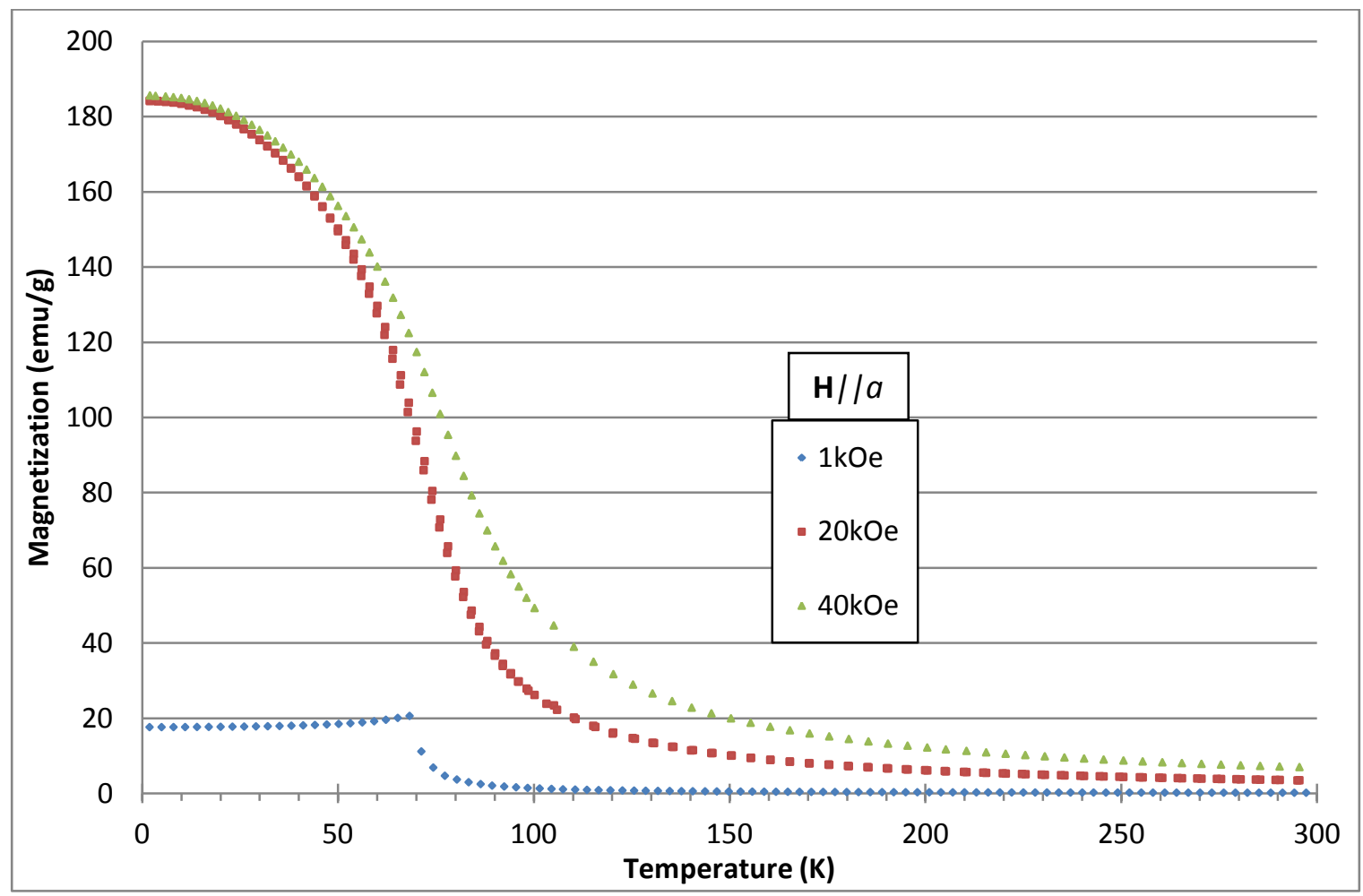

Figure 8.2: The ZFC M(T) curves in $1 \mathrm{kOe}, 20 \mathrm{kOe}$ and $40 \mathrm{kOe}$ fields for single crystal GdNi, $\mathrm{H} \|$ a.

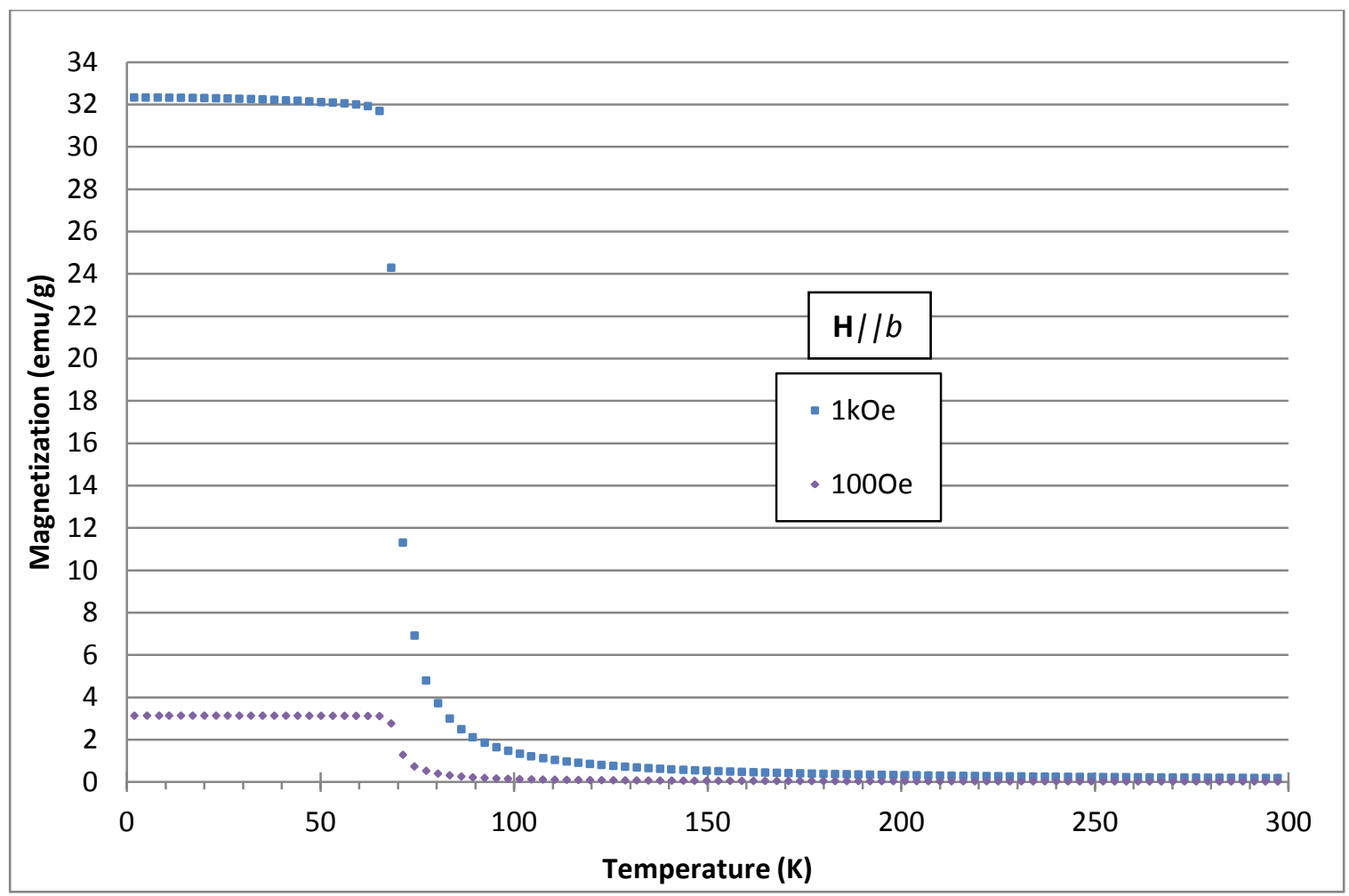

Figure 8.3: The ZFC M(T) curves in 1kOe and 100Oe fields for single crystal GdNi, $H \| b$. 


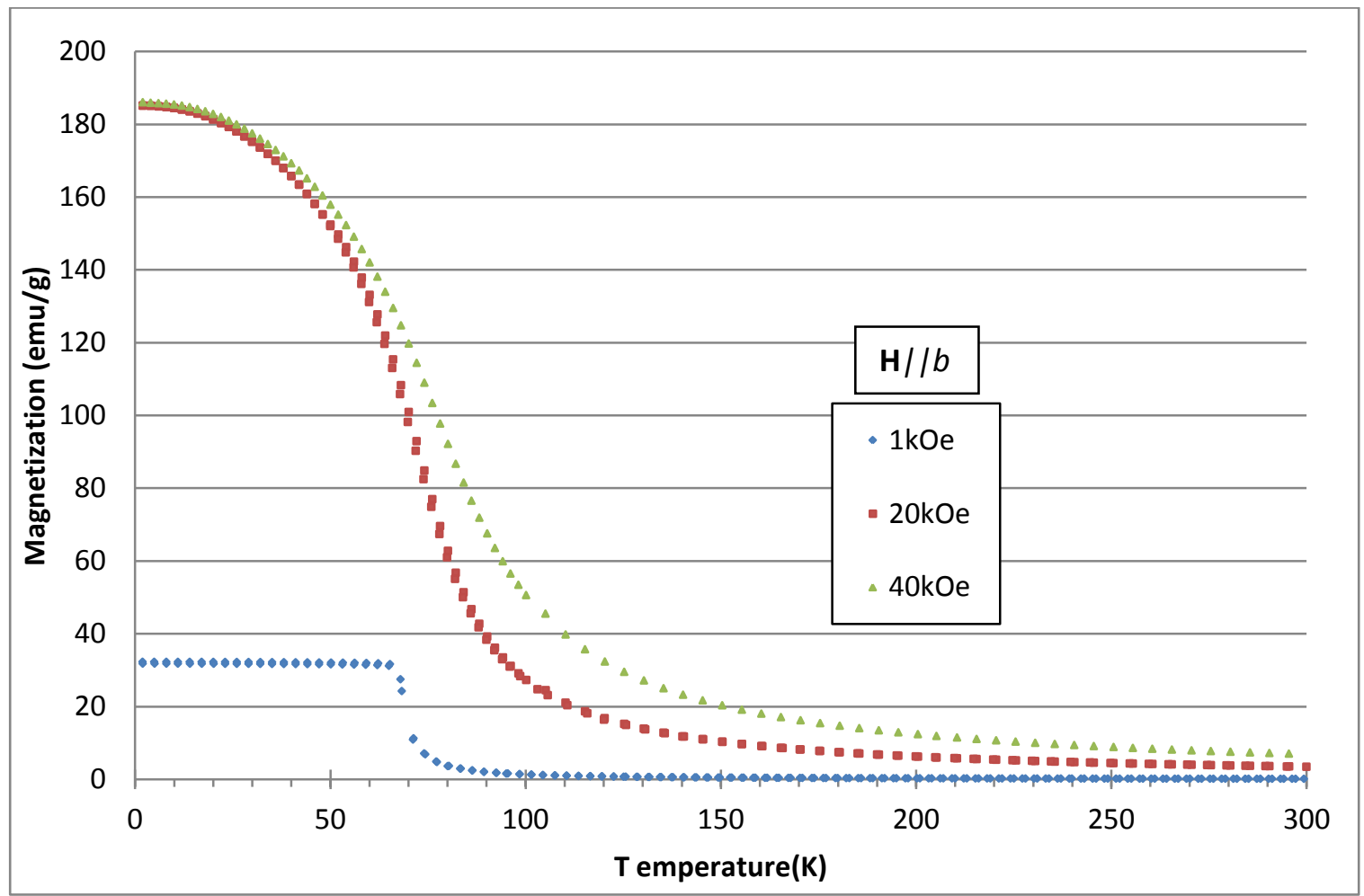

Figure 8.4: The ZFC M(T) curves in $1 \mathrm{kOe}, 20 \mathrm{kOe}$ and 40kOe fields for single crystal GdNi, $\mathrm{H} \| b$.

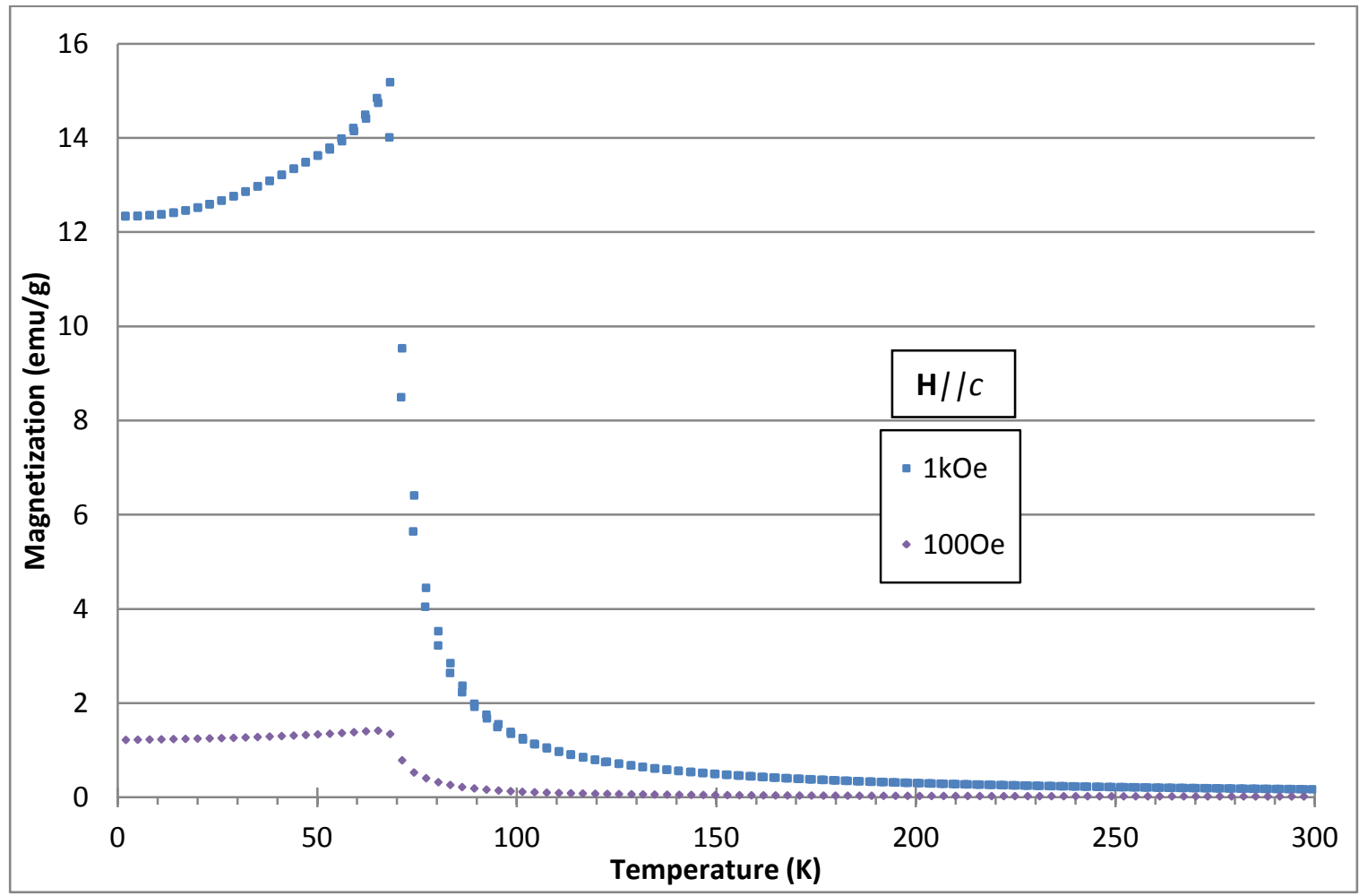

Figure 8.5: The ZFC M(T) curves in 1kOe and 1000e fields for single crystal GdNi, $\mathrm{H} \|$. 


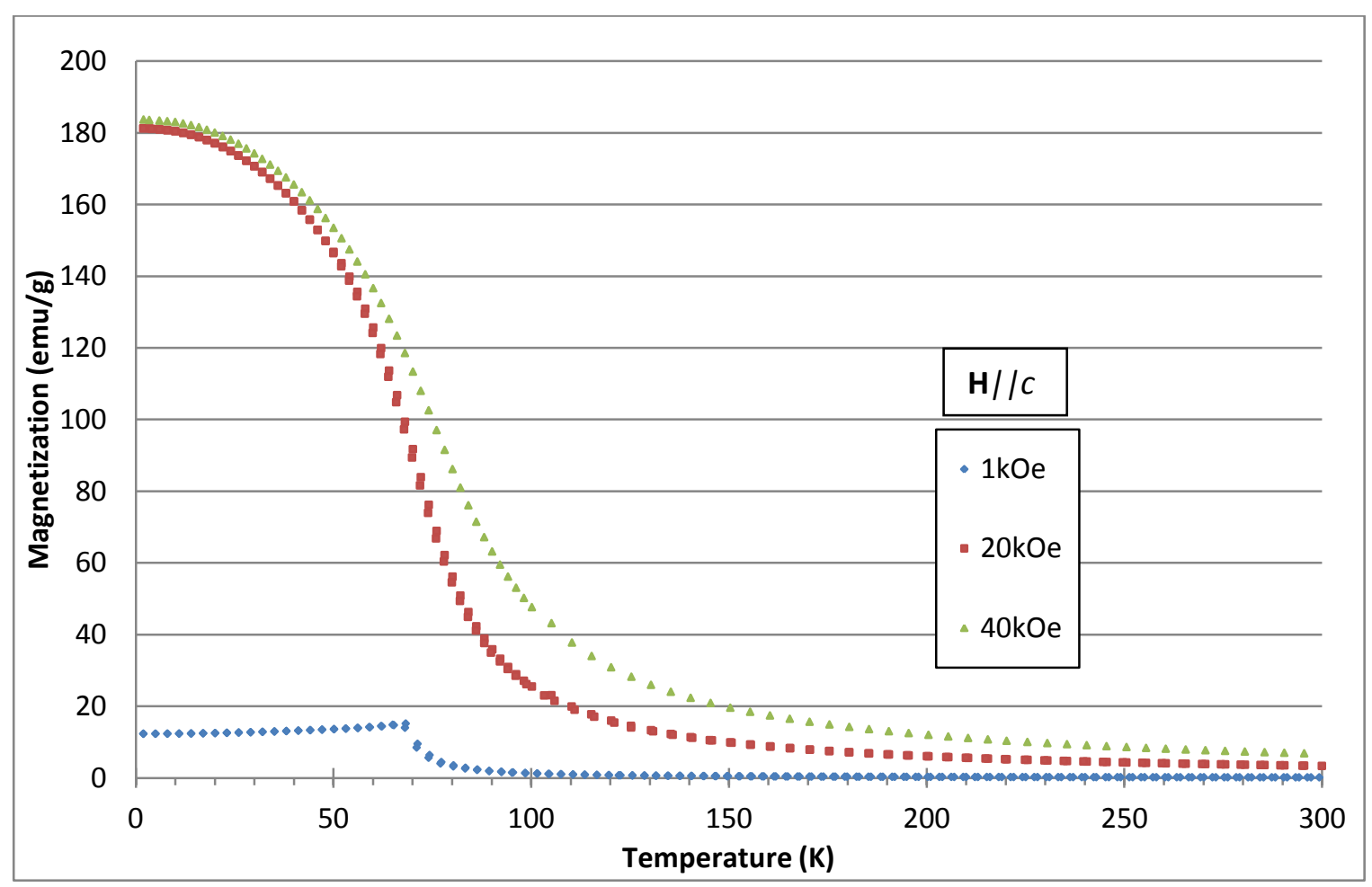

Figure 8.6: The ZFC M(T) curves in $1 \mathrm{kOe}, 20 \mathrm{kOe}$ and $40 \mathrm{kOe}$ fields for single crystal GdNi, $\mathrm{H} \| c$.

\subsection{Inverse magnetic susceptibility}

The inverse magnetic susceptibility curves $(\mathrm{H} / \mathrm{M}(\mathrm{T}))$ for GdNi were calculated from the $\mathrm{M}(\mathrm{T})$ data. Data from both the coated tantalum and $\mathrm{Gd}_{2} \mathrm{O}_{3}$ grown samples are compared in Figures 8.7 and 8.8 (a-axis), 8.9 and 8.10 (b-axis), and 8.11 and 8.12 (c-axis). The coated tantalum grown sample data is not as linear in the paramagnetic region where the Curie-Weiss law is in effect and the $\mathrm{Gd}_{2} \mathrm{O}_{3}$ grown sample has a much better slope agreement between fields in this region. The reason for the deviation in slopes is thought to be due to pure Gd ferromagnetic impurity, which saturates and has little effect at high fields, but at low fields, such as 1000 e, the inverse paramagnetic susceptibility is skewed to have a slightly greater slope. The $\mathrm{Gd}_{2} \mathrm{O}_{3}$ grown sample is a better crystal with fewer 
impurities as indicated by this measurement. Slopes from these susceptibility data were used to calculate the paramagnetic Weiss temperatures $\left(\theta_{\mathrm{p}}\right)$ and effective moments ( $\left.\mathrm{p}_{\text {eff }}\right)$ which were slightly anisotropic. The calculated Weiss temperatures and $p_{\text {eff }}$ values are listed in Table 8.1. The theoretical value of $\mathrm{p}_{\text {eff }}$ for non-interacting magnetic ions is $p_{\text {eff }}=7.94 \mu_{\mathrm{B}}$ calculated from the quantum numbers of Gd, shown in equation 1. Slightly higher values are expected from the interaction of conduction electrons and polarization of the $5 d$ electrons.

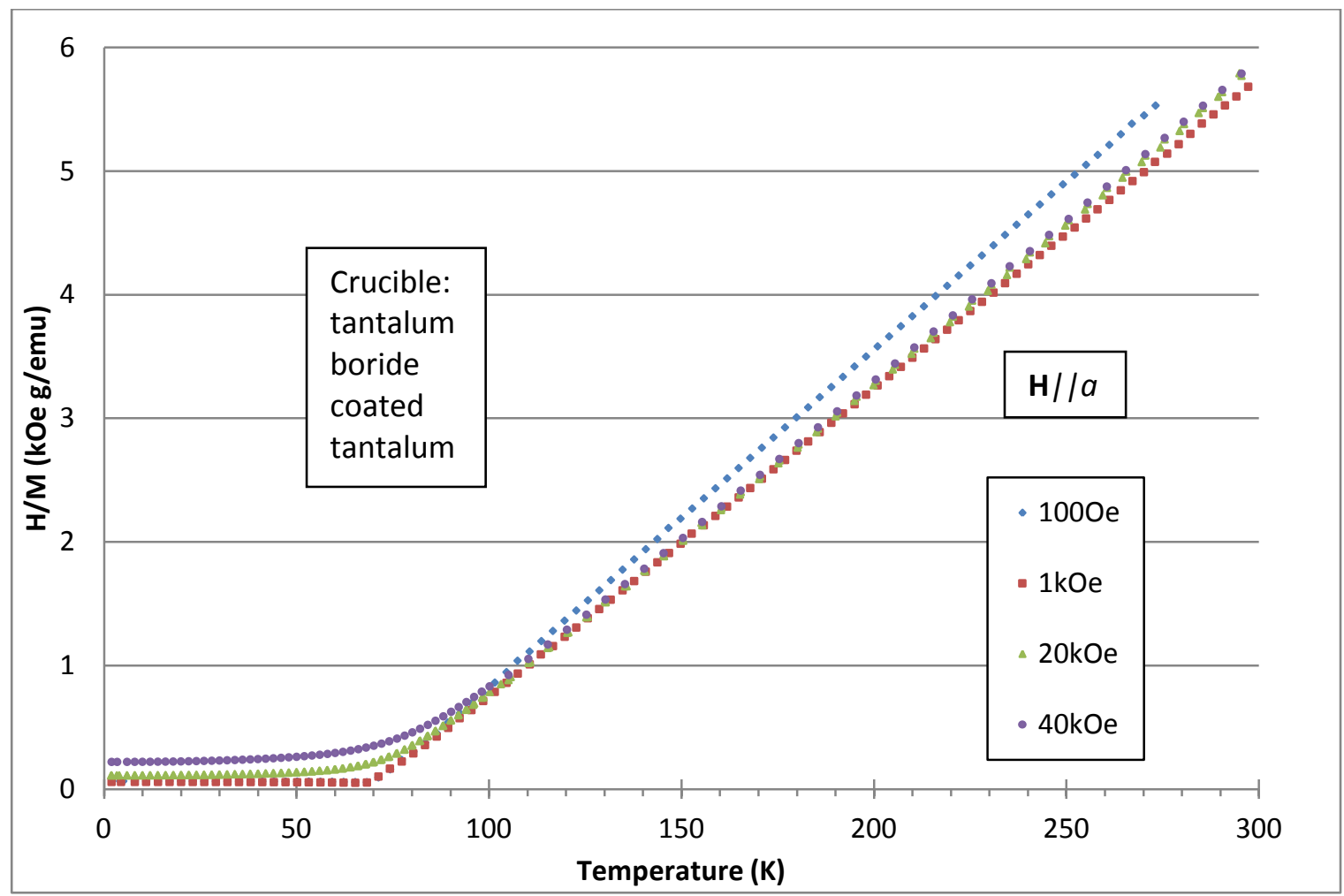

Figure 8.7: Inverse magnetic susceptibility for single crystal GdNi grown in tantalum boride coated tantalum, $\mathbf{H} \|$. 


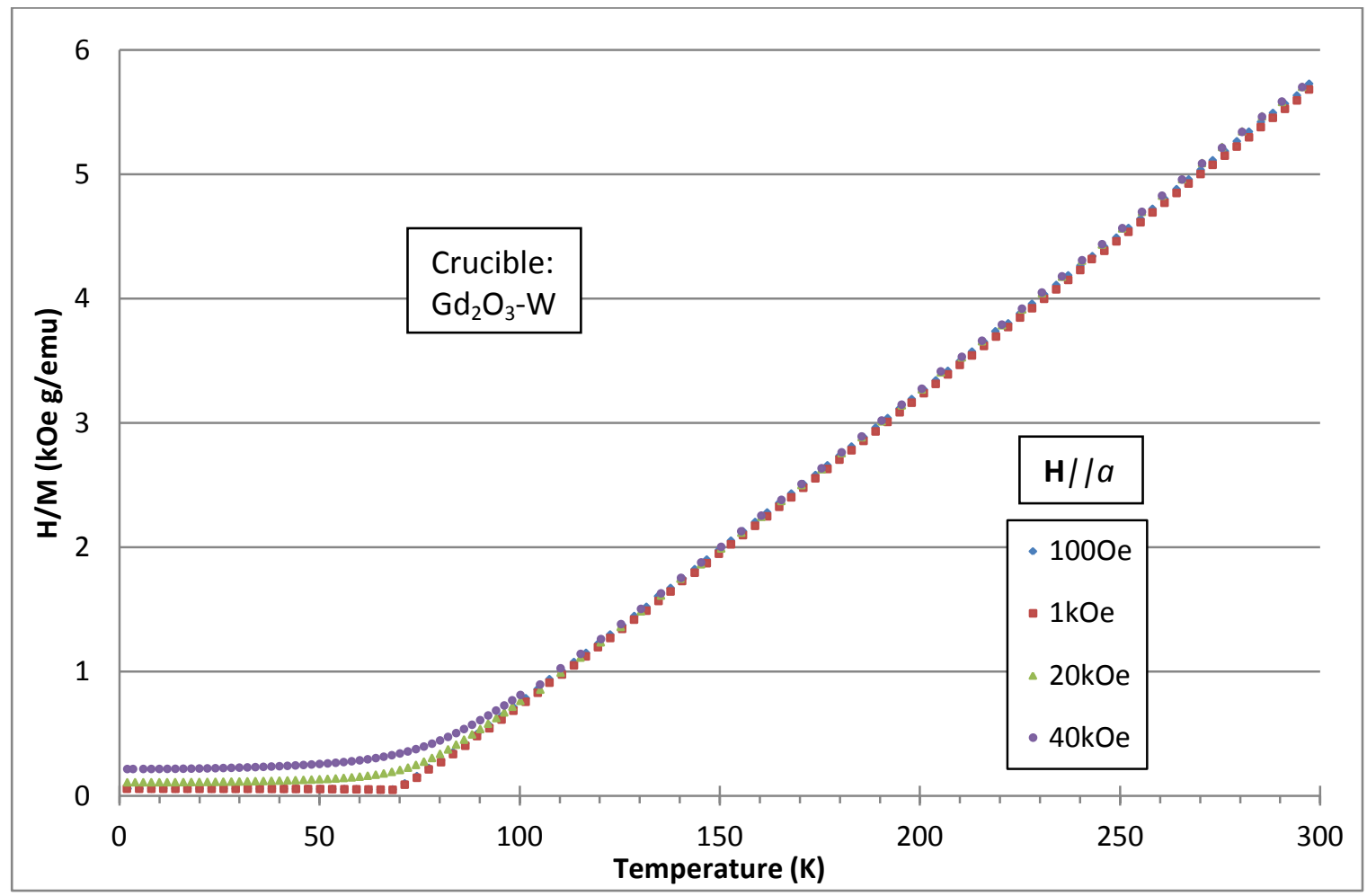

Figure 8.8: Inverse magnetic susceptibility for single crystal GdNi grown in $\mathrm{Gd}_{2} \mathrm{O}_{3}, \mathrm{H} \| a$.

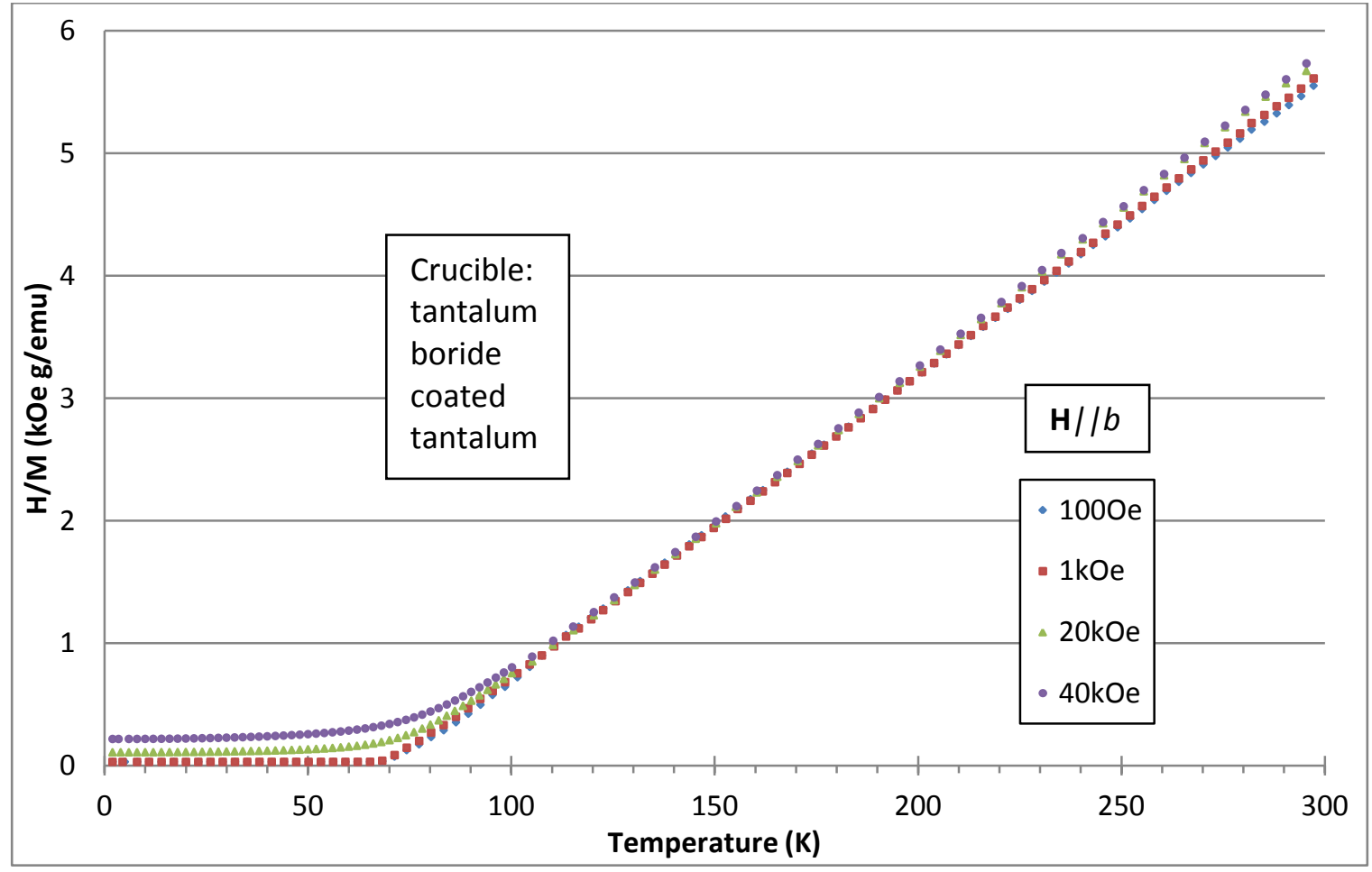

Figure 8.9: Inverse magnetic susceptibility for single crystal GdNi grown in tantalum boride coated tantalum, $\mathbf{H} \|$. 


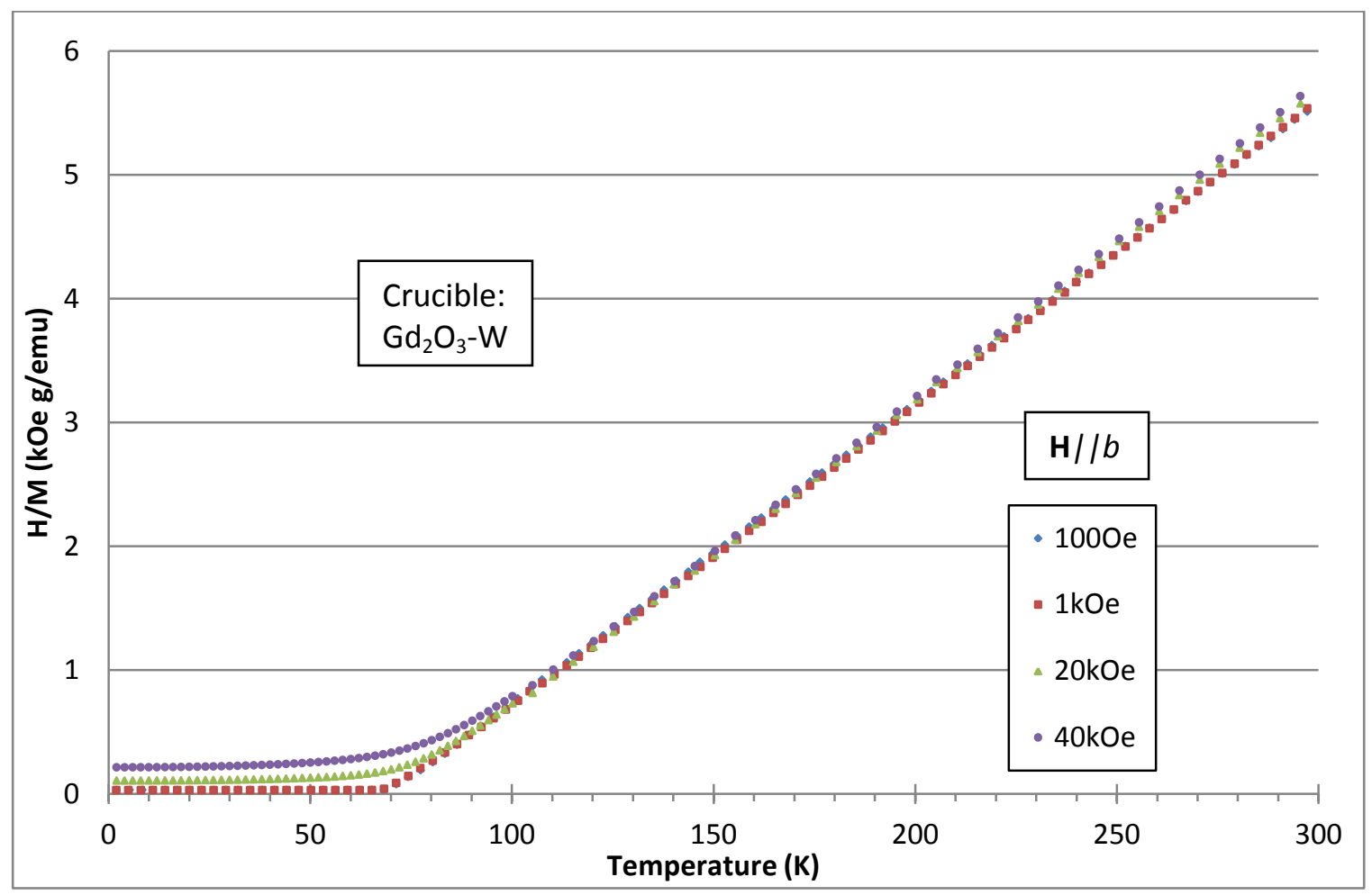

Figure 8.10: Inverse magnetic susceptibility for single crystal GdNi grown in $\mathrm{Gd}_{2} \mathrm{O}_{3}, \mathrm{H} \|$.

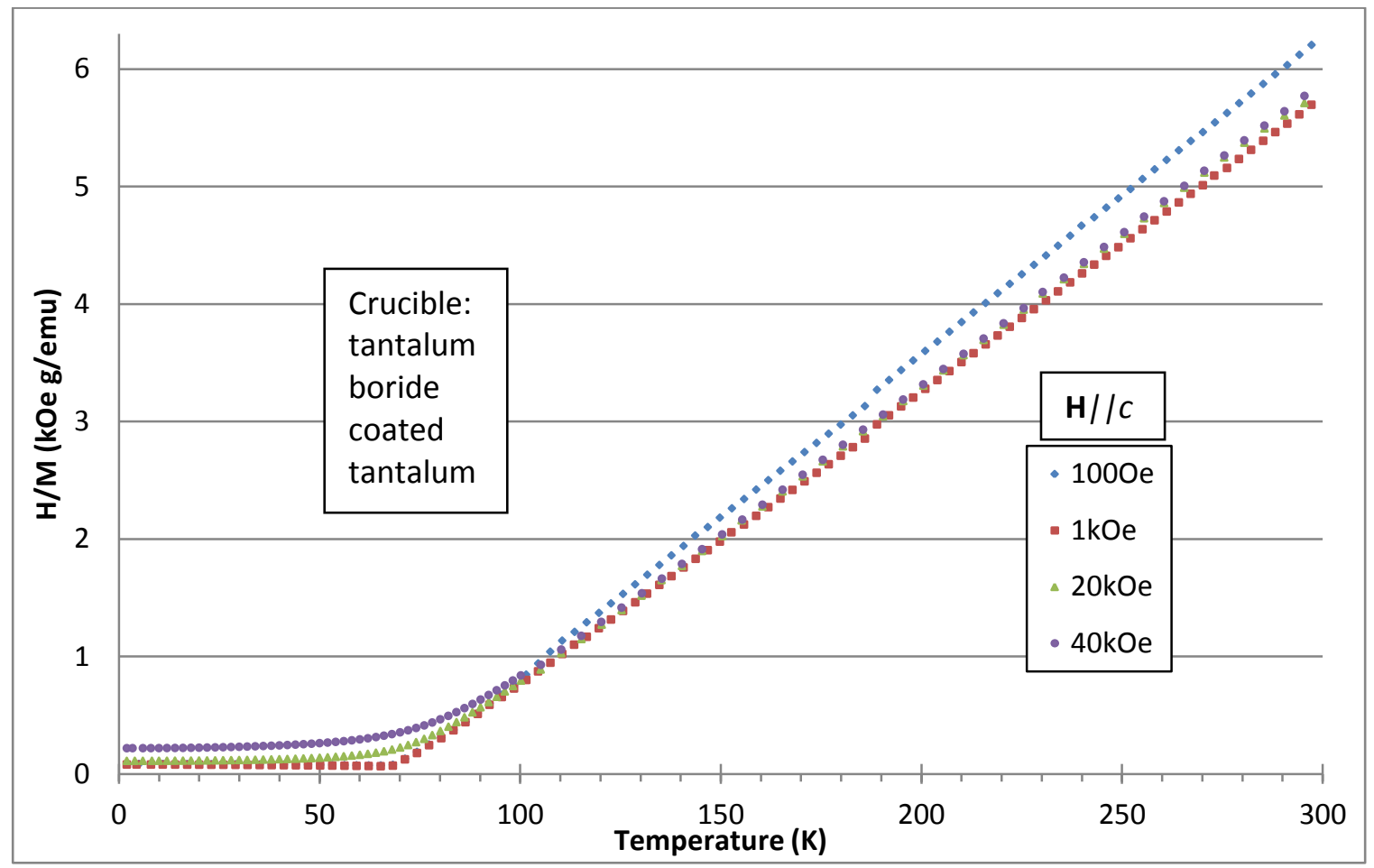

Figure 8.11: Inverse magnetic susceptibility for single crystal GdNi grown in tantalum boride coated tantalum, $\mathbf{H} \| c$. 


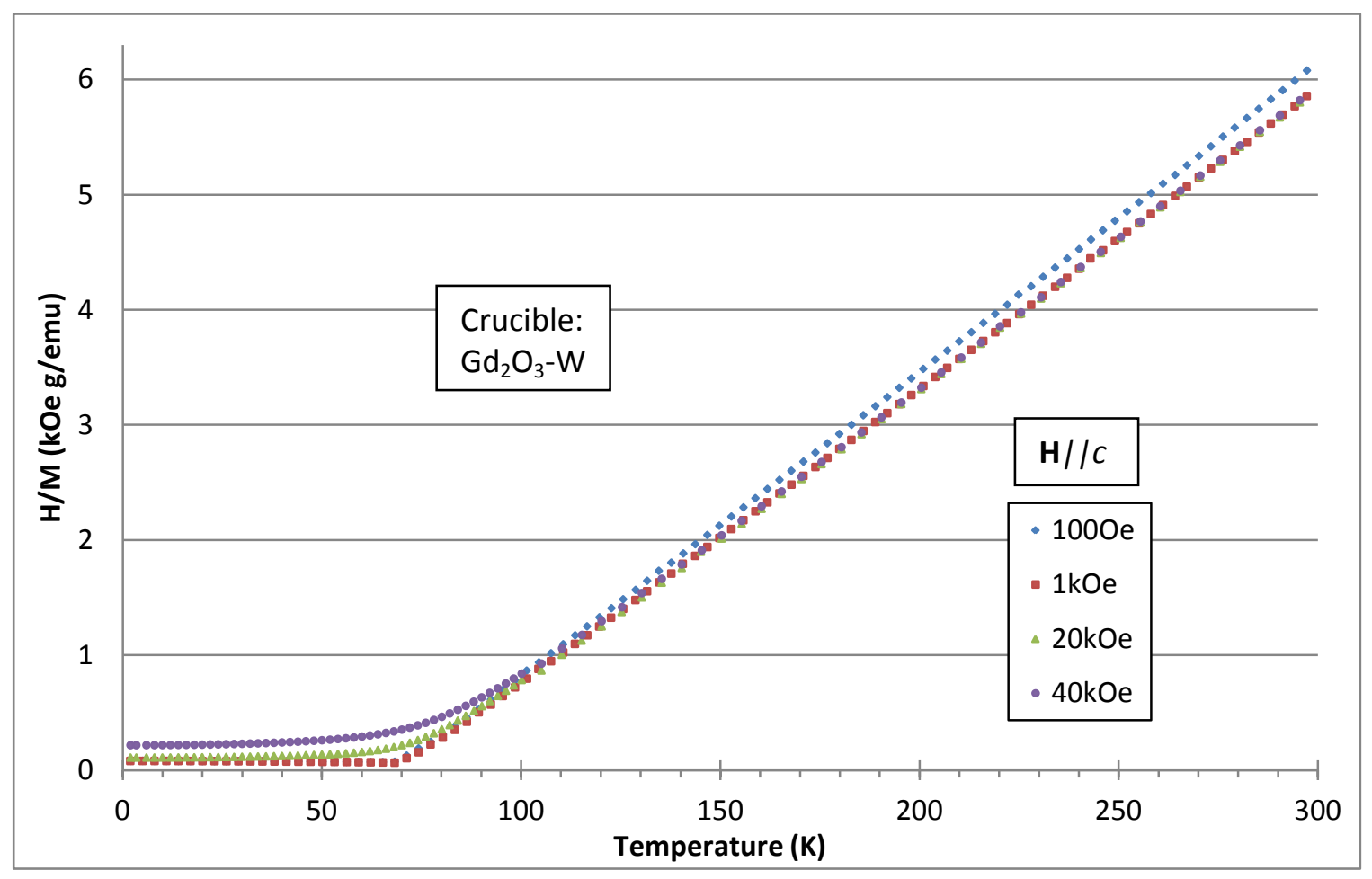

Figure 8.12: Inverse magnetic susceptibility for single crystal GdNi grown in $\mathrm{Gd}_{2} \mathrm{O}_{3}, \mathrm{H} \| c$.

Table 8.1: The Weiss temperatures and effective moments calculated from the $40 \mathrm{kOe} \mathrm{H} / \mathrm{M}(\mathrm{T})$ data from the $\mathrm{Gd}_{2} \mathrm{O}_{3}$ grown single crystal.

\begin{tabular}{|l|c|c|}
\hline & $\theta \mathrm{p}(\mathrm{K})$ & $\mathrm{P}_{\text {eff }}(\mu \mathrm{B} / \mathrm{f} . \mathrm{u})$. \\
\hline$a$-axis & $72.1 \pm 0.4$ & $8.212 \pm 0.014$ \\
\hline$b$-axis & $72.4 \pm 0.4$ & $8.277 \pm 0.014$ \\
\hline$c$-axis & $71.7 \pm 0.5$ & $8.162 \pm 0.014$ \\
\hline
\end{tabular}

\subsection{AC susceptibility}

In an effort to further investigate the phase purity of GdNi crystals AC susceptibility measurements were taken. The AC susceptibility measurement was chosen because it is particularly sensitive to changes in magnetization. The AC susceptibility curves from the $\mathrm{Gd}_{2} \mathrm{O}_{3}$ grown sample are shown in Figures 8.13-8.18 for both the real, $\chi^{\prime}(\mathrm{T})$, and imaginary, $\chi$ ”(T), components. All AC susceptibility measurements were made in a 5Oe amplitude field at a $100 \mathrm{~Hz}$ frequency. 


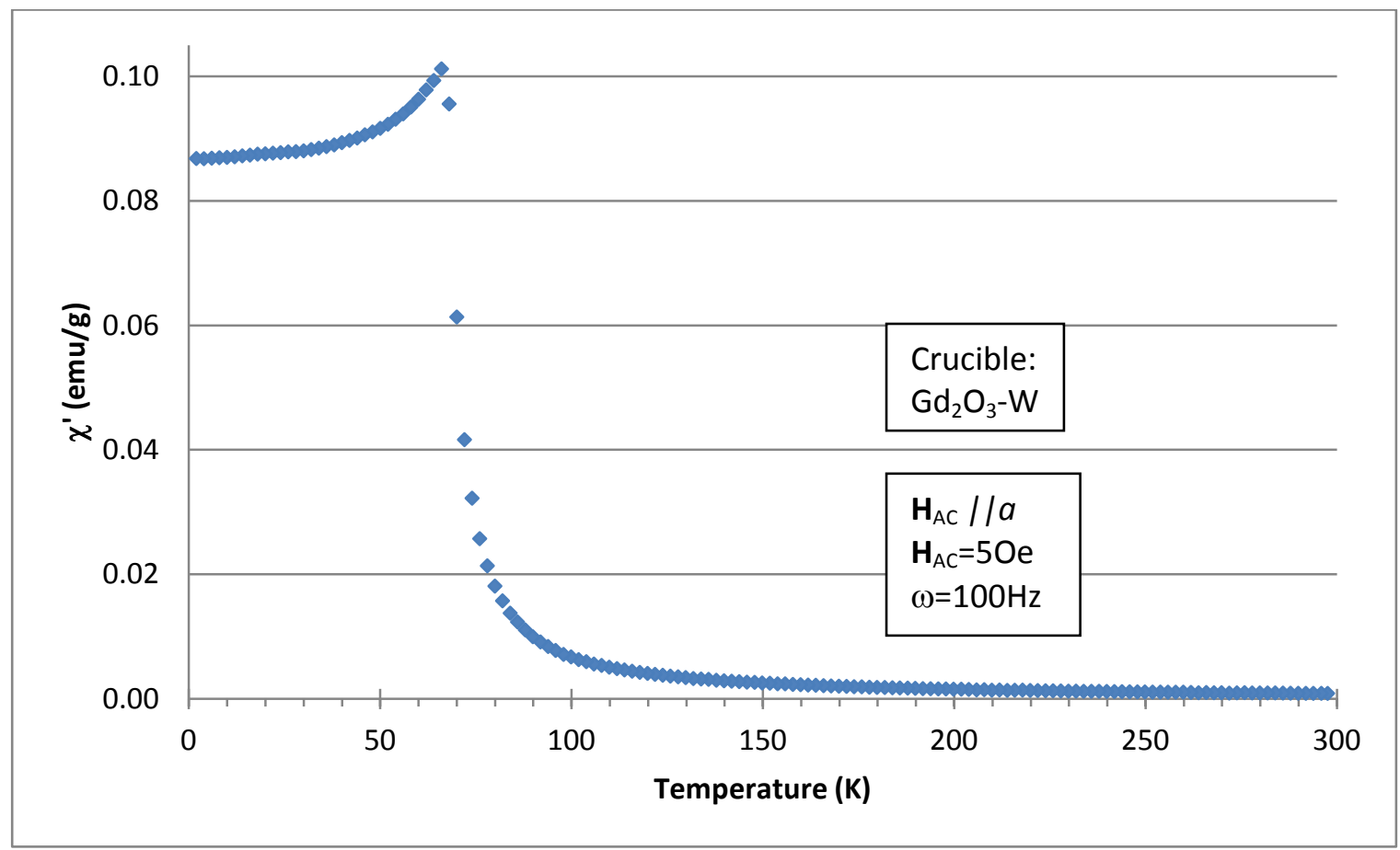

Figure 8.13: Real component of AC susceptibility measured along the $a$-axis of single crystal GdNi grown in $\mathbf{G d}_{2} \mathbf{O}_{3}$.

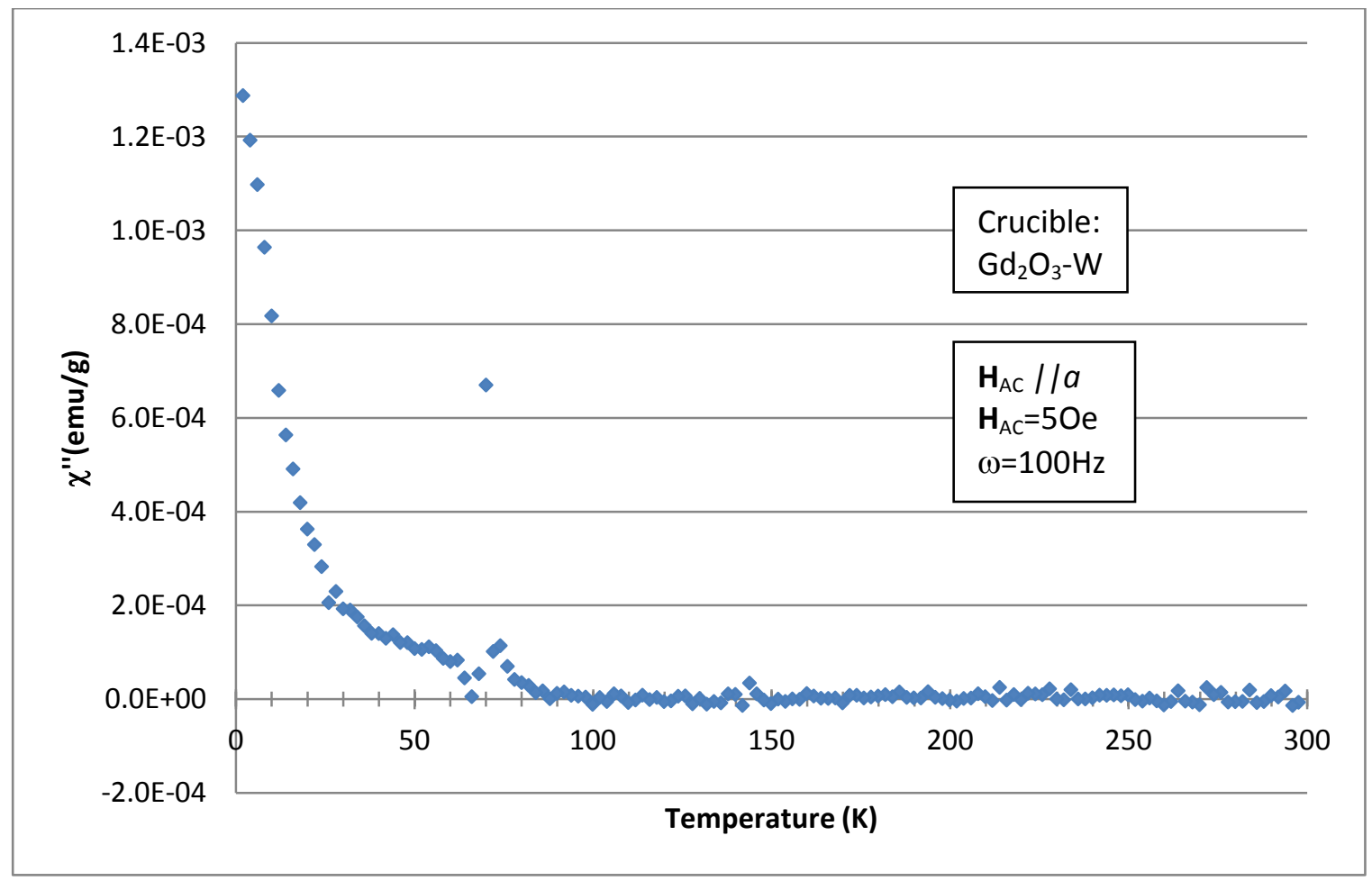

Figure 8.14: Imaginary component of AC susceptibility measured along the $a$-axis of single crystal GdNi grown in $\mathrm{Gd}_{2} \mathrm{O}_{3}$. 


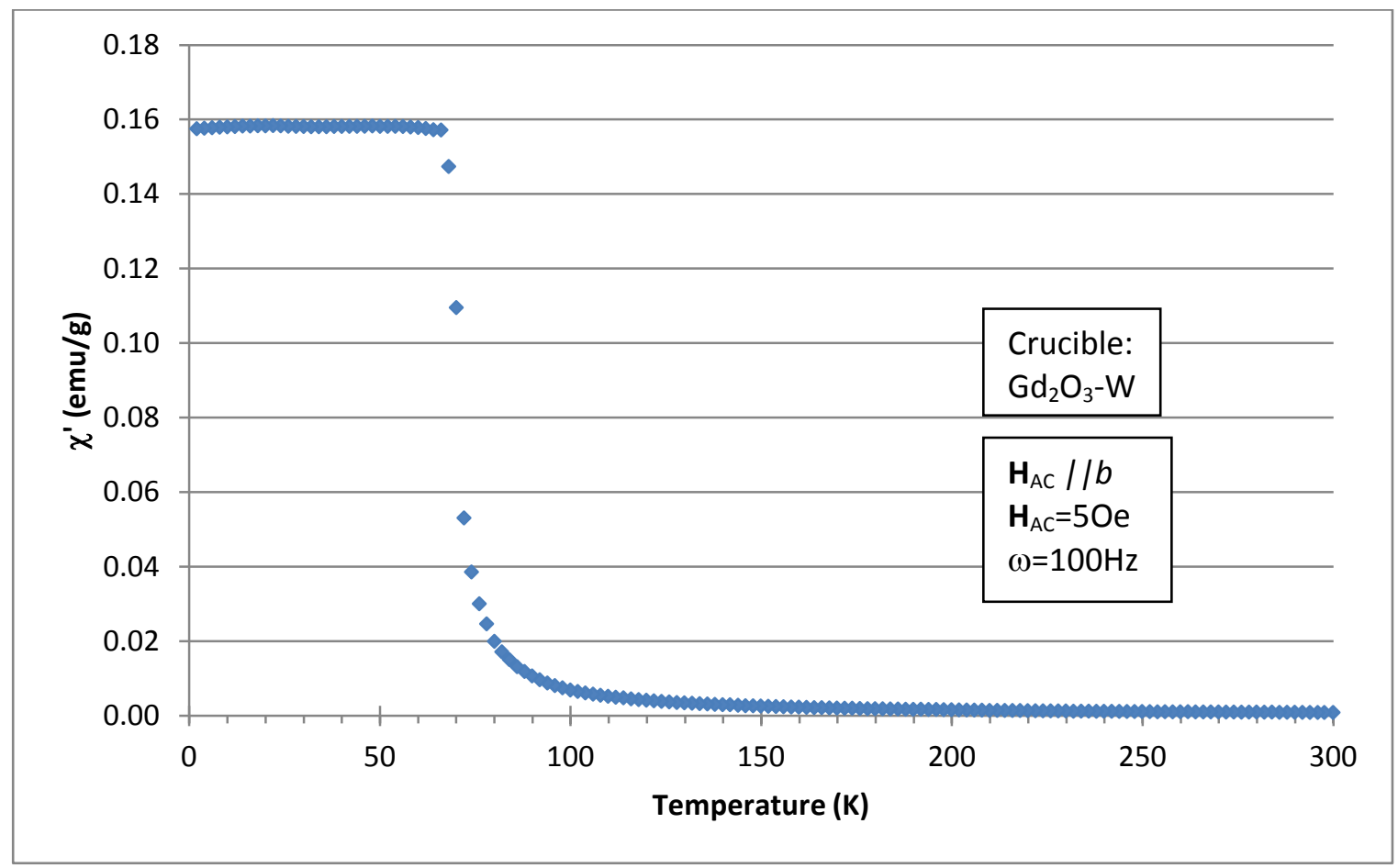

Figure 8.15: Real component of AC susceptibility measured along the $b$-axis of single crystal GdNi grown in $\mathrm{Gd}_{2} \mathrm{O}_{3}$.

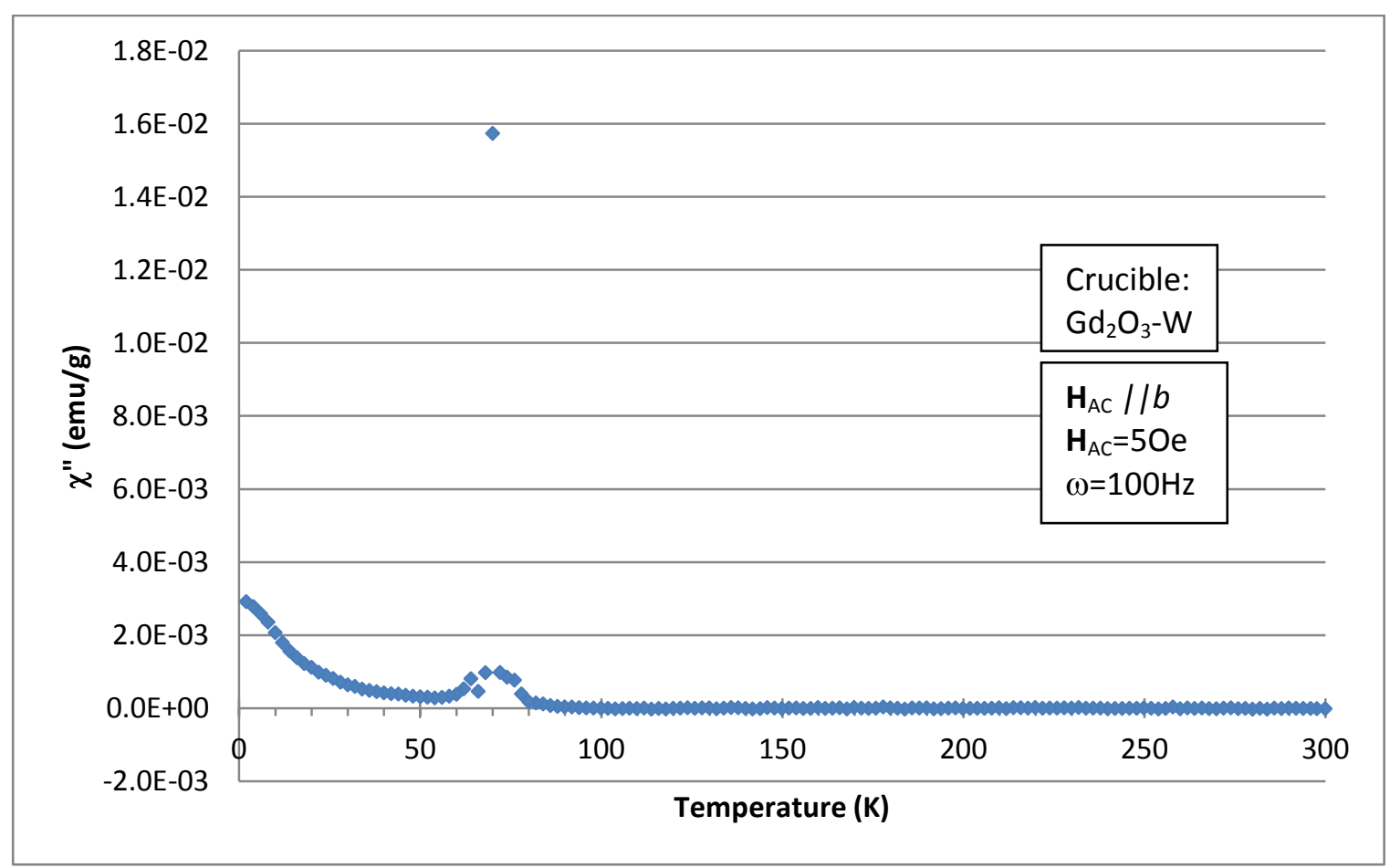

Figure 8.16: Imaginary component of $\mathrm{AC}$ susceptibility measured along the $b$-axis of single crystal GdNi grown in $\mathrm{Gd}_{2} \mathrm{O}_{3}$. 


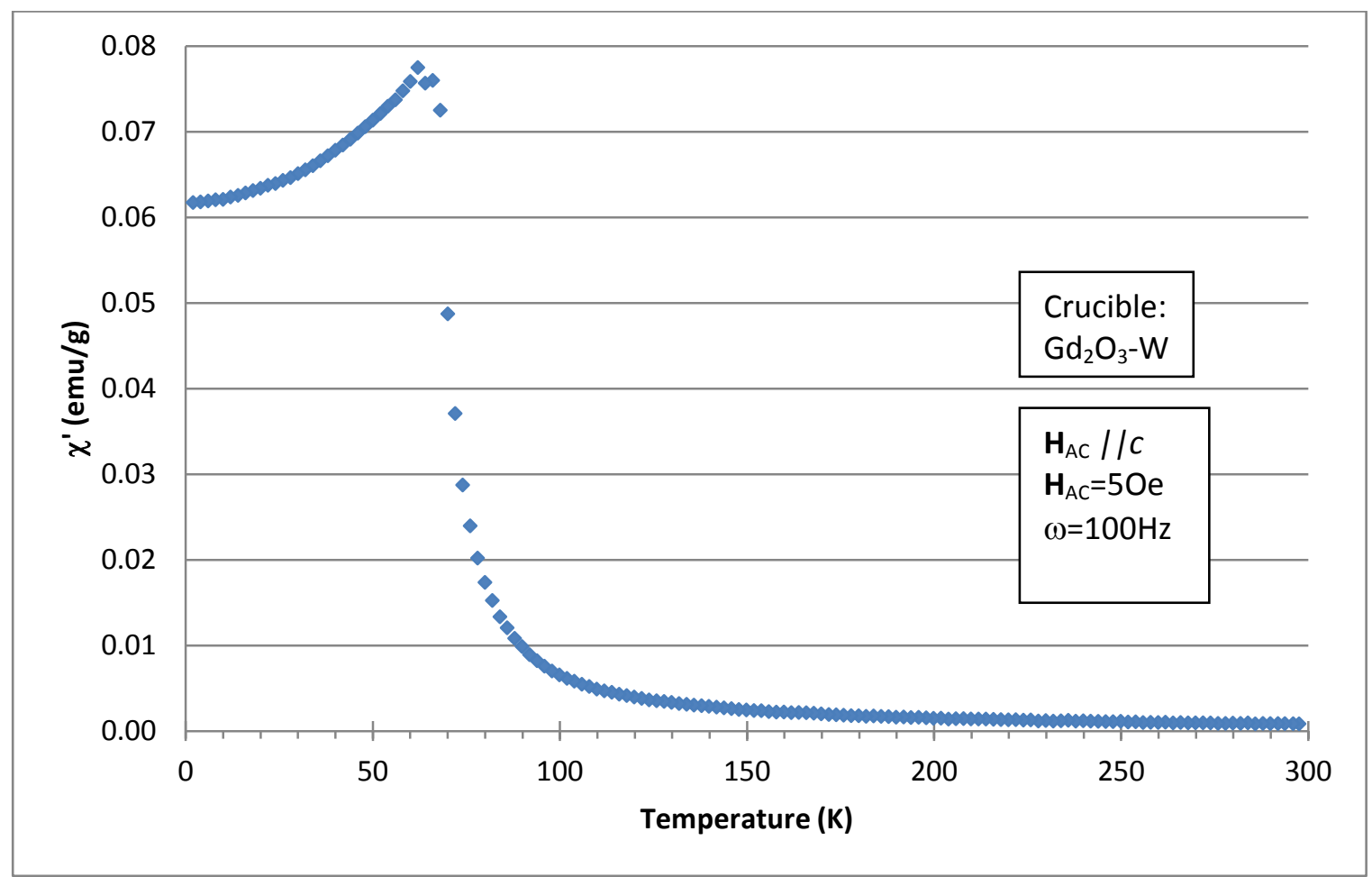

Figure 8.17: Real component of AC susceptibility measured along the $c$-axis of single crystal GdNi grown in $\mathbf{G d}_{2} \mathbf{O}_{3}$.

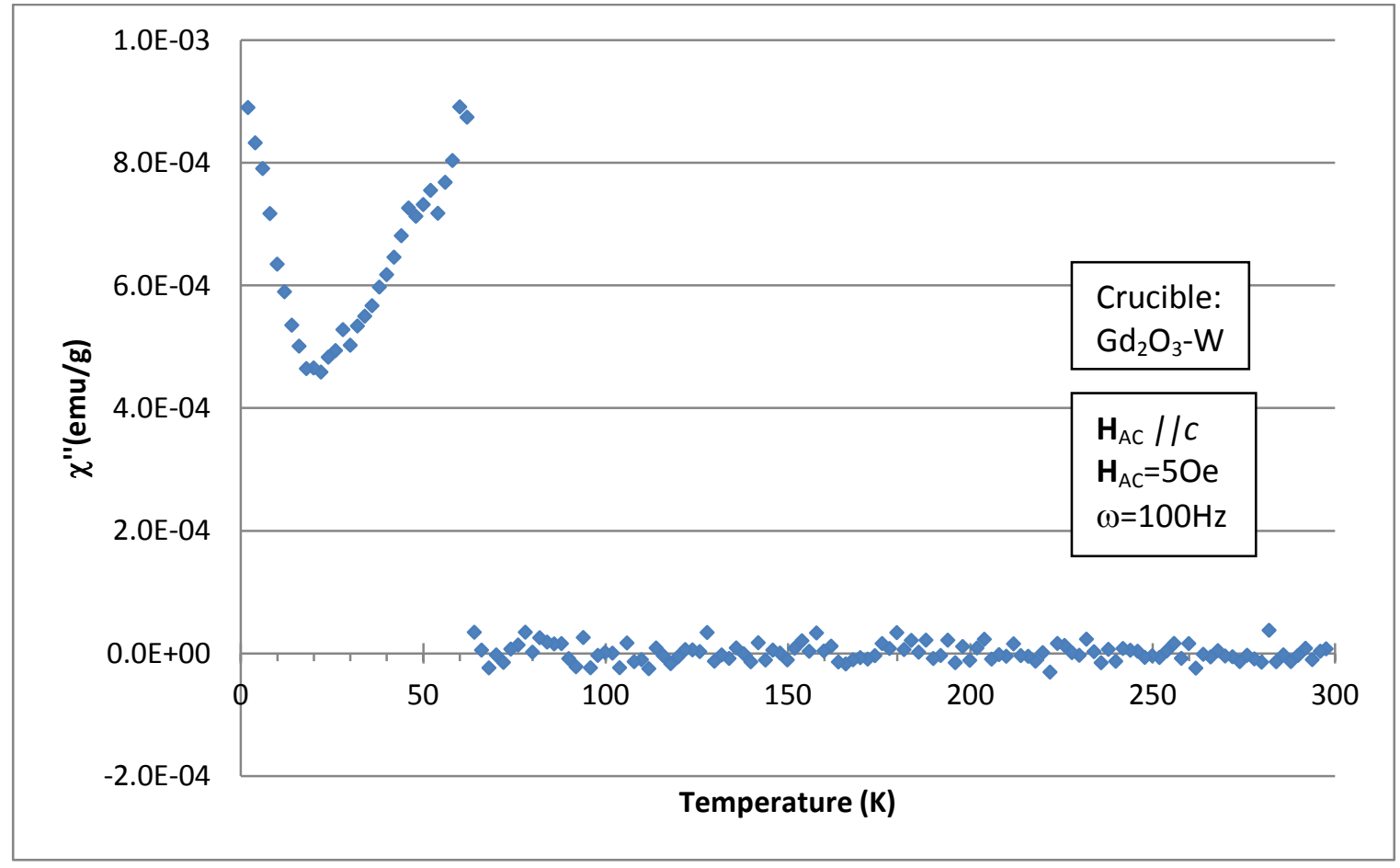

Figure 8.18: Imaginary component of $\mathrm{AC}$ susceptibility measured along the $c$-axis of single crystal GdNi grown in $\mathrm{Gd}_{2} \mathrm{O}_{3}$. 
With the added sensitivity, an impurity phase was detected in the boron coated tantalum sample, which was not seen in other characterizations. In the boron coated tantalum grown sample, an anomaly is seen only along the $b$-axis in Figure 8.19 and 8.20. An additional transition around $100 \mathrm{~K}$ is attributed to $\mathrm{Gd}_{3} \mathrm{Ni}$ impurity but the transition is not seen in the same sample along the other two axes. The $\mathrm{Gd}_{2} \mathrm{O}_{3}$ grown sample does not have this transition in the $b$-axis measurement. The boron coated tantalum $a$-axis and $c$ axis AC data sets (not included) are nearly identical to the equivalent measurements of the $\mathrm{Gd}_{2} \mathrm{O}_{3}$ grown sample, Figures $8.13,8.14,8.17$, and 8.18 .

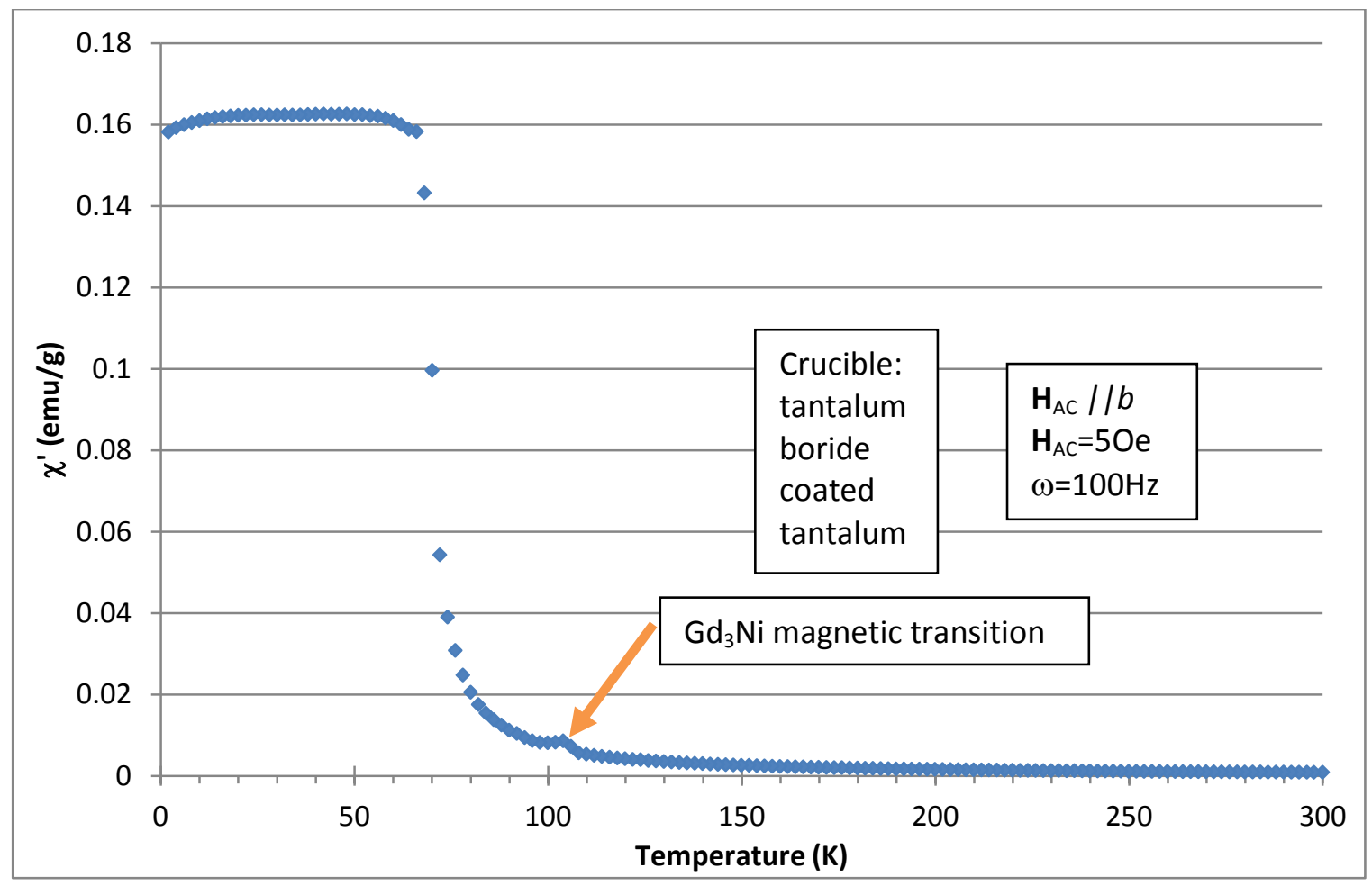

Figure 8.19: Real component of AC susceptibility measured along the $b$-axis of single crystal GdNi grown in tantalum boride coated tantalum. 


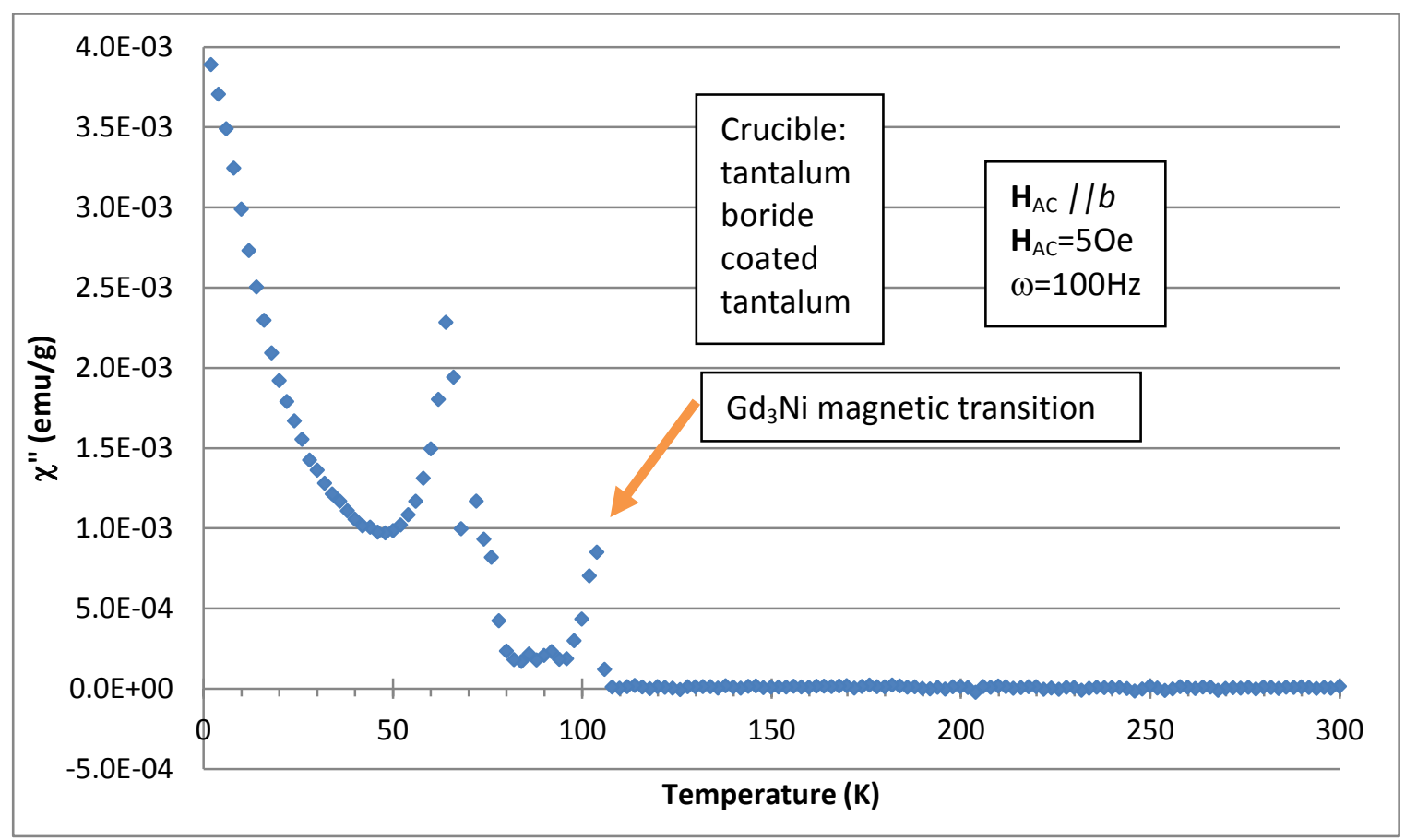

Figure 8.20: Imaginary component of AC susceptibility measured along the $b$-axis of single crystal GdNi grown in tantalum boride coated tantalum.

\subsection{Isothermal magnetization}

GdNi has a measured saturated magnetic moment of $7.10 \pm 0.03 \mu_{\mathrm{B}} /$ f.u. along the $a$-axis, $7.17 \pm 0.03 \mu_{\mathrm{B}} /$ f.u. along the $b$-axis, and $7.12 \pm 0.03 \mu_{\mathrm{B}} /$ f.u. along the $c$-axis. This is slightly higher than the theoretical value of the non-interacting $\mathrm{Gd}^{3+}$ moment of $7 \mu_{\mathrm{B}}$ but this is in reasonable agreement with the theoretically calculated from first principals value of $7.20 \mu_{\mathrm{B}} /$ f.u. from Paudyal et al. which accounts for additional electron interactions. ${ }^{17}$ However, we don’t expect to see a slight anisotropy along the crystallographic axes. In Figure 8.26 the $\mathrm{M}(\mathrm{H})$ curves at $2 \mathrm{~K}$ can be seen for the $a, b$, and $c$ directions and they saturate at roughly $10 \mathrm{kOe}, 4 \mathrm{kOe}$ and $13 \mathrm{kOe}$, respectively. The $\mathrm{M}(\mathrm{H})$ data has been corrected with a demagnetizing factor determined from Chen et al. ${ }^{40}$ The full magnetization loops were measured at $2 \mathrm{~K}$ in sequence from $0 \mathrm{Oe}$ to $70 \mathrm{kOe}$ to $-70 \mathrm{kOe}$ to 70kOe. The loops show almost zero hysteresis, GdNi is a very soft magnet with a 
coercivity of approximately 5-10Oe in each direction. The $\mathrm{M}(\mathrm{H})$ curves for higher temperatures, 5-100K, are shown in Figures 8.22-8.24. The $5 \mathrm{~K}$ and $50 \mathrm{~K}$ data sets are collected with a finer field increment to reveal the difference in magnetization in the ferromagnetic region, especially in the $a$ and $c$ directions. In each of the higher temperature $\mathrm{M}(\mathrm{H})$ curves, the $60 \mathrm{~K}$ magnetization behaves ferromagnetically and the $70 \mathrm{~K}$ magnetization behaves paramagnetically, agreeing with a $T_{C}$ between $60-70 \mathrm{~K}$.

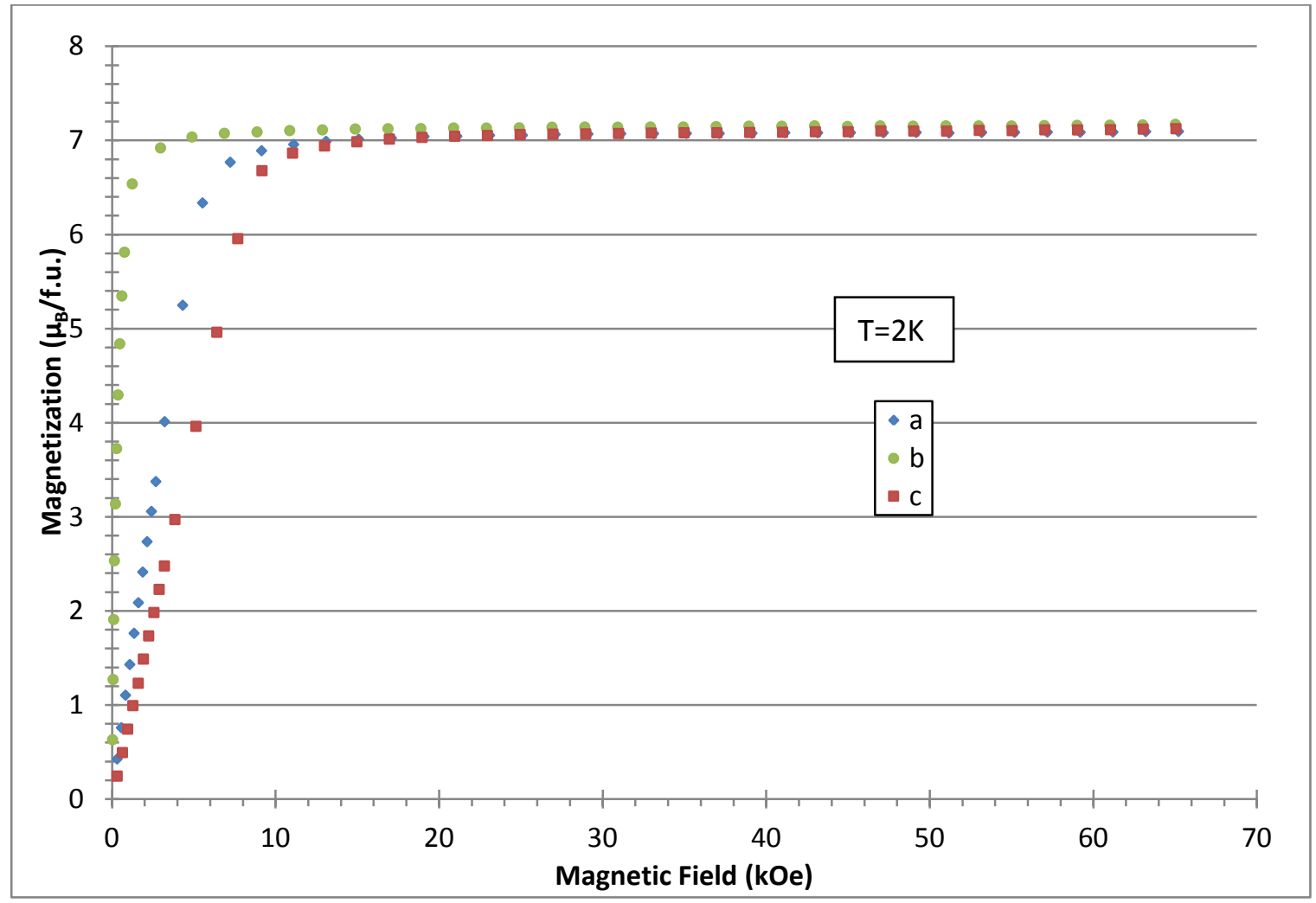

Figure 8.21: The isothermal magnetization of single crystal $\mathrm{GdNi}$ along $a, b$, and $c$-axes at $\mathrm{T}=2 \mathrm{~K}$. 


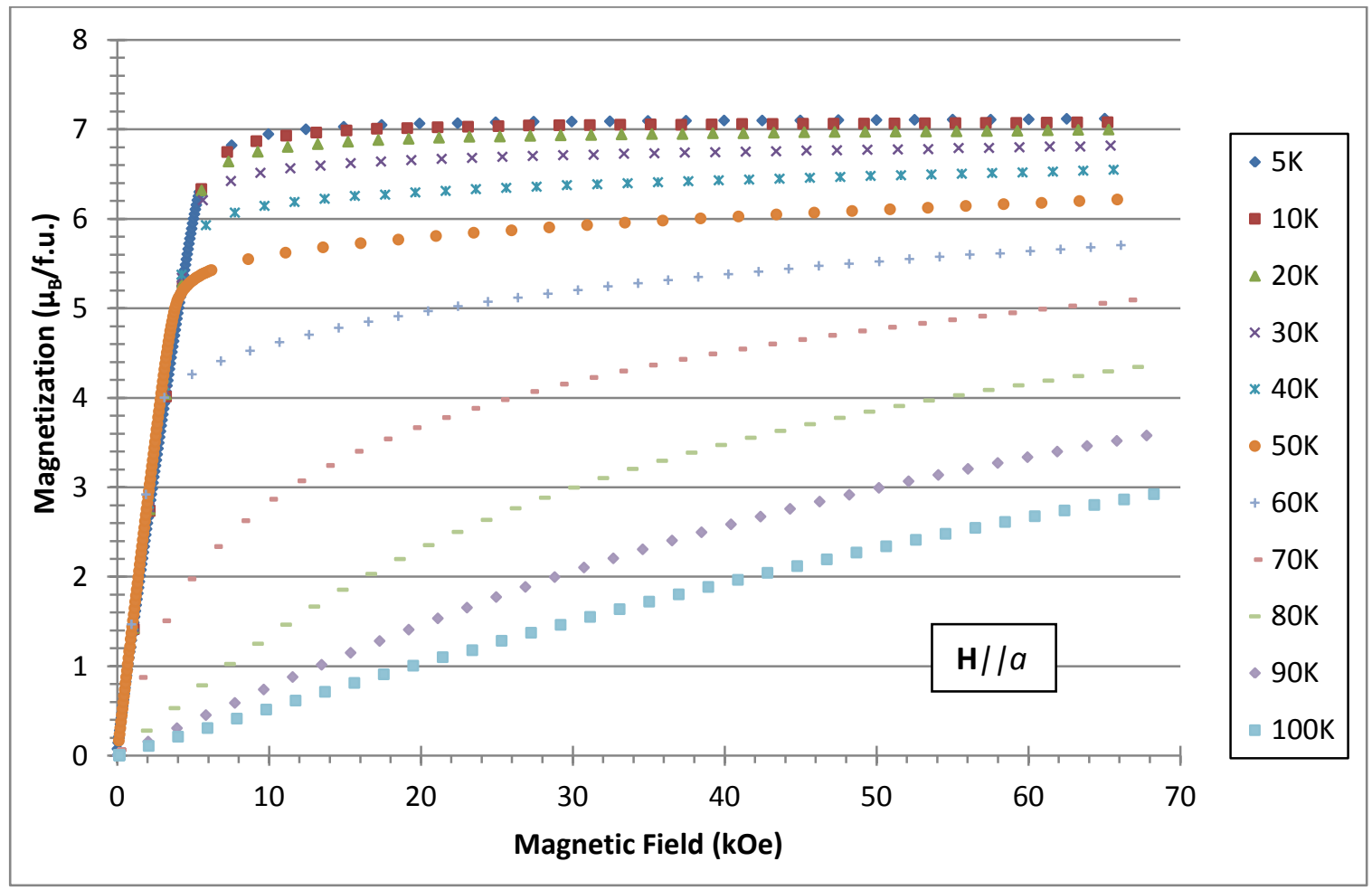

Figure 8.22: The isothermal magnetizations of single crystal GdNi from $T=5 \mathrm{~K}$ to $\mathrm{T}=100 \mathrm{~K}, \mathrm{H} \| \boldsymbol{a}$.

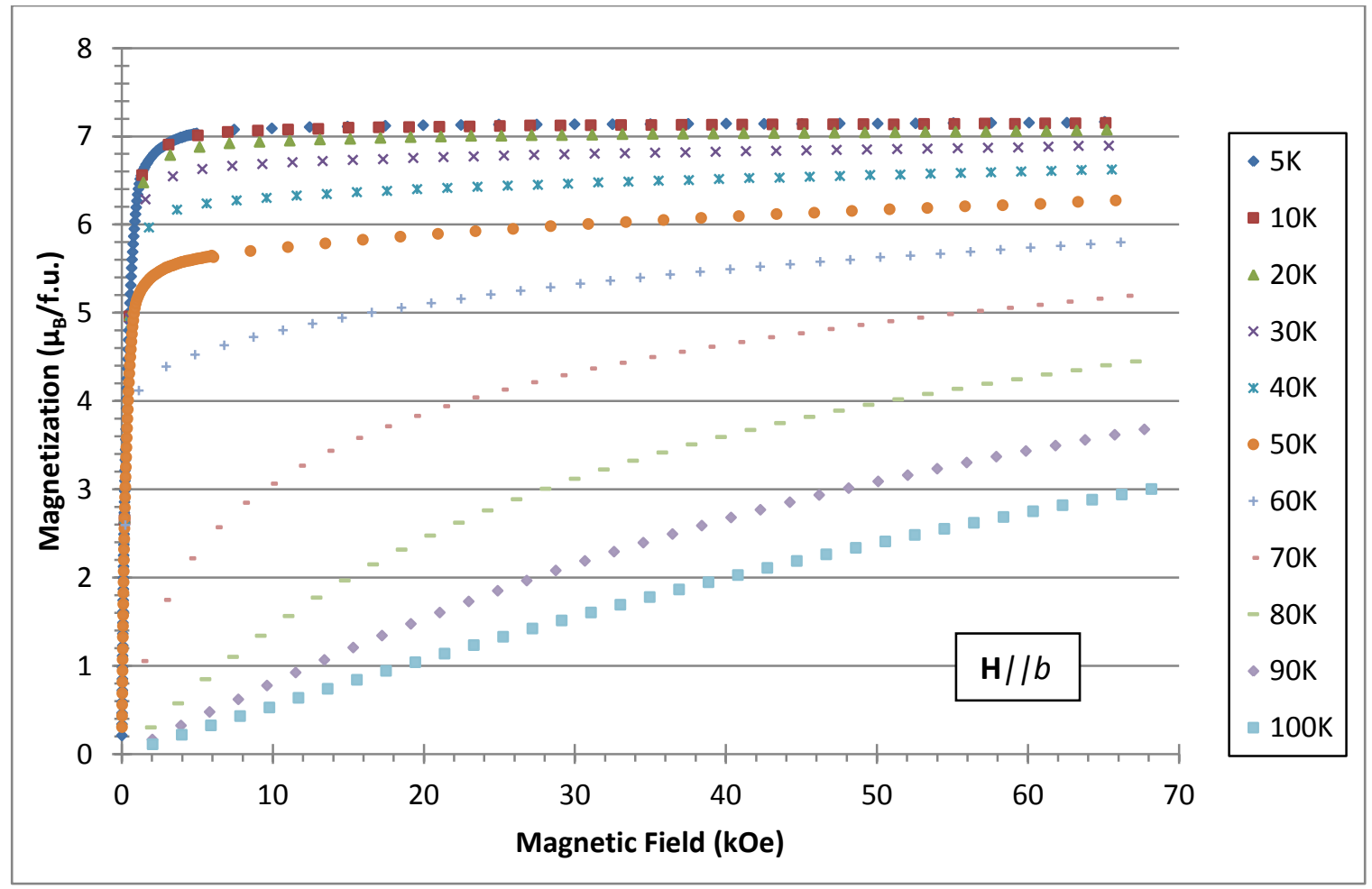

Figure 8.23: The isothermal magnetizations of single crystal GdNi from $T=5 K$ to $T=100 K, H \| b$. 


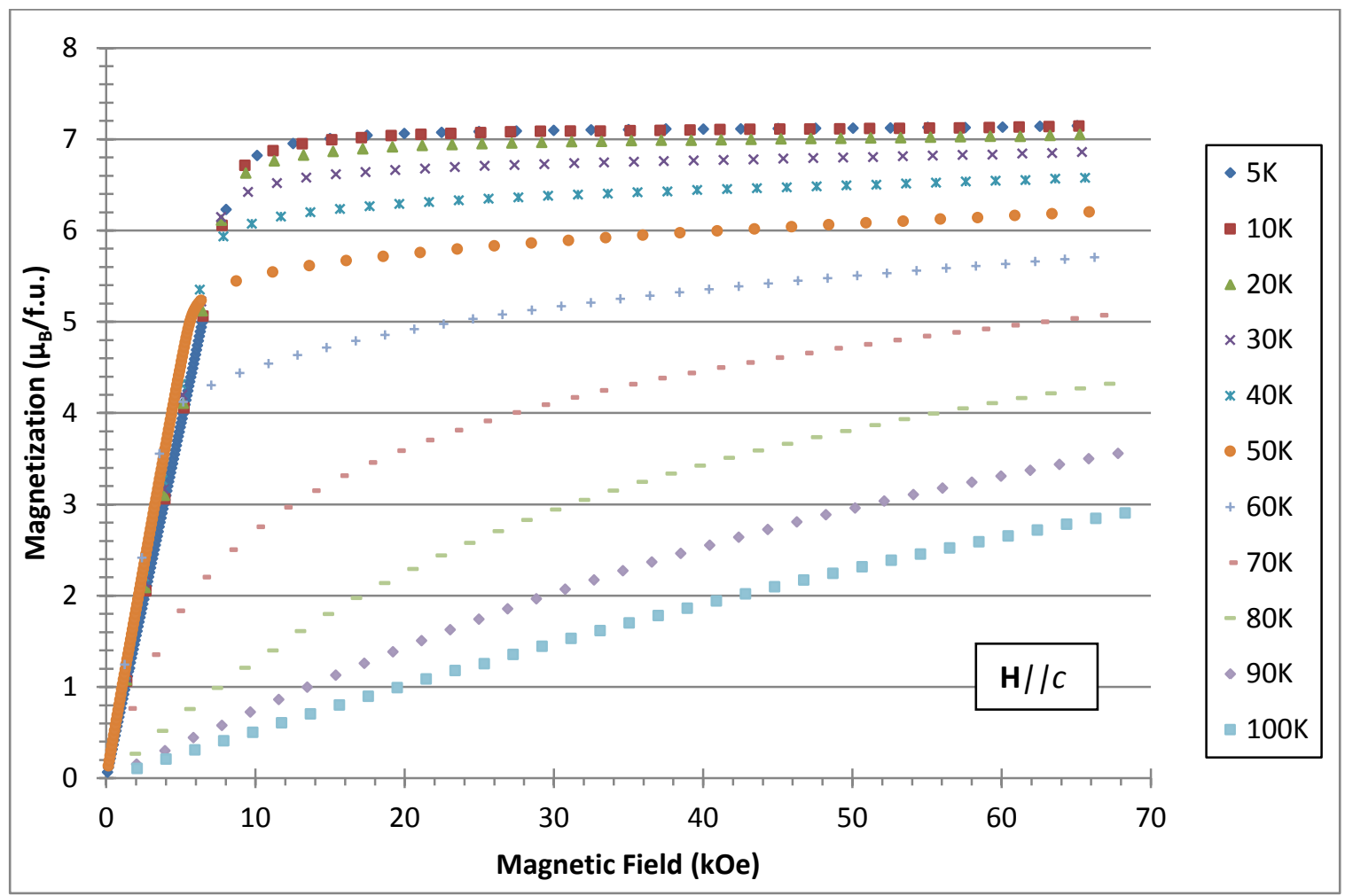

Figure 8.24: The isothermal magnetizations of single crystal $\mathrm{GdNi}$ from $\mathrm{T}=5 \mathrm{~K}$ to $\mathrm{T}=100 \mathrm{~K}, \mathrm{H} \| c$. 


\section{Chapter 9 Transport properties of GdNi}

The magnetoresistance of GdNi was measured at 10kOe. The resistivity curves are seen in Figure 9.1 and the calculated magnetoresistance is in Figure 9.2. In a 10kOe field, the magnetoresistance of $\mathrm{GdNi}$ is largest at $68 \mathrm{~K}$ which is expected with a $\mathrm{T}_{\mathrm{C}}$, of $69 \mathrm{~K}$. The peak magnetoresistance has a value of $-5.17 \%$. The magnetoresistance acts as predicted in theory, with a slightly larger resistance in field in the PM region and a drop in resistance at the magnetic ordering temperature.

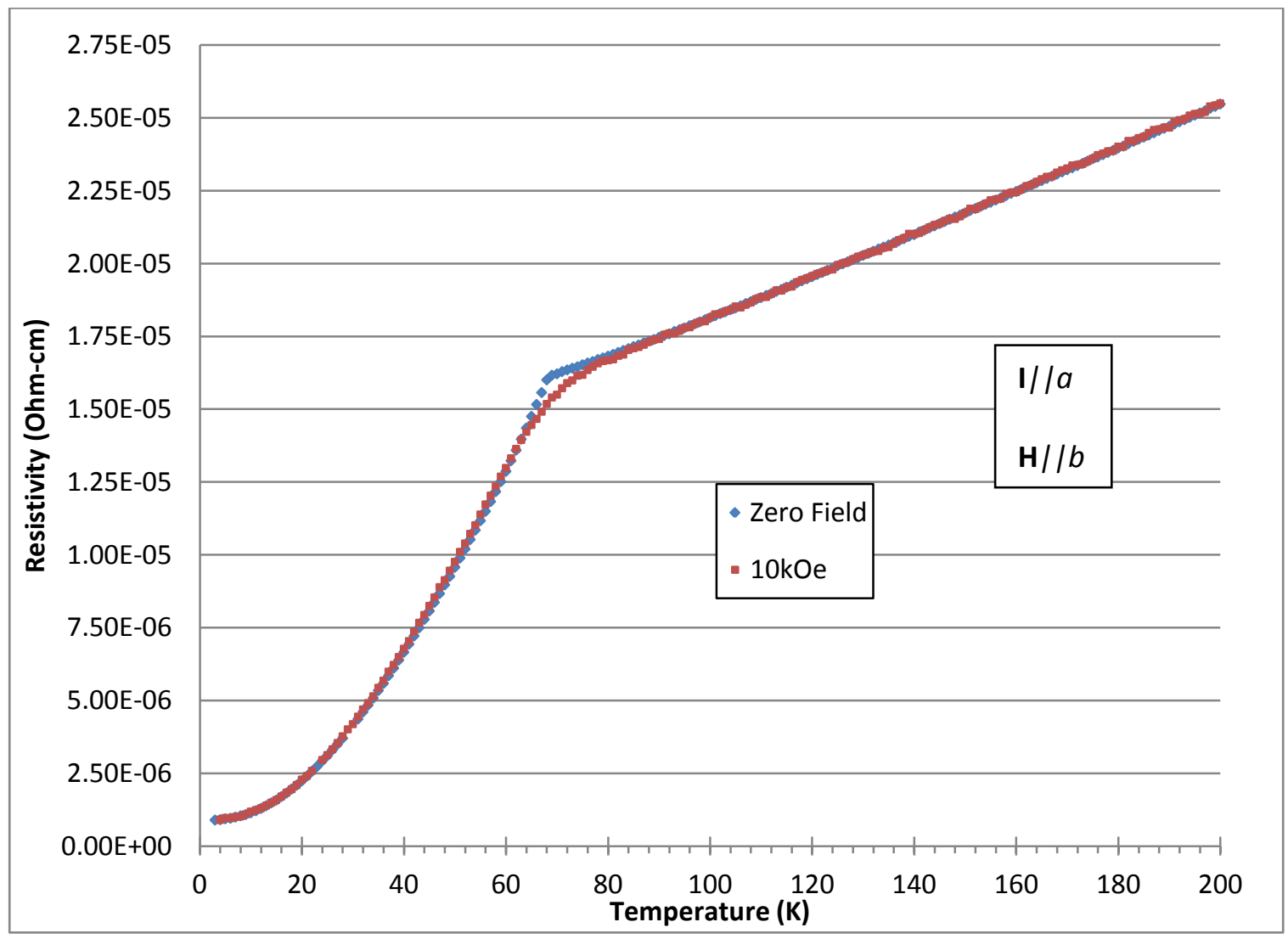

Figure 9.1: The resistivity vs. temperature curves for single crystal GdNi in zero field and 10kOe field, current flows parallel to the $a$-axis. Near $T_{C}$, the resistivity changes regimes, in a field the transition is rounded and the resistivity is slightly reduced. 


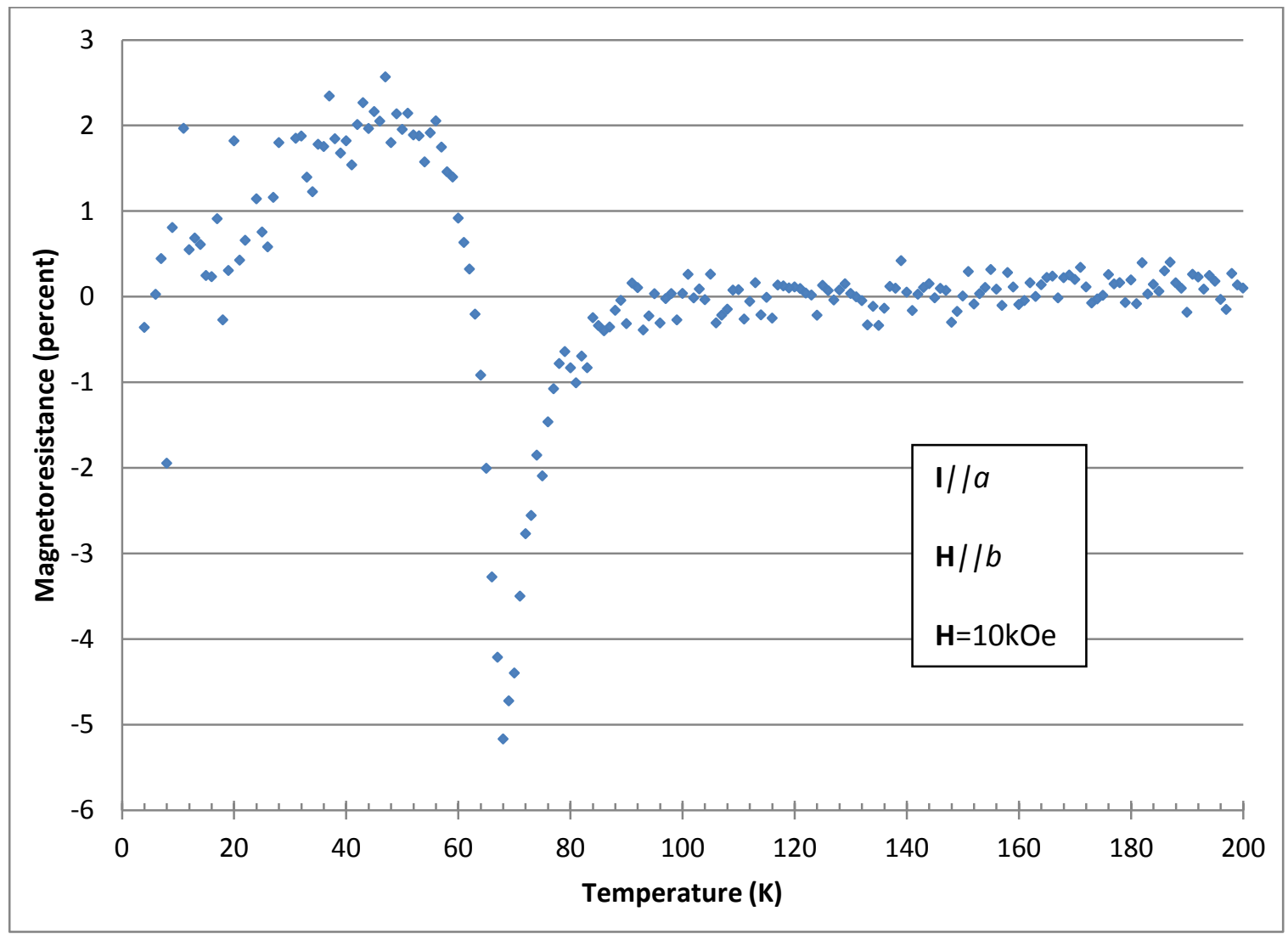

Figure 9.2: The magnetoresistance of single crystal GdNi, in a $10 \mathrm{kOe}$ field, current along the $a$-axis. Well above $T_{C}$ the magnetoresistance is barely positive. At $T_{C}$, magnetoresistance drops sharply, and below $T_{C}$ return to a positive value. 


\section{Chapter 10 Thermal properties of GdNi}

The heat capacity of GdNi was measured from about $2 \mathrm{~K}$ to $150 \mathrm{~K}$, and in zero field and 10,20 , and $50 \mathrm{kOe}$ magnetic fields along the easy magnetization $b$-axis. The brittle and flaky nature of GdNi made measurements in other directions challenging because samples fractured during preparation and became too small to use. The heat capacity of GdNi is seen in Figure 10.1. At $68 \mathrm{~K}$ a lambda-type transition is seen in the zero-field heat capacity data. This is in good agreement with a $T_{C}$ of $69 \mathrm{~K}$. In a magnetic field, the heat capacity peaks become progressively more rounded and shift to higher temperatures in increasingly stronger fields. The shift in the transition is as predicted for a FM to PM transition.

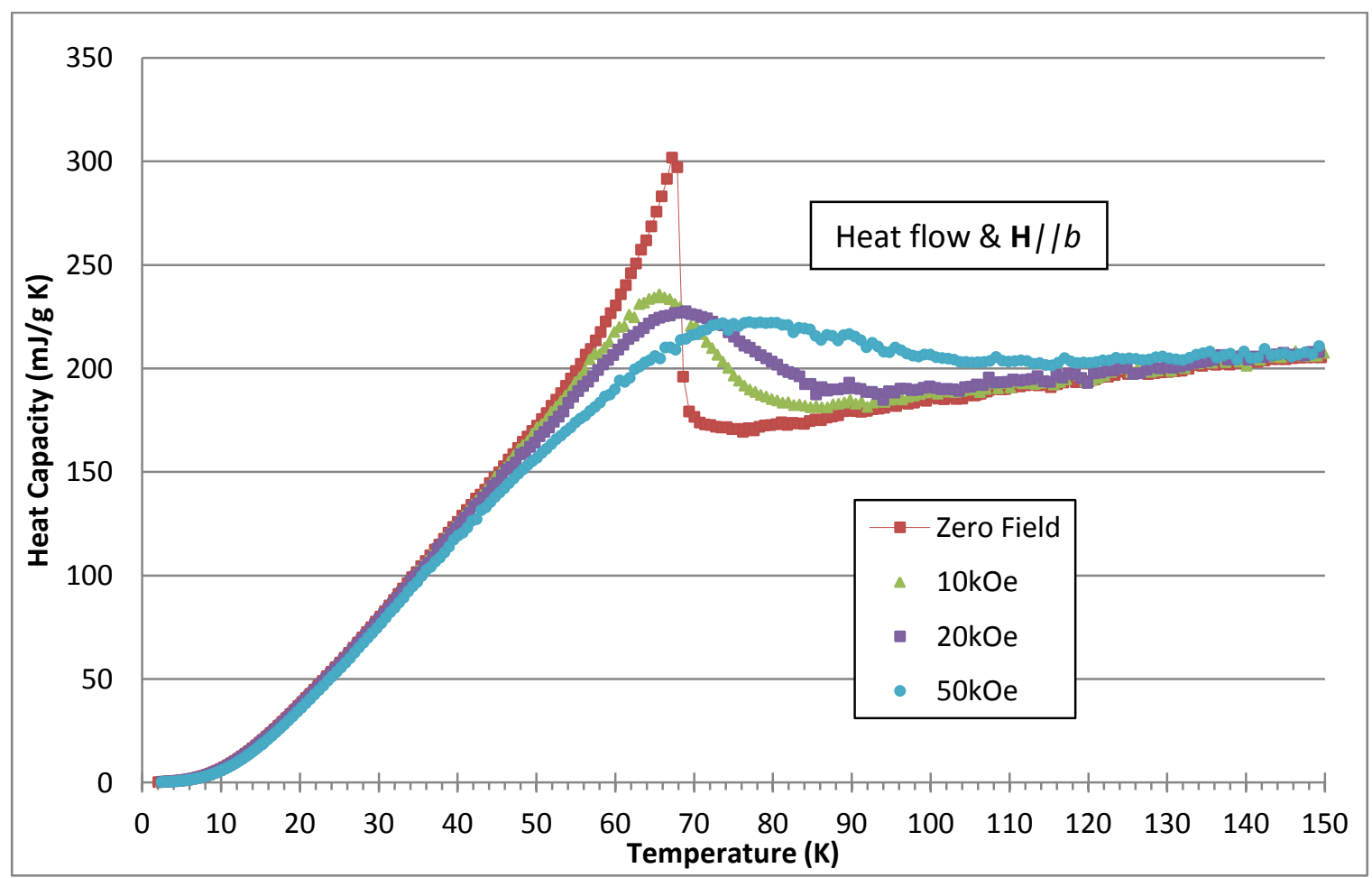

Figure 10.1: The heat capacity of a GdNi single crystal measured on the b-axis (easy magnetization direction) measured in zero field, and 10, 20, and $50 \mathrm{kOe}$ fields. A line is added to the zero field data to guide the eye. 
Through integration of the heat capacity curves, the entropy is calculated, and shown in Figure 10.2 . Only zero field and $50 \mathrm{kOe}$ data is shown to clearly portray the effect. Using equations (7) and (8), the adiabatic temperature change and the isothermal magnetic entropy change were calculated and shown in Figures 10.3 and 10.4. GdNi has a second order magnetic transition at the Curie temperature, seen as a broad peak in the adiabatic temperature change and the isothermal magnetic entropy change curves. Table 10.1 lists the $\Delta \mathrm{T}_{\mathrm{ad}}{ }^{\max }$ and $-\Delta \mathrm{S}_{\mathrm{M}}{ }^{\max }$ values from Figures 10.3 and 10.4, and the relative cooling power (RCP) calculated from the isothermal magnetic entropy change as $-\Delta \mathrm{S}_{\mathrm{M}}{ }^{\max }$ value multiplied by the full width half max of the peak. These values have previously been measured on polycrystalline $\mathrm{GdNi}^{19}$ and the single crystal $\mathrm{GdNi}$ data is measured to be about $10 \%$ better except for RCP values which are about $10-20 \%$ smaller.

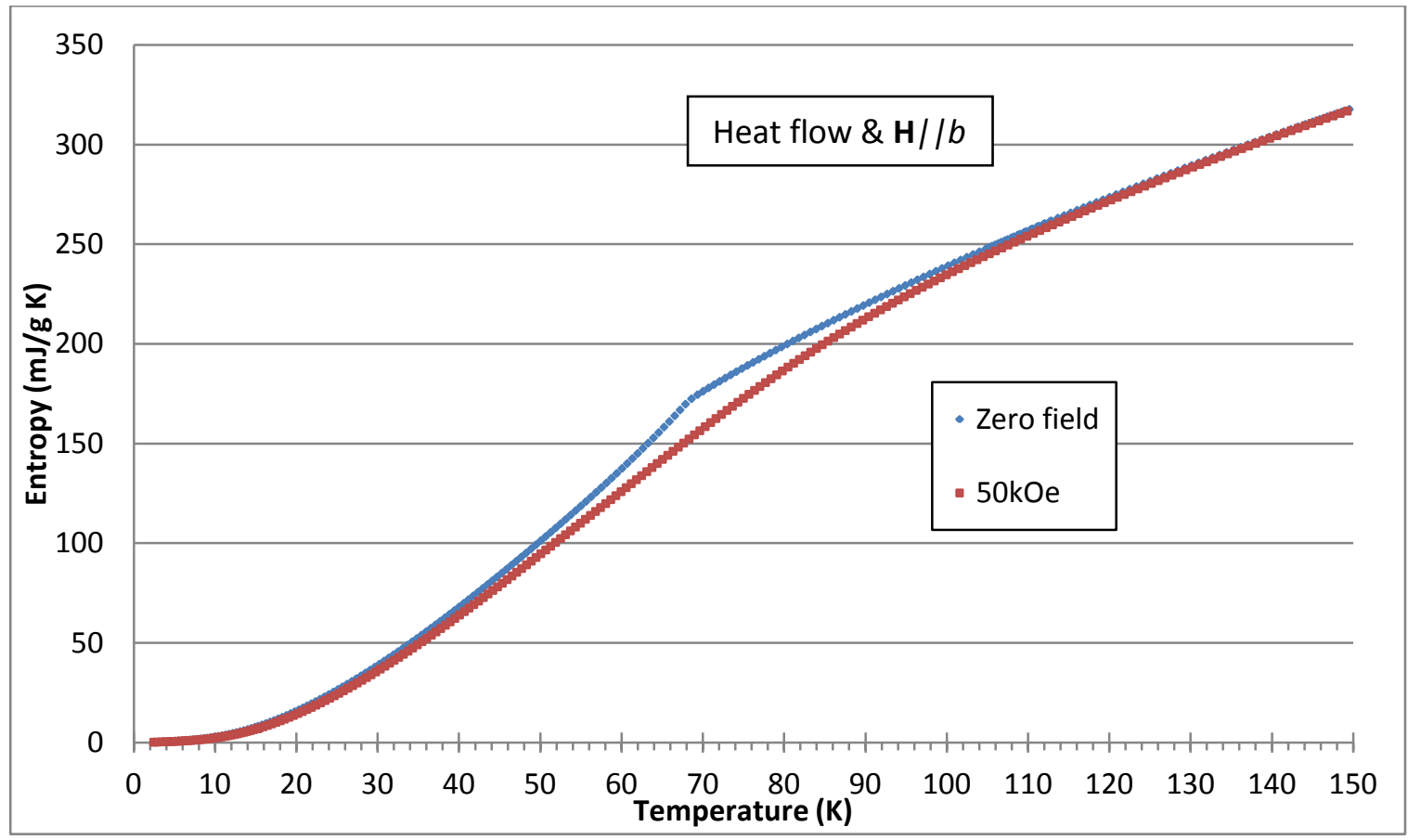

Figure 10.2: The temperature dependent entropy of single crystal GdNi. Only entropies in zero field and 50kOe are shown for clarity. In a magnetic field, the entropy is reduced near $T_{C}$. 


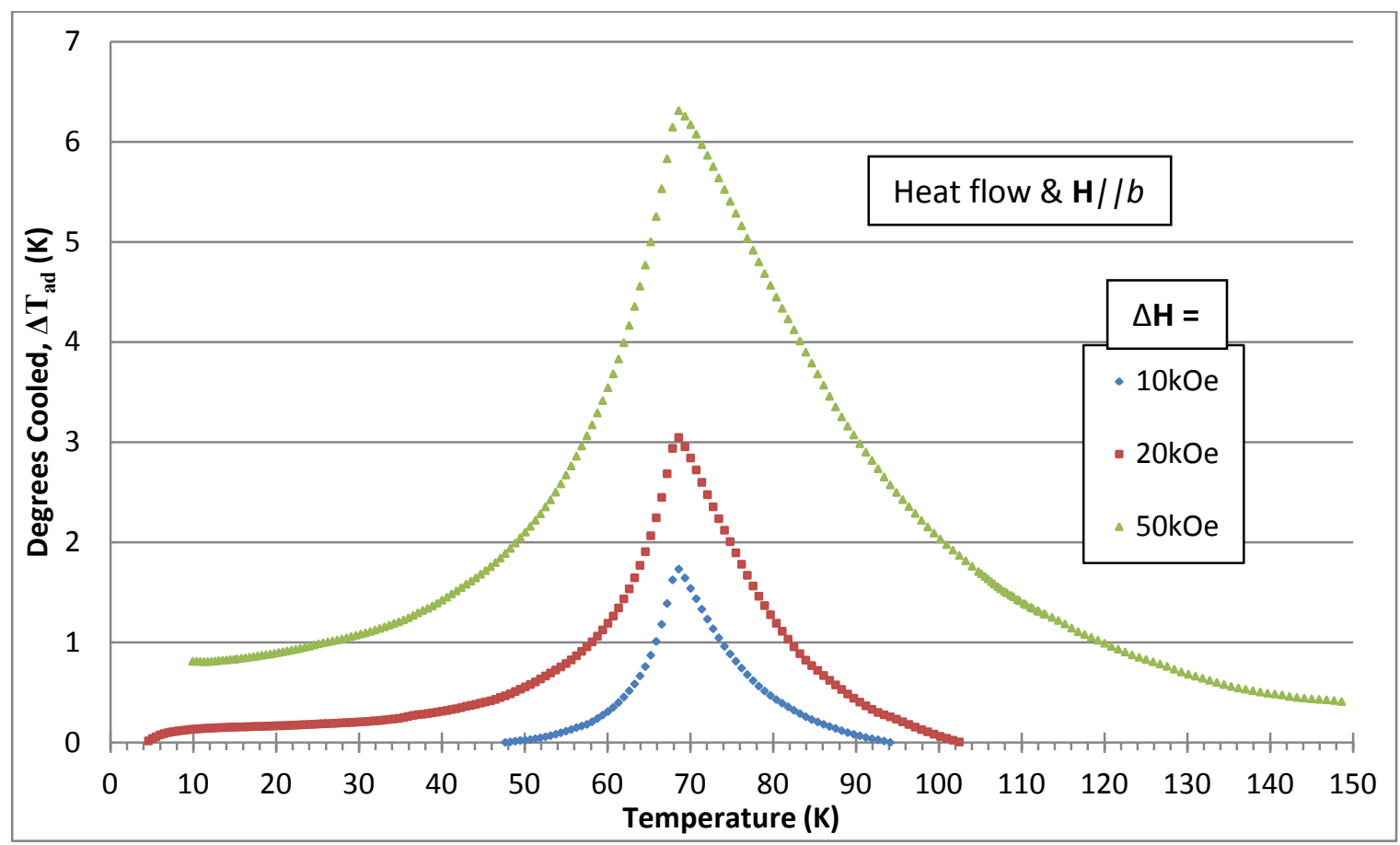

Figure 10.3: Adiabatic temperature change as a function of temperature in single crystal GdNi along the $b$-axis, calculated from temperature change due to a magnetic field applied from zero field to 10 , 20 and $50 \mathrm{kOe}$

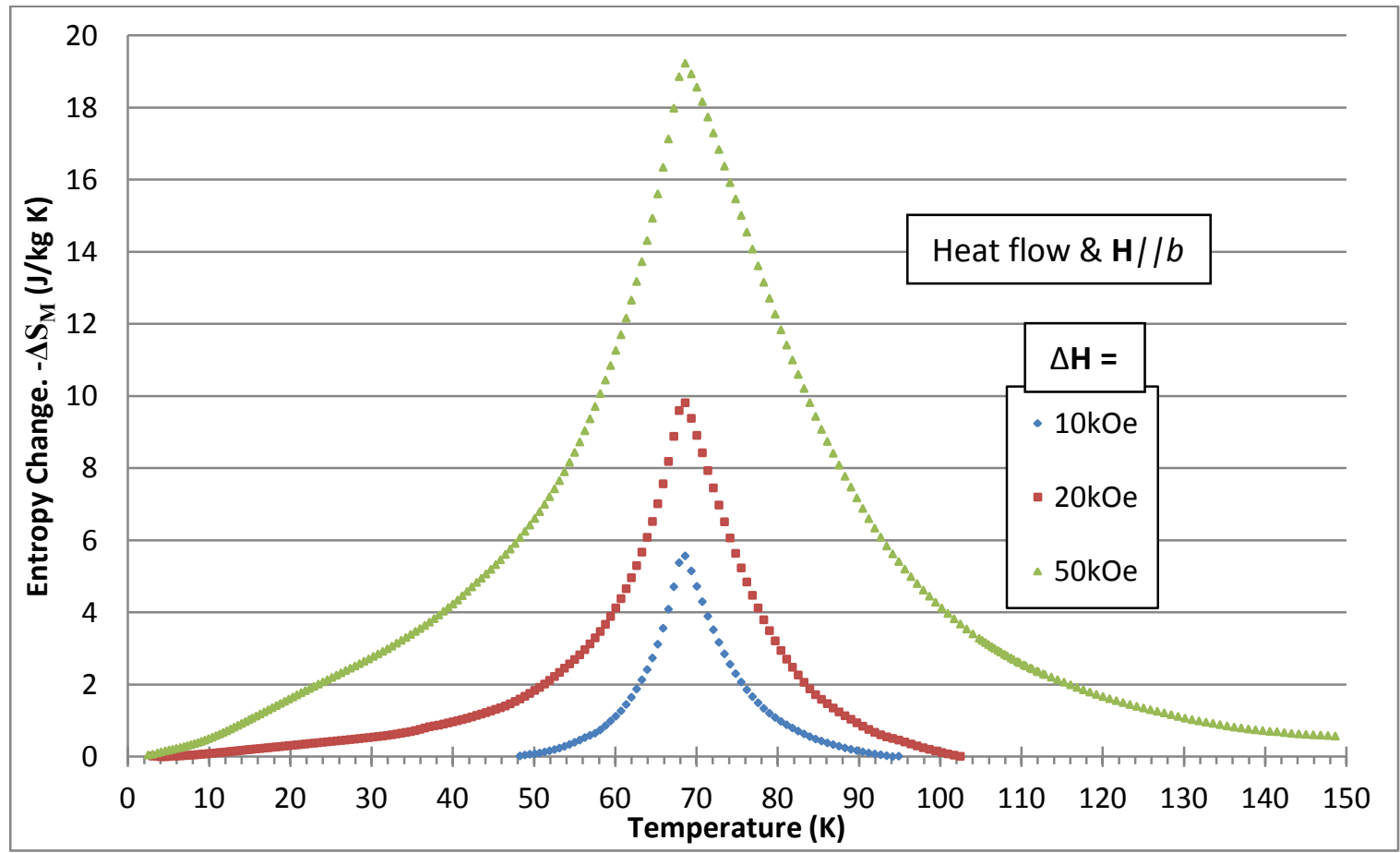

Figure 10.4: Isothermal magnetic entropy change plotted vs. temperature in single crystal GdNi along the $b$-axis, calculated from the entropy change due to a magnetic field applied from zero field to 10,20 and $50 \mathrm{kOe}$. 
Table 10.1: $\Delta T_{\mathrm{ad}}{ }^{\mathrm{max}},-\Delta \mathrm{S}_{\mathrm{M}}{ }^{\mathrm{max}}$ and $\mathrm{RCP}$ values calculated from the heat capacity measurements of GdNi

\begin{tabular}{|c|c|c|c|}
\hline$\Delta \mathrm{H}$ & $\begin{array}{c}\Delta \mathrm{T}_{\mathrm{ad}}^{\max } \\
(\mathrm{K})\end{array}$ & $\begin{array}{c}-\Delta \mathrm{S}_{\mathrm{M}}{ }^{\max } \\
(\mathrm{J} / \mathrm{kg} \mathrm{K})\end{array}$ & $\begin{array}{c}\mathrm{RCP} \\
(\mathrm{J} / \mathrm{kg})\end{array}$ \\
\hline $10 \mathrm{kOe}$ & 1.73 & 5.57 & 496 \\
\hline $20 \mathrm{kOe}$ & 3.05 & 9.82 & 140 \\
\hline $50 \mathrm{kOe}$ & 6.31 & 19.2 & 49 \\
\hline
\end{tabular}




\section{Chapter 11 Conclusions}

Several methods of GdNi single crystal growth in specially fabricated crucibles were developed and refined. Tantalum boride coated tantalum and W-backed $\mathrm{Gd}_{2} \mathrm{O}_{3}$ crucibles both produced single crystals suitable for the characterization measurements. The $\mathrm{Gd}_{2} \mathrm{O}_{3}$ crucible was found to produce larger crystals with fewer impurities and is currently best crucible and best method for GdNi single crystal growth. In the process of refining crucible methods, the newly developed crucibles can be applied to other compounds and future research, opening research paths for materials previously thought to be too reactive for single crystal processing.

Characterizations were performed on single crystal GdNi, measuring DC magnetization, AC susceptibility, magneto-resistivity, and magneto-thermal properties. Many of the measured properties were improvements over those that had been previously measured. Heat capacity measurements are the first measurements reported on a single crystal. From the DC magnetization measurements, the Weiss temperature $\left(\theta_{\mathrm{p}}\right)$ was calculated as $72.1 \pm 0.4,72.4 \pm 0.4$, and $71.7 \pm 0.5 \mathrm{~K}$ for the $a$ - $b$ - and $c$-axes, respectively, and effective moment ( $\mathrm{p}_{\text {eff }}$ ) was found to be $8.212 \pm 0.014,8.278 \pm 0.014$, and $8.162 \pm 0.014 \mu_{\mathrm{B}} /$ f.u., for the same respective axes. The $\mathrm{Gd}^{3+}$ magnetic ion has an effective moment of $\mathrm{p}_{\text {eff }}=7.94 \mu_{\mathrm{B}}$ and there is additional contribution from conduction electrons. The saturation magnetization was measured to be $7.10 \pm 0.03 \mu_{\mathrm{B}} /$ f.u. along the $a$-axis, $7.17 \pm 0.03 \mu_{\mathrm{B}} /$ f.u. along the $b$-axis, and $7.12 \pm 0.03 \mu_{\mathrm{B}} /$ f.u. along the $c$-axis, all slightly higher than $7 \mu_{\mathrm{B}}$ from the $\mathrm{Gd}^{3+}$ magnetic ion from $\mathrm{Gd} 5 d$ and Ni $3 d$ contributions. The $\Delta \mathrm{T}_{\mathrm{ad}}{ }^{\max }$ and $-\Delta \mathrm{S}_{\mathrm{M}}{ }^{\max }$ values were measured on a single crystal along the $b$-axis in differential fields of 10,20 , and $50 \mathrm{kOe}$. For each of the differential fields, $\Delta \mathrm{T}_{\mathrm{ad}}{ }^{\max }$ was $1.73,3.05$, and $6.31 \mathrm{~K}$ and - 
$\Delta \mathrm{S}_{\mathrm{M}}{ }^{\max }$ was $5.57,9.82$ and $19.2 \mathrm{~J} / \mathrm{kg} \mathrm{K}$. These values were found to be slightly larger than values previously reported. Kumar et al. calculated $-\Delta S_{M}{ }^{\max }$ values of 9 and $17 \mathrm{~J} / \mathrm{kg} \mathrm{K}$ in 20 and $50 \mathrm{kOe}$ fields measured on polycrystalline $\mathrm{GdNi}^{19}$

The data collected on single crystalline GdNi is solid and conclusive. Few questions remain about GdNi from the current perspective. The future of GdNi research is likely to be found in the $\mathrm{R}_{\mathrm{x}} \mathrm{R}{ }_{1-\mathrm{x}} \mathrm{Ni}$ system where rare earth substitutions will provide new processing challenges and new parameters to tailor the unique magnetic properties of the GdNi system. 


\section{References}

${ }^{1}$ V. F. Novy, R.C.Vickery, E. V. Kleber, Trans. Met. Soc. AIME 221, 585 (1961).

${ }^{2}$ N.C. Baezinger and J.L. Moriarty, Jr., Acta Cryst. 14, 946 (1961).

${ }^{3}$ R.E. Walline and W.E. Wallace, J. Chem. Phys. 41, 1587 (1964).

${ }^{4}$ S.C. Abrahams, J. L. Bernstein, R. C. Sherwood, J. H. Wernick, and H. J. Williams, J. Phys. Chem. Solids 25, 1069 (1964).

${ }^{5}$ I. Ursu and E. Burzo, J. Magn. Reson. 8, 274 (1972).

${ }^{6}$ K. Sato, Trans. JIM 21, 336 (1980).

${ }^{7}$ K. Sato, K. Koyachi, K. Mori, J. Appl. Phys. 52, 2084 (1981).

${ }^{8}$ Y. Isikawa, K. Higashi, T. Miyazaki, and K. Sato, J. Magn. Magn. Mater. 31-34, 1057 (1983).

${ }^{9}$ K. Sato, Y. Isikawa, K. Mori, and T. Miyazaki, J. Appl. Phys. 67, 5300 (1990).

${ }^{10}$ J. A. Blanco, J. C. Gómez Sal, J. Rogriguez Fernandez, D. Gignoux, D. Schmitt, and J.

Rodriguez-Carvajal, J. Phys.: Condens. Matter 4, 8233 (1992).

${ }^{11}$ P. L. Paulose, S. Patil, R. Mallik, E. V. Sampathkumaran, and V.

Nagarajan, Physica B 223-224, 382 (1996).

${ }^{12}$ R. Mallik, P. L. Paulose, E. V. Sampathkumaran, and V. Nagarajan, Phys. Rev. B 55, 8650 (1997).

${ }^{13}$ V. L. B. de Jesus, V. M. T. S. Barthem, I. S. Oliveira, and A. P. Guimarães, J. Magn. Magn. Mater. 177-181, 1125 (1998).

${ }^{14}$ K. Yano, I. Umehara, K. Sato, and A. Yaresko, Solid State Commun. 136, 67 (2005).

${ }^{15}$ E. Gratz and A. Lindbaum, J. Magn. Magn. Mater. 177, 1077 (1998). 
${ }^{16}$ K. Uhlířová, J. Prokleška, J. Poltierová Vejpravová, V. Sechovský, and K. Maezawa, J. Magn. Magn. Mater. 310, 1753 (2007).

${ }^{17}$ D. Paudyal, Ya. Mudryk, Y. B. Lee, V. K. Pecharsky, K. A. Gschneidner, Jr., and B. N. Harmon, Phys. Rev. B 78, 184436 (2008).

${ }^{18}$ N. V. Baranov, H. Michor, G. Hilscher, A. Proshkin, and A.Podlesnyak, J. Phys.:

Condens. Matter 20, 325233 (2008).

${ }^{19}$ P. Kumar, K. G. Suresh, A. K. Nigam, and O. Gutfleisch, J. Phys. D: Appl. Phys. 41, 245006 (2008).

${ }^{20}$ P. Villars and L.D. Calvert, Pearson's Handbook of Crystallographic Data for Intermetallic Compounds, (second ed.), ASM International, Materials Park, OH (1991).

${ }^{21}$ N. H. Duc, in Handbook on the Physics and Chemistry of Rare Earths, edited by K. A. Gschneidner, Jr. and L.R. Eyring (North-Holland Physics Publishing, Amsterdam, 1984), Vol. 24, Chap. 48, p. 339.

${ }^{22}$ E. Gratz and A. Lindbaum, J. Magn. Mater. Mater. 137, 115 (1994).

${ }^{23}$ J. Joule, Sturgeon's Annals of Electricity 8: 21 (1842).

${ }^{24}$ V. K. Pecharsky and K. A. Gschneidner, Jr., Pure Appl. Chem.79, 1383 (2007).

${ }^{25}$ D. Paudyal, V. K. Pecharsky, K. A. Gschneidner, Jr., and B. N. Harmon, Phys. Rev. B 75, 094427 (2007).

${ }^{26}$ V. K. Pecharsky, G. D. Samolyuk, V. P. Antropov, A. O. Pecharsky, and K. A.

Gschneidner, Jr., J. Solid State Chem. 171, 57 (2003).

${ }^{27}$ E. Warburg, Ann. Phys. Chem., 13, 141 (1881).

${ }^{28}$ K. Klepp and E. Parthé, Acta Cryst. B36, 774 (1980).

${ }^{29}$ K. Klepp and E. Parthé, J. Less-Common Met.85, 181 (1982). 
${ }^{30}$ E. Parthé, in Crystal Structures and Crystal Chemistry of Ternary Rare EarthTransition Metal Borides, Silicides and Homologues, in: Handbook on the Physics and Chemistry of Rare Earths, edited by K. A. Gschneidner, Jr. and L.R. Eyring (NorthHolland Physics Publishing, Amsterdam, 1984), Vol. 6, Chap. 48, p.113-334.

${ }^{31}$ Materials Preparation Center, Ames Laboratory of U.S. DOE, Ames, IA, USA www.mpc.ameslab.gov.

${ }^{32}$ V. K. Pecharsky, J. O. Moorman, and K. A. Gschneidner, Jr., Rev. Sci. Instrum. 68, 4196 (1997).

${ }^{33}$ T.B. Massalski, H. Okamoto, P.R. Subramanian and L. Kacprzak, (second ed.), ASM International, Materials Park, OH (1990).

${ }^{34}$ N. V. Tristan, S. A. Nikitin, T. Palewski, K. Skokov, J. Warchulska, J. Alloys Comp. 334, 40 (2002).

${ }^{35}$ R. Mallik, P. L. Paulose, E. V. Sampathkumaran, S. Patil, and V. Nagarajan, Phys. Rev. B 55, 8369 (1997).

${ }^{36}$ K. Nishimura, K. Mori, Y. Narita, and W. D. Hutchison, J. Magn. Magn. Mater. 310, 1730 (2007).

${ }^{37}$ K. A. Gschneidner, Jr , and F. A. Schmidt, Determination of the Optimum Processing Parameters for the Preparationof Carbide Coated Refractory Metal Objects. Internal Communication (2008).

${ }^{38}$ D. Paccard, J. Allemand, J.M. Moreau, N. Marcano, J.C. Gomez Sal, Solid State Sciences, 7, 776 (2005).

${ }^{39}$ A. E. Dwight, R. A. Conner, Jr., and J. W. Downey, Acta Cryst., 18, 837 (1965).

${ }^{40}$ D. Chen, E. Pardo, and A. Sanchez, IEEE Trans. Mag. 38, 1742 (2002). 